borno LES MOTIFS PRIMITIFS DU DESSIN DES AILES DES LEPIDOPTERES ET LEUR ORIGINE PHYLETIQUE

BOTKE, JACOB 1916

Published on demand by

UNIVERSITY MICROFILMS INTERNATIONAL Ann Arbor, Michigan, U.S.A. · London, England 






\section{ONDERZOEKINGEN}

verricht in het Zoölogisch Laboratorium der Rijksuniversiteit Groningen. 



\section{ONDERZOEKINGEN}

verricht in het Zoölogisch Laboratorium der Rijksuniversiteit Groningen.

$$
\text { V. }
$$

LES MOTIFS PRIMITIFS DU DESSIN DES AILES DES LÉPIDOPTĖRES ET LEUR ORIGINE PHYLÉTIQUE

\section{J. BOTKE,}

DOCTEUR Ès SCIENĆES.

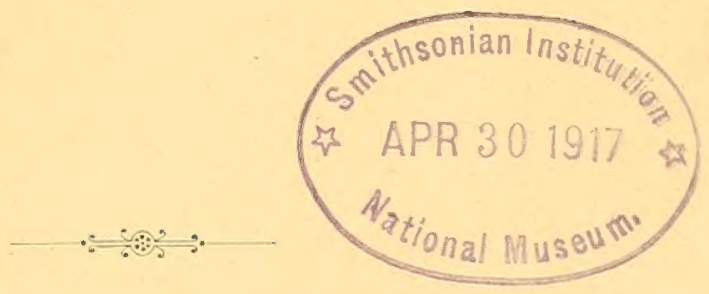

N. V. BOEKHANDEL EN DRUKKERIJ

voorheEN E. J. BRILL, LEIDEN IgI6. 



\title{
LES MOTIFS PRIMITIFS DU DESSIN DES AILES DES LÉPIDOPTĖRES ET LEUR ORIGINE PHYLÉTIQUE
}

PAR

\author{
J. BOTKE.
}

(Avec 4 planches et 12 figures dans le texte).

\section{CHAPITRE I.}

\section{INTRODUCTION.}

\section{A. Historique.}

Si l'on reut décourrir l'évolution phylogénétique d'une certaine espèce, d'un certain groupe d'animaux ou d'un organe donné, il faut se mettre au courant de tout ce que l'étude de l'ontogenèse de cette espèce, de ce groupe ou de eet organe a mis au jour; il faut avoir devant less yeux les faits morphologiques qui sont connus à l'égard de ce sujet; les aberrations individuelles et les anomalies, parce que celles-ci sont très souvent d'une nature atavique, mais il faut aussi tenir compte de tout ce qu'il nous est resté de leurs représentants éteints dans les fossiles.

Enfin les expériences peuvent être de grande valeur.

\section{Les recherches ontogénétiques.}

Pour la connaissance de la phylogenèse du dessin des ailes, l'ontogenèse semble n'avoir aucune importance. Nous disons "semble", car, quoique ce que DrXey (14) a dit en 1890 ne soit pas tout à fait inexact:

"It must not be forgotten that the investigation is deprived of the assistance, usually afforded to similar enquiries from the side of embryology, for the characters in question spring as it were 
at once into perfect being at a definite stage in the life of the individuae; they have, so to speak, no growth, no embryology", cette ,assistance" n'est pas entièrement absente et elle a rendu en réalite d'importants services aux investigations phylogénétiques.

Mais les ailes des insectes holométaboliques n'apparaissent pas si subitement que Drxey se l'imagine, la chrysalide aussi. les posside déji et Vax Bemmesex (1 et 2) a démontré que le dessin des ailes imaginales parcourt bien avant l'éclosion du papillon un déveloplement of montre des stades dont la commaisinae importe beaucoup à l'histoire phylétique.

En ouvrant la peau de la chrysalide à des époques différentes entre la formation de la chrysalide et l'éclosion du papillon, il vit paraître sur les ailes de Pyrameis cardui et de Vanessa urticae quelques jours apres la transformation de la chenille en chrysalide, aussitôt que le développement des écailles avait commencé, un dessin, différant beatucoup de celui de l'insecte adulte, mais montrant néanmoins quelques points de rapport.

Quant à Pyrameis cardui, quatre jours après la formation de la chrysalide, la couleur de fond des ailes antirieures etait d'un jaune-brun clair cot celle des ailes postérieures d'un brun un peu plus foncé; ('n mime temps les dernieres citaient plus translucides que les premières.

Dans les cellules Ic, II, III, IV, V, VI et VII + VIII des ailes antérieures, il y avait des taches plus claires, qui étaient cernées du côté de la partie basilaire de l'aile par des taches plus foncées en forme de fancille. Tiendroit où la nervure de la cellule médiane devait se développer, par conséquent la partie latérale de la cellule médiane, était aussi plus clair que l'autre partie de l'aile.

Or ce dessin ne se modifie pendant le développement ultérieur que d'une façon très lente, et très légèrement. La couleur de fond sassombrit d'abord, d'une facon continue, "nsuite clle seclaireit. Les taches dans le's cellules marginales s'échireissent et se dessinent plus nettement. Dans la cellule $I b$ on voit paraître une tache retardée. L'area claire dans la partie latérale de la cellule médiane 
disparaît bientôt et est remplacée par une bande, composée de trois taches au milieu du bord antérieur et cela pendant les derniers jours avant que le dessin définitif apparaisse. Lianteur a vu paraîtro le dessin définitif trente-six heures avant que le papillom s'envolât, de la même manière qu'une photographie se développe peu à peu dans le bain. Le dessin antérieur, restant done le même pendant toute la vie chrysalidaire avec de petits changements sculement, est effacé partiollement par lo dessin ultérieur, tandis qu'une autre partie va participer à sa composition, comme si rein neues Gemälde über eine alte halb verwischte und verblichene Decoration hingemalt würde".

Les taches longeant le bord externe se maintiennent, les antérieures du moins. Les taches postérieures s'assombrissent de plus en plus et se perdent enfin dans le noir et le rouge du dessin de l'adulte; les autres augmentent en clartí, deviomnent d'un blan" très pur et prennent des dimensions différentes, tandis qu'au commencement elles se ressemblaient assez, quant à leur étendue. P'uis il a pu constater que les ailes anterieures ot postérieures a leurs surfaces supérieure et inférieure avaient le même dessin primaire.

Vanessa urticae parcourt un déreloppement semblable; ici encore le dessin primaire se montre bientôt après le commen"ement du stade chrysalidaire, tandis que la déoration définitive paraît également assez brusquement au terme de la période nymphale. De même la rangée de taches marginales se retrouve, quoiqu'elles ne soient pas si distinctes que chez Pyrameis carlui; dans le dessin définitif, au contraire, les deux taches antéricures seules se maintiennent, mais elles se fondent et forment une tache claire et plus grande comme cela était aussi le cas chez $P$. cardui.

Arant Van Beymelen des recherches semblables ont été déjà faites par Scnüffer (63) pour Tanessa urticae. Tandis que ce dernier pensait que la première ornementation des ailes portait dojia les caractires principaux du dessin complet ultérieur, desorte qu'on ne pouvait lui attribuer que peu de valeur, quant aux buts phylogénétiques, c'est le premier qui a acquis un tout autre résultat; il voit dans les premières figures le dessin du genre, ce 
qu'il y a de commun dans les dessins des différentes espèces de Tranesse ulterieurement souvent si divergents. De plus il constata que le dessin définitif se compose de quelques restes de l'ornementation primitive et d'un dessin nouveau, qui se montre peu de temps avant l'éclosion.

Il a été soutenu dans cette opinion par DrXer, qui indépendamment de l'étude de VAN Bemmenes et exclusivement par la comparaison des différentes adultes du mêmer genre, arriva à considérer la rangée de taches, dont nous avons parlé, comme la partie pliylogénétiquement la plus ancienne du dessin.

Nous pouvons ainsi distinguer avec VAN BEMMELEN dans le développenent ontogénétique des ailes au moins trois phases importantes; la premiere, la phese pumule, sur les ailes de la chrysalide [ou, comme on dit très souvent, mais à tort, selon nous, sur les "gaines ou étuis" des ailes (voir aussi DEEGENER 13, pag. 17)]; la seconde, la pluse intramupule, sur les ailes imaginales pendant le temps qu'elles sont encore enfermées dans la peau de la chrysalide, et la troisième, la phase imaginale, que nous pouvons observer chez l'insecte adulte. Nous traiterons d'abord de ce que les études de ces deux premiers stades nous ont appris et, après, du dessin imaginal dans le chapitre: "Les recherches morphologiques comparées".

Commençons par le dessin pupal et constatons tout de suite que le nombre des recherches touchant ce sujet est encore très restreint.

C'est que beaucoup de chrysalides ne s'y prêtent guère, parce que souvent peu après la dernière mue de la chenille, la peau chrysalidaire prend une conlenr brune ou hrun-noir, de sorte quil n'est plus question de dessin. Il n'y a que celles chez lesquelles ce brun uniforme ne se développe pas, qui puissent nous être utiles et qui en effet l'aient déjà été.

Poultor (60) avait indiqué déjà en 1891 une ligne ou un sillon sur les ailes chrysalidares de cortaines Vanessides qui lonere le bord extérieur, mais pas tout à fait parallèle à celui-ci et qui ne peut être autre chose que la délinéation du bord externe d'une 
aile qui se rapproche plus du stade imaginal que du stade pupal, mais sen distingue par unc moindre différenciation. Fn partant de ce point de rue, il donne done à la sculpture une signification phylogénétique.

Dans le même mémoire Poulton a démontré que l'aile de la chrysalide en son entier représente un stade ancestral, car chez certains Lépidoptères féminins, comme Fumea nitidella, Orgyia antiqua, Hybermia defoliaria etc. qui ont perdu leurs ailes et ou des ruliments rappellent seulement une époque ancestrale disparur", il trouve dans les chrysalides qui vont donner naissance à des femelles aptiores, des ailes plus dévelopheres qui, quoique bien plus courtes que celles des chrysalides mâles, ont pourtant dẹ dimensions considérables quand on les compare avec les rudiments extrêmement petits des adultes femelles.

C'est cependant VAN Bemanen (5) qui pour la première fois examina plus exactement un certain nombre de chrysalides en vue du dessin des ailes.

Quelques Vanessides (Vunessa urticae, V. io, Pyrameis atalanta, $P$. cardui et Araschmin prorsa), un certain nombre de Papilionides (Pupilio machaon, P. podalivins, Thais polyxena ete.) et les Piérides suivantes: Pieris brassicae, $P$. nupi, Gonepteryx rhumi, Aporia cratuegi et Euchloë cardamines, étaient les sujets de ses recherches et Iui firent conclure que l'ornementation chrysalidaire montre un plau commun qui pourrait être utile au jugement des relations d'affinité entre les ordres différents.

Ce plan se compose, selon l'auteur, d'une extension de pigment, partiollement le long des nervures, partiellement longitudinalement sur la bissectrice des espaces internervuraux.

Chez Aporia crataegi ce pigment internervural s'amasse en forme de taches.

En outre il aperçut chez les chrysalides examinées deux rangées transversales de taches internervurales plus claires; l'une longe le bord externe de l'aile, l'autre, plus intérieure, se trouve à peu pres au milieu de la première et de la seconde nervure transversale discoïdale. 
Ces taches ne sont pas toujours de la même clarté, mais montrent toutes sortes de stades de régression jusqu'à l'absence totale. De l'autre côté, elles se sont développées chez certaines Vanessides et l'apilionides en haut relief en formant des boutons luisants, qui montrent une ressemblance superficielle avee des ocelles.

Les investigateurs qui se sont occupés du stade intrapupal sont beaucoup plus nombreux. Yous avons déjà mentionné ì ret égard les noms de Schäffer et de Van Bemyelex.

Tandis que presque tous les auteurs sont d'accord sur ce point que le dessin intrapupal est plus primitif que celui de l'adulte, nous trouvons chez VAN BemMelen quelques remarques sur lo rapport de ce stade avec le pupal.

Celui-ci ne considère plus le stade intrapupal comme suivant, dans un sens phylétique, celui de la ehrysalide, mais il admet qu'au contraire le premier aurait précédé le dernier. En effet, nous lisons, dans une étude sur les Rhopalocères (4):

"On the contrary, the colour-pattern of the wing-sheath of the chrysalis undoubtedly represents a farther advanced degree of development than the primitive coloration of the wing-rudiments within this sheath".

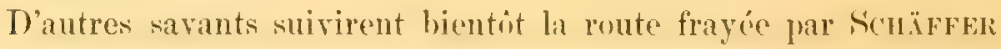
et Van Bemmelen.

Unecri, (74) un de cees auteurs, étudia aussi des espieces de Iranessar, savoir: $r$. urticae et io, pour se former une tout autre opinion que les deux auteurs susdits. Pour lui le dessin dans toute son ¿tendue ("die F'rlderung") se montrait déjà dis le commenerment et c'est l'évolution des couleurs, plus que celle du dessin, qui aurait de la valeur pour la connaissance des relations phylétiques; autre point de vue done, que nous traiterons plus tard (pag. 24).

Quelques détails et argumentations dans cette étude méritent cependant d'être mentionnés. Chez Vanessa urticre il voit au commencement du développement aux petites ailes une couleur rougeitre uniforme ot cherz l'unessu io une couleur blanche. (quelque temps plus tard il voit "sur les parties qui y sont destinées, le 
blanc en partie éliminé par le jaune, ensuite une autre partie du blanc qui n'était pas changée, se colore en rouge, tandis qu'enfin encore d'autres restes du blane se changent en noir. Il déduit de ses résultats une direction identique pour l'évolution des espèces. D'abord, selon lui, l'aile d'une Vanesse a été blanche; ce blanc se changea en quelques endroits en jaune et cette couleur resta intacte dans la suite de l'évolution. Ensuite comme dans l'ontogenèse une partic du blane est remplacée par du rouge, une autre partie par du brun ou du noir; peut-être l'évolution a-t-elle été encore un peu plus compliquée, c'est à dire le noir se serait développé du blanc après avoir parcouru le jaune et le rouge. L'ontogenèse aurait sauté dans ce cas quelques parties de la voie que l'évolution a parcourue.

Il y a beaucoup d'illogismes dans les raisonnements d'URECH.

Selon lui, ce sont les couleurs qui divisent l'aile en parties (Felder) ( es ist die Farbe, welche so zu sagen, zeichnet"): et comme les couleurs se succèdent on serait obligé de conclure que lt dessin parait sucessivement; néanmoins l'auteur constate phus d'une fois que la division en parties ("die Felderung"), le dessin par conséquent, se manifeste d'abord et que les couleurs apparaissent ensuite. Il semble qu'UREcH ait senti qu'on s'attaquerait it ses assertions; du moins il continue ainsi:

,Gegen diesen Satz wird nur ein Misz- und Halbverständnis der I arwin'schen Sätze von natürlicher Zuchtwahl, Anpassung und initierender Natur des Organismus als wichtigster Entwicklumgsfaktor opponieren".

Je trouve aussi un peu trop risqué, de déduire comme Urecı l'existence d'une Tanesse primaire de couleur blanche du fait que les petites ailes dans le stade intrapupal ont au commencement cette couleur. Si cette déduction était juste, les chevaux par cxemple dont le pigment manque d'abord dans la peau de l'embryon, seraient descendus d'un cheval incolore et de cette manière la plupart des animaux auraient en des ancêtres blanes.

Unecr projette une série linéaire phylogénétique des Vanesses et donne les espèces dans l'ordre suivant: 
Tanessa L. album, C. album, xanthomelas, V.album, polychloros, urticae, cardui, io, atalanta et antiopa.

Comme le point de départ est tout autre, nous ne nous étonnerons pas qu'il arrive à des résultats tout à fait différents de ceux de Vax Bemmelen.

Pour Urecir, comme nous avons vu, V'anessa polychloros et urticae étaient des formes plus primitives et Vanessa atalanta et cardui des types plus avancés; pour VAN BEMmelex $V$. urticae et polychloros sont placés à la fin et les deux autres espèces au commencement de la série.

HaAse (27) étudia ensuite les Papilionides savoir: Papilio philenor L., $P$. asterius $\mathrm{L}$., $P$. machaon $\mathrm{L}$., $P$. turnus $\mathrm{L}$. et $P$. podalirius $L$. et établit aussi quant à ce matériel que le dessin se développe successivement.

Tandis que dans son matériel l'aile postérieure se développe plus tìt dans ce rapport que l'aile antérieure, on avait trouvé 'hez Vanessa justement le contraire.

Le fait a quelque signification parce que EnMER avait donné la "loi" du développement postéro-intérieur et le travail de beaucoup d'auteurs, traitant res sujot, des auteurs allemands du moins, était influencé fortement par les opinions de ce savant.

Quelques années plus tard (en 1896 et 1897) des essais assez détaillés ont paru de la main d'Alfred Goldsborovgil Mayer sur le développement des écailles et de leur pigment et sur le dessin des Lafpidoptires diurnes et nocturnes (47, ts et 49). I)ans la dernière étude il communique quelques résultats sur le dessin intrapupal de Callosamia promethea Linn. et Danais plexippus Fabr. Chez ces espèces aussi l'ornementation se développe successivement. P'uis suivent les investigations de la Comtesse Vor Linden $(35,41)$ qui se donna pour but, de rechercher à quel degré des directions déterminées se manifestaient dans le diveloppement du dessin des ailes dans la chrysalide et quelles citaient les relations entre ces directions et le's lois qu'Ermar avait étahlies pour la phylogenèse des Iépidoptères. Pour la défense de ces lois elle a toujours été l'apôtre zêlée. 
Ses travaux, excellents sans doute, sont tout à fait sous l'influence des opinions de l'auteur des lois susdites; aussi se sert-elle de la terminologie particulière de re zoologiste; terminologie qui a été suivic assez longtemps par plusieur's autres élèves avec une grande ténacité, quoique beaucoup d'auteurs aient démontré le grand embarras qu'elle peut causer.

Dans un essai méritoire, couronné par l'Académie des Sciences de l'aris, elle étudie Papilio podativins, P. machaon, Thais polyxena, Fanessa levana et $V$. urticre et elle arrive aussi à la conclusion que le dessin des ailes est composé en ggénéral d'une série d'éléments qui apparaissent l'un après l'autre dans le cours du développement de la chrysalide et qui se fondent peu de temps avant l'éclosion du papillon pour former le dessin imaginal; pour elle, comme pour Ener, les bandes transversales forment le dessin primaire; ces bandes transversales se prolongent d'um bord de l'aile ì l'autre; ces bandes ou lignes peuvent se fondre latéralement, se réduire en taches et s'unir pour former des lignes longitudinales. En rapport avec ce qu'elle a trouvé chez $P$. poclalivius et

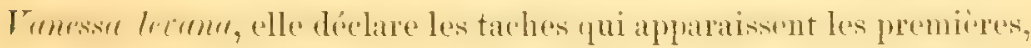
comme une modification d'une bande transversale. Il faut remarquer que nous pouvons déja lire dans le texte allemand: ,dass die Entstehung der primitiven Iä̈ngstreifung ') der Schmetter-

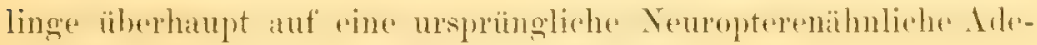
rung der Flügel zuriickgeführt werden muss".

A cause des stades: alebion et glycerion qu'elle observait daus le développement ontogénétique de l'ornementation imaginale de $P$. podulivius elle put constater que ce dernier papillon doit être un type plus avancé que les formes apparentées: $P$. alebion et glycerion. Ces deux espèces se sont arrêtées it un certain point de leur évolution, tandis que $P$. podalivius est allé plus loin (la "génépistase" d'EIMER).

Le même auteur étudia ( 41 ) encore d'autres papillons se rap-

1) Par le terme: „Längsstreifung” l'école d'Ermen à laquelle cet auteur appartient, comme uous l'avons $\mathrm{vu}$, entend ce que tous les autres entomologistes nomment: striation transversale. 
portant.au dessin, de sorte que la liste s'accrut jusqu'à un nombre de 25 espèces, savoir:

A. Rhopalocères:

1. Papilio podalivins L.,

2. Papilio machaon L.,

3. Thais polyxend I.,

4. Thais rumina L.

5. Thecla quercus L.,

6. Tanessa levana L.,

7. Irunessa urticae Is.,

8. Tranessa io I.

9. Tanessa atalanta L.

10. Limenitis sybilla L.,

11. Argymis paphia L.

13. Hétérocieres:

12. Deilephila porcellus L.,

13. Hylophila prasinana L.,

14. Lasiocempa potatorie L.,

15. Gastropache quercus $\mathrm{I}$.,

16. Platisumin cecropia L.,

17. Drepana falcataria L.,

18. Herpyia vinula L.,

19. Notodontre tremula Cl.s.

20. Gonophore deresa L.,

21. Thyativa batis L.

C. Géometrides:

22. Tomosomre linerrive IIb.,

23. Rumia luteolate $\mathrm{I}_{\text {s. }}$

24. Abraxas grossulariata L.,

25. Eupitheciu tamarisciatu $\mathrm{Fr}$.

Dans toutes ces formes étudiées elle trouve comme éléments primaires du dessin: les bandes, les taches ou les zigzags transversaux; chez les Geomitivles des bandelettes plus ou moins étroites qui s'étendaient d'un côté de l'aile à l'autre.

Des bandes plus larges apparaissent dans la chrysalide de 
Gastropacha, Thyative, Hylophila, Sphinx, Satmmia, Thais et P'. packlivius. Chez 'Thais palyxena, I'. machaon, les Vanessu, Limemitis sybilla, Argymis paphir les bandes sont réduites, des leur lébut, à de courts fragments.

Chez Harpyir vinula, Gonophore derasa et Drepana falcataria cette disposition devient de plus en plus apparente à mesure que le développement de l'aile s'avance. Nous trouvons des taches sur les ailes intrapupales d'Argymis paphic, surtout dans l'aile supérieure. Sur l'aile inférieure le dessin commence par des bandes qui, enfin, se décomposent en rangées de taches. Une transformation analogue peut sobserver chez Abraxas grossulariata.

Chez tous les papillons qu'elle avait étudiés, elle dit pouvoil constater que les bandelettes primitives tendent à s'élargir ou ì se fondre et c'est de cette manière que les larges bandes chez les P'apilio, les V'anessa, les Sphinx, les Bombycides et aussi les Géométrides ont tiré leur origine. - Enfin l'aile unicolore peut apparaître.

Le plus grand nombre de bandes fut constaté chez Gonophora, savoir: 16; chez Eupithecia 11; le plus petit nombre chez Grastropacha neustria, Gastropacha potatoria, Hylophila masinana et Thecla quercus Q: 2 ou 1.

Un antre auteur, KURT SMOLin, a fait des recherches en 1912 sur un certain nombre de chrysalides d'Aretice caju, mais dans ces investigations il a plus porté son attention sur la succession des couleurs que sur le déreloppement du dessin, ce qui est regrettable à notre avis. Il mentionne qu'au 21 ì̀me jour après la transformation en chrysalide les ailes avaient déjà leur dessin typique, avec cette exception qu'on voit encore sur le fond blanc des parties pigmentées en rouge. Mais je ne puis reconnaître par son étude, si ces parties ont des contours distincts ou non, si elles se tiennent all cours des nervures, ou si elles se portent indépendamment.

\section{Les recherches morphologiques compares.}

En ce qui concerne les recherches morphologiques e'est surtout EMer (18 et 19) qui tâchait de parvenir à un système de classification 
selon les affinités au moyen du dessin des ailes. Ce dessin était pour lui le critèe le plus sû pour découvrir les legrés d'affinité.

Or il crut avoir observé dans quelques autres groupes du règne animal que le dessin le plus ancien et le plus primitif avait toujours ité celui des bandes longitudinales; la décomposition de ces bandes aurait donné naissance à un dessin de taches et ces taches se seraient transformés souvent par reunion en bandes transversales, tandis que le dernier stade aurait été atteint, quand le monochromatisme entra en scène.

Lorsqu'il rechercha les Papilionides quant à leur dessin, et qu'il trouva aussi chez ces animaux des bandes et des taches, il transporta le schéma susdit de l'évolution phylétique des couleurs de la peau aussi sur les Lépidoptères. Et il est très curieux et sans doute extrêmement embarrassant, qu'il donne aux bandes qui s'étendent du bord antérieur jusqu'au bord postérieur de l'aile, le nom de "Längsbinden", parce qu'elles sont parallèles au corps. Ce parallelisme pourtant se montre hien dans les exemplaires montes, mais n'existe pas du tout en permanence chez les animaux vivants, où les ailes peuvent avoir des positions différentes.

Les lignes ou bandes qui sont perpendiculaires aux bandes susdites, ont reçu par conséquent le nom de "Querbinden".

Cela devient vraiment par trop curieux, parce que de cette maniore les bandes /mugitudimales sont perpendiculaires aux nervures longitudinales et parallèles aux nervures transversales.

Jire voN LINDEN a défendu en ces termes cette nomenclature contradictoire qui, comme ellé dit, ne lui était point du tout sympathique:

„Je ne veux pas changer les noms des nervures, parce qu'ils sont depuis si longtemps cmployés, quiun changement ne pourrait que causer des difficultés sans fin. Mais je ne veux pas non plus copier la nomenclature du dessin sur celle des nervures, parce que je la trouve, au point de rue morphologique, beaucoup mieux choisie que la première. Les temps où on décrivait une petite partie du corps comme un tout n'existent plus; depuis qu'un Cuvier et qu'un Lamarck nous ont montré les relations morpho- 
logiques et physiologiques de tous les organes entre eux, nous ne pouvons plus considérer une partie du corps sans la rapporter an tont".

Enver, par suite de considérations particulières plaça Papilio poralivins, $P$. alebion, $P$. paphius et $P$. glycerion à la tête de toute la série des Papilio comme les formes les plus primitives, puis ceux qui montraient des jonetions transversales entre les bandes longitudinales (c'est à dire d'Enmer) et ensuite les représentants à peu près unicolores, parmi lesquels $P$. colonna avait poussé le plus loin.

Ensuite il accorda une grande valeur à la bande de parade sur l'aile postérieure; bande, très simple dans les types primitifs, se complétant et s'embellissant de plus en plus pour atteindre à la fin son point culminant et.... disparaître.

Comme les formes qu'il considérait primitives avaient onze bandes sur l'aile antérieure, bandes qui, selon lui, avaient une disposition bien définie par leur rapport avec le réseau de nervures, il tâcha de réduire toutes les barres, toutes les taches qu'il trouvait, sur les onze raies: voilà sa théorie des onze bandes ( nelfbindentheorie").

Toutes sortes de déformations de ces onze lignes peuvent s'opérer.

Elles peuvent se fondre latéralement, quelques-unes peuvent disparaitre, elles peuvent se raccourcir ou devenir plus étroites, se décomposer en taches, elles peuvent se joindre l'une à l'autre latéralement ou s'élargir, etc.

Une explication particulière nous est donnée par Ermen du fait que la marge antérieure des ailes montre très souvent un dessin plus primitif que l'autre partic et que les ailes postérieures peuvent déja avoir perdu en partic "les onze bandes", tandis que celles-ci se manifestent encore sur les ailes antérieures dans toute leur étendue.

Il admet une évolution postéro-antérieure; la transformation avance de l'arrière à l'avant, de nouveaux caractères paraissent d'abord à la partie postérieure, ils se meuvent en avant pour être remplacés par d'autres caractères; de cette manière nous avons done pendant l'évolution une ondulation de caractères le long du 
corps et des ailes. C'est la "loi de l'ondulation." Sous certain rapport cette loi a été confirmée par la Comtesse rox Lindes, qui put observer dans son matériel (35) que la formation de la couleur et la fusion des bandes s'opérait dans beaucoup de cas d'arrière en avant et du dehors en dedans, tandis que la marge de l'aile et les nervures se coloraient les dernières.

Les theries d'EnER ont exeré une influence prolongée, surtout par la chaude défense de la Comtesse vor Lindex. Mais l'opposition n'a pas manqqué de se présenter de différents côtés.

C'est par exemple Spuler (70) qui en 1897 démontre l'embarras des termes: Lüngs- et Querbinden, mais s'oppose surtout it la théorie des onze bandes, qui, selon lui, ne plaide pas pour l'exactitude et les soins des observations d'Eryer.

Spuler projette alors un pedigrée des Papilionides que nous ne traiterons pas, et touche aussi it quelques questions d'un caraetìre plus général; il ne s'y prononce pas très distinctement; mais il accentue que l'ornementation primitive des papillons doit avoir consisté en rangées de taches. Il suit de ce qui précède dans son étude, qu'il vise les rangées transversales; il trouve une preuve dans le dessin des Lépidoptires tries primitifs, par exemple Keuzera aesculi.

D'ailleurs, dit-il, on pent entendre à priori par la disposition de l'aile, que le dessin s'est manifesté au début sous la forme de taches ou comme une coloration des nervures.

Une autre critique très acerbe sur le travail d'Ermer était celle de Jordar (31) qui, armé d'une grande connaissance de détails, démontra qu'Ermer avait connu trop peu de formes et qqu'il n'avait pas assez consulté la littérature, pour projeter une série phylétique des papillons - et surtout c'est un des griefs de Jondx qu'Erwer n'avait attuché aucune importance aux autres caractères; qu'il avait accordé, par exemple, peu de valeur phylogrénétigue it la dinposition des nervures et quand Eimer dit, qu'il faisait justement si peu de cas de la disposition differente de la souscostale, parce que cette nervure est tris variable, c'est Jornax qui remarque bien à propos: ${ }^{2}$ comment l'auteur allemand pouvait-il 
donc construire son système sur le dessin, qui est encore plus variable?"?

Au lieu de onze bandes on peut prendre pour point de départ avec le même droit un autre nombre, quand on procède comme Emer, car s'il y en avait trop, une division se serait opérée et s'il y en avait trop peu, une fusion aurait eu lieu ou les bandes auraient disparu. Un autre faible d'ErMER, c'est qu'il a toujours donné des thèses au lieu de faits et qu'il jongle arec les variétés ('t les aberrations individuelles en interpertissant l'une pour l'autro selon son goût:

"In conclusion of this review" c'est ainsi que JokDAN finit sa critique, ${ }^{2}$ which I am sorry to say is mostly destructive, I will not omit to point out that Ermer's rescarehes on Lepidoptera, though full of errors re facts and loose in argumentation, are nevertheless of great interest for the classifier as well as the general biologist. For the very boldness in linguage with which the problems are attacked, the numerous contentions in Artbildung and Orthogenesis, the constant repetition that this or that contention is proved to be correct, will serve to bring the study of Lepidoptera, to which EMser has drawn attention, onwards by instigating others to rerify the facts and examine the arguments. For this Lepidopterologists can only be thankful." La valeur des thèses d'Eimer est done, selon JordAN, qn'on peut les considérer comme point de départ pour des recherches ultérieures sul ce terrain.

En 1890 Drxey (14) avait analysé le dessin des ailes des Nymphalides et comparí ensuite les dessins des divers représentants. Surtout à cause de la méthode qu'il suit, je mentionne ici les éléments différents qui composent les dessins de ce groupe.

Il distingue:

$1^{0}$ une rangée de taches d'un coloris clair près du sommet des ailes antérieures;

$2^{\prime \prime}$ une chaîne submarginale de taches noires arec des centres blens;

$3^{n}$ une bande blanche près du bord costal des ailes antérieures; 
$4^{0}$ Les aréas foncées entre les rangées de taches.

$5^{0}$ Les figures de la cellule discoidale.

Il se construit un Prolovanessa se fondant sur les caractères communs chez les diverses espèces du genre I'ancsia. Ce Protoranessa aurait en, selon lui, une rangée submarginale de taches, chacune avec un centre bleu, et une seconde rangée intérieure à la première.

Selon lui, les lignes d'affinité s'approchent dans la direction d'une Argynnide d'une couleur bleue ou olivâtre foncé, comme la femelle de la forme vivante: Argynnis diana.

Peut-être, dit il, pourrait on reculer encore plus loin et trouver alors un type, dont la couleur foncée aurait été tout ì fait uniforme, dans laquelle aucune différenciation n'aurait eu lieu encore entre la couleur du fond et des taches. Le premier pas en avant aurait ćté ensuite que la couleur de fond se serait éclaircie dans la région des taches entre les nervures, pendant que la couleur foncée se serait maintenue comme des raies ou des taches peu marquées entre les parties plus claires.

Cette différenciation aurait commencé au bord extérieur de l'aile et se serait avancée dans la direction du corps.

Les rubans de la couleur de fond entre ces rangées de taches foncées, seraient devenus d'un brun clair; quelques-uns de ces rubans encore plus clairs; les petites taches communes des espèces de Vanessa auraient pris naissance d'un tel ruban et en auraient formé le dernier reste.

Van Bemmelen non plus n'est pas resté en arrière sur le terrain des recherches morphologiques comparées et c'est surtout les Hépialides, (7, 8 et 9) qui, formant une famille primitive, attirèrent soll attention. Il vaut mieux, je crois, ne pas disserter longuement sur les investigations et les résultats obtenus, puisque cet autenr en donnera lui-même un aperçu, qui paraîtra dans les Publications du Laboratoire de Groningue dans un prochain volume. Je voudrais seulement mentionner quelques points principaux; c'est qu'il a trouvé dans cette famille une ornemeutation qui est limitée parfaitement par le cour's des nervures. 
Les espaces internervuraux, en effet, contiennent des figures en forme de sablier, qui alternent avec des figures elliptiques.

De ce simple motif il put déduixe d'une manière facile et avec une certitude assez grande, les autres éléments des dessins souvent très différenciés.

Si c'est en premier lieu les Papillons diurnes et aussi les IÉpialides, qui ont attiré les investigateurs, d'autres groupes furent aussi le sujet de recherches exactes, comme par exemple l'Arctia caja avee son dessin si variable et quelques formes alliées qui étaient étudiées par KuRT SmoLrar.

De cette étude nous avons déjà mentionné quelques détails. Il est très intéressant d'apprendre ce que l'auteur, qui recherche principalement la variabilité du dessin, a conclu en ce qui concerne l'histoire phylétique de ce Lépidoptère.

Il part des considérations d'Enver, dont il conserve aussi la nomenclature; il croit pouvoir identifier les taches particulières dans l'aile antérieure aux „onze bandes", mais d'une manière qui me semble un peu affectée.

"In einem einzigen Falle", dit-il, nglaubte ich auch am Bande A eine Auflösung konstatieren zu können."

Il considère donc l'Arctia caja comme une forme fortement différenciće, parce que, selon lui, plus les bandes s'unissent, plus l'espèce a atteint un degré supérieur d'évolution. Il se base sur lobservation que, dies lo début, un pigment rouge se produit, pour disparaitre plus tard; il nomme le dessin de l'aile antérieure plus r'écent que celui de l'aile postérieure et confirme ainsi la loi d'évolution postéro-antérieure.

C'est enfin dans notre pays De Meyere (52) qui compara les dessins des Lépidoptères entre eux. Dans la 70ème séance de la Societr Entomologique Nerorlandaise il ne discuta pas seulement cet ordre, mais aussi celui des Diptères.

En ce qui concerne les Lépidoptères, il accorde nombre de directions d'évolution et quant à quelques motifs du dessin, il estime la formation des taches rangées longitudinalement comme primaire, quoiqu'il ne nie pas qu'il faille accorder aussi d'autres motifs 
indépendants pour expliquer les dessins, comme li bordure colorée des nervures longitudinales.

Surtout les rangées de taches le long de la bissectrice des cellules importent beaucoup pour cet auteur.

Quand ces taches sont réduites en nombre, celles qui restent peuvent se placer en rangées transversales, comme on peut l'ohserver chez Kenzera, Abraxas, de nombreuses Aretiides et beaucoup de Rhopalocères e. a. Argynnis.

Les tiches peurent se produire en forme d'anneau et en forme de chevron, comme chez Harpyia vimula et Limantria; les taches peuvent aussi se fondre pour former des lignes ou bandes transversales comme chez les Noctuides.

Parmi les motifs originaires il compte aussi les taches sur les nervures (Hyponomentu, quelques taches d'Abrarus), puis la coloration de la pointe de l'aile, la formation des bandes transversales, la coloration de la marge antérieure etc. Quoique l'auteur, en général, considère ces motifs comme indépendants l'un de l'autre, il ne disconvient pas qu'il n'y ait peut-être un certain rapport entre eux; les taches primaires, par exemple, peuvent être absorbées dans une bande et la même chose peut être admise quelquefois quant à la coloration de la nervure transversale.

\section{Les vecherches expérimentales.}

Weismann a démontré dans un beau mémoire sur le dimorphisme saisonnier, que l'expérience peut aussi jeter un jour nouveau sur l'uffinité des espéces. On savait déjit depuis longtemps que deux formes de Trunessa qui se distinguaient beaueoup en coloration et dessin, ne formaient qu'une espèee, que Vanessa levana et Iromessu morsa étaient le même auimal, le premier représentant la génération d'hiver, le dernier celle d'été.

Pour décourrir le rappurt entre ees deux formes, Grorit DorfMEIster a exposé des chrysalides qui doment naissance ì lit génération d'été à une température de $10-11^{\circ} \mathrm{R}$ et a obtenu én "quelques cas, an lieu de la forme prorse, une forme intermédiaire 
entre levana et prorsa qui était déjì connu comme variété et qui avait été décrite sous le nom de porima.

WeIsMaNY (78), qui ne connaissait pas au commeneement de ses expériences les recherches de Dorfmeister, expose les chrysalides pendant plus de temps à une température beaucoup plus basse $\left(0-1^{\circ} \mathrm{R}\right)$ et obtient de 20 chrysalides 15 formes de porima, dout trois ressemblent beancoup a la forme dhiver, tandis que of individus, nayant pas changé, sortaient en forme-prorsa de leur étui nymphal.

Est-il permis, peut-on se demander, d'attribuer tout a fait la disposition colorée des ailes des papillons à la température qui a régué pendant leur développement, ou y a-t-il encore d'autres facteurs opérants?

Dans le premier cas le changement devrait se produire aussi dans un sens inverse et les chrysalides de la génération d'hiver', quand elles se trouvent exposées à une température plus élevée devraient donner la forme d'été; mais cela n'est à peu près jamais le cas.

Proprement dit, cette espèce u'a pas une, mais deux générations d'été, qui volent en juillet et en août, tandis que la génération levanu vole en avril.

Weismans croyait conclure de ses expériences que la forme levana, la génération insensible donc, était la plus ancienne, et que celle-ci avait constitur pendant la période glaciaire avec ses étés courts et ses longs hivers la scule génération. Mais lorsque plus tard le climat devint plus chand, une seconde génération est survenue et ensuite une troisième. Ces générations parcoururent leur développement dans des circoustances nouvelles et obtinrent en conséquence un autre dessin sur leurs ailes.

La forme levana doit être considérée comme primaire et il dovient compréhensible que la forme plus jeune peut être réduite par un changement des circonstances à la forme ancienne, mais il s'explique aussi que le changement inverse ne se produit pas.

Avec des expériences et des raisonnements analogues Weismaxn arriva à considérer Pieris napi, var. bryoniae - qui, dans les régions 
polaires et les hautes montagnes est la seule forme existante de napi, comme la forme originelle de cette espèce. Pieris napi a dans les zones tempérées plus d'une génération et présente aussi un dimorphisme saisonnier.

Après lui, un grand nombre d'expérimentateurs ont fait des expériences analogues et parmi ceux-ci surtout MERrifieLD, Standfuz, Fischer et Bachanetew jouent un róle important.

Les papillons n'ont pas été seulement exposés par ces auteurs it des températures artificielles, mais on les a mis de beaucoup d'alutres manieres daus des circonstances anormales. On les a fait se développer dans une atmosphere qui possédait une autre proportion d'oxygine ou d'acide carbonique; on les a fait éclairer par une lumiere rouge ou bleue, on a donné aux chenilles une nourriture, à laquelle elles n'ítaient pas habitueés ou on y a mêlé des matieres particulières et enfin on a fait des expériences d'hybriclation.

De toutes ces expériences nous voulons seulement mentionner quelques résultats, en tant qu'ils ont rapport it l'influence de la température.

On peut distinguer:

1e les expériences avec un froid anormal,

2 e $\pitchfork \quad$ avec un froid plus tempéré,

$3 \mathrm{e}, \quad$ avec une chaleur assez grande,

$4^{\mathrm{e}} n \quad$ arec une chaleur excessive.

Arec les expériences sub 2 et 3 on a obtenu souvent des formes qui ressemblaient plus ou moins ì des variétés existantes; le froid transforme les espices ou ruces du Sud en celles qui se rencontrent dans des régions plus septentrionales, tandis que la chaleur est la cause que les formes du Nord acquièrent des caractères semblables a ceux de leurs congénères du Sul. Le froid et Ia chaleur anormales prouluisent souvent des formes aberrantes, ce qu'on peut comprendre par la graude influence qu'une forte altération des circonstances ambiantes peut exercer sur les processus physiologiques et chimiques de l'animal. C'ent surtout arec les Vinesses qu'on a expérimenté. Pour indiquer quelques exemples, Vunesse ataluntu montre, yuand les chrysalides sont soumises a une tem- 
pérature plus b́levée, un claroissement de la bande rouge s'approchant au I'anesser calimhö́ des régions plus méridionales et surtout it la variété vulcanica Got. qui se trouve dans les Iles Canaries.

Dans la forme soumise au froid (savoir: la variété merrifieldi) le rouge est remplacé au contraire par le noir et la bande rouge se décompose en fragments. D'ailleurs les taches costales blanches s'élargissent et des écailles bleues se produisent entre ces taches et la bande rouge.

Il s'opère par la chaleur et le froid anormaux une fusion partielle des taches costales noires et dans ce cas l'aberration lilymene prend naissance.

En ce qui concerne Vanessa cardui, une élération de la température cause une augmentation du rouge et lui donne un luisant plus fort, de sorte qu'elle montre quelque ressemblance avec des formes méridionales, tandis qu'un abrissement cause au contraire un assombrissement et prorluit la forme septentrionale, var. wiskotti. Par les températures, basses ou élévées, mais anormales, on a acquis l'aberration elymi, qui se distingue par la fusion des éléments noirs de l'ornementation du bord antérieur et par la disparition de la figure noire au wilieu du champ basal.

Vanessa io prend par la chaleur un coloris plus foncé quant au brun-rouge, tandis que le bleu disparaît de la pointe de l'aile; par lexposition à des températures basses l'ail disparait, tandis que par la chaleur et le froid plus extrêmes, un noircissement se produit et l'œil des ailes postérieures s'évanouit.

\section{Les aberrations et les anomalies.}

Comme on le sait, les anomalies peurent aroir sourent une grande signification phylogénétique.

Et c'est pour cela qu'il est désirable d'étudier aussi les formes aberrantes des papillons en vue de leur dessin. Un des cas les plus instructifs nous semble être un exemplaire de Gonepteryx rhammi o, capturé par BRYK à Ifyllykylä (11). Les dessous et les dessus de l'aile antérieure portaient une ornementation particuliere; dans quelques espaces internervuraux se rencontraient des 
taches oblongues allongées qui étaient rangées parallelement entre eux et perpendiculairement aux nerrures longitudinales. A cette aberration BRYK a accordé une grande valeur phylogénétique ct non à tort, selon notre avis.

Une autre aberration du même papillon a été décrite sous lo nom de Goneptery.x Hamni ab. Mogressica par GEFr (21). Dans cette forme yuelques taches internervurales bruncs se produisent it la face supérieure des ailes. GEEst la considérait comme une forme plus avancée, plylogénétiquement parlant (,phylogenetisch fortschreitende Form"), quoiqu'il y ait plus de raisons de la considérer comme régressive, de sorte que le nom regressica serait peut-être préférable. En tout cas, on voit qu'il n'est pas recommandable, de mettre son opinion subjective daus un nom nouveau.

Enderlein (17) étudia un exemplaire anormal de T'elea polyphemes CRaver dont la tache translucide manquait sur l'ailo antérieure droite, de sorte que pas une trace n'en était présente, tandis que sur l'aile postérieure seulement quelques fragments des figures environnantes de la tache susdite étaient restés. Le réseau des nervures était également altéré; la nervure transversale, qui ordinairement unit $\mathrm{Cu}_{1}$ arec $\mathrm{M}_{3}$, faisant défaut.

Pour éclaircir ce point, il fit des recherches chez une espèce apparentée et trouva qu'ici un stade se produisait dans le développement du réseau des nervures, dans lequel cette nervure transersale nest pas encore présente. Plus tard elle se constitue, une branche du cubitus s'unissant avec la media. Il considère done la disposition du réseau des nervures de la forme aberrante examinée comme un état parcouru par l'animal ontogénétiquement et phylogínitiquement et de plus il considere toute l'aile susdite comme un organe enrayó dans son développement et resté au stade ancestral; il s'en suit que la tache translucide est un ornement d'une origine très récente.

\section{La couleur et les pignents.}

Il faut faire une grande distinction entre le dessin (ou les motifs qui composent le dessin) et sa couleur; distinction qu'on n'a 
pas toujours observée. Que les couleurs des éléments du dessin aussi bien que celles du fond aient parcouru une évolution, pelsonne, je crois, ne le contestera. Ici, comme pour tant d'autres choses, on a tâché d'établir un schéma pour la phylogenèse, sortint du diveloppement ontoginítique. Et, sans doute, on peut en tirer beaucoup de données pour l'histoire phylétique, mais il ne faut pas perdre de vue, que la plus grande prudence doit être observée. Un stade déterminé de l'embryon ne rappelle pas toujouis une forme originaire des temps passés.

La Comtesse ron Linden $(43,44,41,35)$ a donné plus d'une fois un historique des études concernant les couleurs et les pigments en rapport avec ses propres publications; je puis me restreindre en me référant aux points principaux.

Par les recherches de Hopkixs, Perry, Coste, Poultor, Urech (75) il a déji cité montré depuis longtemps quion peut distinguer deux rubriques de couleur's:

$1^{0}$ les couleurs naturelles ou pigmentaires, dues i un pigment,

$2^{0}$ les couleurs optiques, dues aux phénomènes d'interférence de la lumière.

En outre, il y a des couleurs qui doivent être considérées comme une combinaison des deux groupes.

Hopkrss a trouvé dans les ailes de Gonepteryx rhammi un pigment jaune qui était soluble dans l'eau et qui était un dérivé de lacide urique. Pour un pigment vert qui se trouve aussi chez quelques papillons, Griffitrs a établi la formule empiriqne $\begin{array}{lllll}\mathrm{C}_{11} & \mathrm{H}_{12} & \mathrm{~A} z_{8} & \mathrm{O}_{10} & \text { (acide lépidoptérique). }\end{array}$

URECH aussi a fait des recherches sur la nature chimique des pigments (75), et arrive, comme Hopkins, à la conclusion qu'ils ont des affinités arec l'acide urique. Sur l'origine de la couleur les auteurs ne sont pas tous d'accord. Quelques-uns considèrent les pigments comme dos matières animales, c'est à dire, comme des produits de l'assimilation et de la désassimilation du corps du papillon. D'autres admettent que ces pirments sont originares des plantes qui ont servi de nourriture aux chenilles. Les matieres, dans ce cas, cherchent une voie par les parois de l'intestin 
et sont conduites, par le sang, aux écailles. En rapport avec cette dernière hypothise, des expériences trìs remarquables ont été faites par Portus avec les chenilles d'Agrotis prombr qu'il éleva a l'abri de la lumiere; quand il les nourrissait de feuilles vertes ou ítiolées, elles pouvitient développer leurs couleurs vertes et brunes d'une manire normale, tandis que, quand les chenilles étaient nourries de nervures blanches de chou, ces couleurs n'apparaissalient pas.

Il en conclut que la chenille a le pouvoir de changer la chlorophylle, la xanthophylle et l'étioline en pigment. On at démontré en effet que le sang contient de la chlorophylle et d'après Mayer (46) ce sont les corpuscules du sang qui causent la coloration des écailles.

C'est surtout Mlle von LINDEN qui a essayé d'éclairer cette question. Elle trouve chez les Vanesses que le pigment vert du contenu de l'intestin se change en rouge peu de temps après la transformation ev chrysalide. Ensuite clle a démontré le pigment rouge dans les cellules des parois de l'intestin et quelque temps plus tard elle trouve cette matière colorante daus les espaces cutre les lames membraneuses de l'aile (dans les canaux sanguins donc), qui se trouvent dans les nervures et qui entourent les trachées.

Puis elle l'a recherchée dans les cellules qui forment la lame membraneuse de l'aile et par conséquent aussi dans celles qui donnent uaissance aux écailles, tandis que, quelque temps apris, les pigments s'amassent dans les écailles, lorsque les cellules qui les ont formées en sont privées totalement.

Elle admet done que les pigments des écailles des Vanesses sont des dérivés de la chlorophylle, qui est porté dans lintestin arec la nourriture.

Comme le pigment rouge se produit déjà dans l'intestin, il s'en suit pour l'auteur que le rouge a été la couleur primaire des Vanesses. Mais quoique ce rouge se manifeste au début du développement, toute l'aile n'est pas colorée de cette manière. Ello l'explique en admettant que les écailles des endroits qui prennent un coloris foncé, se sont développées plus tard.

Lussi peut-elle se reneontrer sous quelques rapports avec PIEPELS, 
(5) qui prétend que li couleur primitive des papillons a été rouge. Il faut ajouter que dans une autre étude (39) elle s'éloigne de Prepers en considérant le rouge comme un mélange de jaune et de rouge; de ces couleurs le jaune serait plus ancien que le rouge.

Mais elle ne peut admettre que le rouge et le jaune soient les couleurs primaires de tous les lepicloptires, puisquiun pigment rouge ne se produit pas chez un microlépidoptère, examiné par elle, savoir: Botys urticata; ici la couleur primaire devrait être vert ou vert-brun. Elle trouve dans le développement ontogénétique lordre suivant des couleurs: jaune, orange, carnin, cinabre, brun-rouge et noir; c'est le même ordre indiqué par Ermer pour l'histoire phylétique.

Sroular trouve comme succession des couleurs chez Arctia caja: blane ou jaune-brun, rouge passant au brun ou au noir; de cette lerniere couleur se serait développé le bleu comme couleur optique.

Ropport entre le dessin des Lépidoptères et celui des autres ordres d'Insectes.

Tant la littérature est riche, quant au dessin des papillons, tant elle se montre paurre en vue du rapport des ornementations des différents ordres entre eux.

Parmis le petit nombre d'auteurs qui se sont occupés de ce sujet on trouve de noureau la Comtesse vor Linder.

Dans son étude française: "Le dessin des ailes des Lépidoptères" il y a un chapitre intitulé: "Le dessin des Névroptères, des Orthoptires, des Hémiptires, les Homoptires et des Diptires comparé au dessin des Lépidoptères."

Traitons d'abord les Trichoptères.

Dans ce groupe elle distingue:

$1^{0}$ Ceux dont la lawe membraneuse de l'aile est incolore, mais les poils sont colorés, comme par exemple dans les espèces suivantes: Agapetus Curt., Psychomia Latr., Setodes Ramb., Goüra Hoff., Aspatherium Kolen, Irichostoma Pict., Sericostoma Latr.,

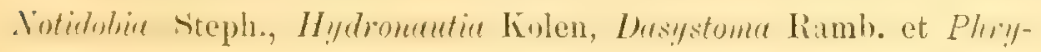
ganea $\mathrm{I}_{\mathrm{s}}$. 
$2^{n}$ Ceux ehez qui la membrane ailaire porte l'ornementation: Yeuronia, Hulesus flacipennis, Limnophilus striole et L. viber Curt.

On rencontre dans cet ordre aussi des bandelettes étroites qui se prolongent dins une direction transversale et qui se décomposent souvent en taches.

C'est le cas chez Neuronia reticulata L., où les bandelettes se décomposent dans la région du bord antérieur et postérieur. Les taches tendent à se fondre en un dessin réticulaire. Sur l'apex de l'aile postíricure nous rencontrons des restes de bandelettes. Les bandes longitudinales ru'elle trouve chez Hulrsus fluripennis sont composies de petits fléments transersaux: les fragments de bandelettes transversales que l'on peut encore reconnaître sur l'apex de l'aile.

Chez Halesus menctatus elle trouve six bandes plus larges, formant des dents aiguës sur chaque nervure.

Quant it lil eonclusion de ces recherches, elle la résume comme suit:

„Le dessin primitif est disposé en fines bandelettes transversales 1). Tous les autres types dérivent de ce dessin primitif qui se transforme cu rangées de taches, en bandes transversales, en dessin réticulé ou bien en de larges bandes longitulinales qui, en se fondant, conduisent à des ailes uniformes."

Et elle continue: „Comparé arec le dessin des Papillons, celui des Trichopteres se compose d'un plus grand nombre d'éléments plus fins et il me semble que de cela aussi vient que le dessiu des Trichoptires est plus variable que celui des Papillons. Nous observons même une asymétrie du dessin sur les ailes du même individu. Chez les Trichoptires manquent aussi généralement des bandes bien localisées, quoique plusieur's plices léterminées des ailes, le ptérostigma, par exemple, aient de préférence une coluration très intense. Ces différences entre le dessin des Papillons et celui des Trichoptères nous autorisent à dire qu'arec la simplification du dessin et lil réduction du nombre des éléments qui le composent, celui-ci devient plus symétrique, mieux déterminé

1) Elle emploie le termo: longitudinales (Enrer). 
et localisé." Puis clle traite le dessin des Nivroptires planipennia Elle y compte e. a. anssi les Panorpes et les fourmilions (1/yro meleon), Ascalaphus ete. Ces formes ont aussi le plus souvent des bandes transversales; les deux premiers ordinairement quatre; 10 nombre qu'on rencontre aussi chez Nemoptera $\cos ^{\circ} \alpha$.

En ce qui concerne les autres groupes: les Orthoptères, les Psendonévroptires, les Hémipteres et les Diptires, on peut trouver dans tous ces ordres des formes à bandes transversales.

Des groupes examinés, les Ephémérides, selon l'opinion de l'auteur, sont les plus simples quant à la forme des ailes et à la disposition des nervures. C'est aussi dans ce groupe que nous rencontrons les dessins les plus primitifs et les plus simples. Les matières colorantes n'apparaisscnt que sur les nervures transversales et s'étendent en lignes parallèles du bord antérieur jusqu'au bord postérieur de l'aile.

Quelques bandes sont entrecoupées en zigzag par des réductions dans le systeme de nervures transversales.

Done, chez tous les insectes, selon l'auteur, les bandelettes itroites transversales constituent le dessin le plus primitif. D'un côté ces bandelettes peuvent se fondre pour former des bandes plus larges ou de grandes taches et au dernier stade un dessiu unicolore. Dans une autre direction le développement conduit à un dessin réticulé, tandisque quand la production de pigment devient moins grande, les bandes se décomposent en rangées de taches ou sont réduites dans leur longueur. Quand cette tendance se déreloppe encole plus loin, les ailes deviennent incolores.

Une deuxième étude que nous avons déjàt citée et qui vise une compillaison entre les ordres différents, est celle de DE Mryera (5)2, 5:3).

Mais comme cette étude a été lue dans une assemblée, je dois me contenter d'un simple compte-rendu'). D'abord l'orateur parcourt l'ordre des Diptères; parce que la plus grande partie a des ailes incolores, il considère cet état comme primitif et il estime qu'un dessin déterminé dans cet ordre est un phénomène secontaire. Fnsule il indique les lignes différentes dovolution

1) Quaut à l'étude elle-même, je l'ai reçue plus tard (voir le dernięr chapitre). 
qui ont conduit à un dessin et comme, traitant des Lépidoptires, il accorde à peu près à cet ordre les mêmes directions d'évolution que pour les Diptères, je vais donner ici son schéma:

I Figures en rapport avec le contour de l'aile:

la coloration de la marge antérieure, de la pointe, de la racine de l'aile, de la marge postérieure.

II Figures en rapport avec le réseau des nervures:

la bordure des nervures transversales et des nervures longitudinales; la coloration du point de bifurcation, des extrémités des nervures longitudinales; la striation ou les taches le long de ces nervures; la formation d'un stigma, la formation des taches aux centres des cellules; des rangées de taches sur la bissectrice des espaces internervuraux (quand ces taches se divisent, il y a une double rangée).

III Figures plus ou moins indépendantes:

la formation des bandes transversales; l'assombrissement diffus; la coloration nuagense; la formation d'anneaux (par la production de centres clairs dans les taches ou les bandes) et de petites taches translucides;

IV Coloration en teinte laiteuse ou blanchatre, en grande partie tombant sous les cas susdits;

V Couleurs causées par des appendices particuliers:

des écailles chez les Culicides, des poils chez les Asilides, de petites barres ou grains chez Psilopus anthracinus et quelques Tripétines.

Il trouve très remarquable que quelyuefois l'évolution dans une même famille, où se rencontrent des dessins, ait pris des directions diverses, mis surtout qu"il semble assez indifférent quelle direction fut prise, quand une formation de pigment se produisait.

Il rencontre, par exemple, dans les Tipulides tous les motifs possibles et de méme dans les autres groupes comme: Anthraeines, Tabanides, Trypétines, Ortalines, Lauxaniines etc.

I.es Névroptères sont aussi, d'après cet auteur, divergents à légard de leur dessin au lieu d'etre simples et uniformes. Beauroup de directions présentes dams les Dipteres se produisent aussi 
dans les Névroptères; dans les Panorpides il observe des bandes transversales comme le motif le plus ordinaire.

Quant à l'opinion que cet entomologiste se formait du dessin des Lépidoptères, nous l'avons déjà indiquée.

\section{B. Les opinions d"aujourd"hui sur le dessin primitif.}

Nous avons vu dans l'Introluction deux theories contradictoires sur le dessin primitif.

Un des groupes d’investigateurs cousidire comme tel, un systime de bandes transversales, qui s'étendent du bora antérieur jusifu'au bord postérieur.

A ce groupe appartiennent ou appartenaient e. a. EIMER, La Comtesse ron LINDEN et Srolian.

Avec un aplomb extraordinaire Erher avait donné ses „onze bandes" comme la clef qui résoudrait tout l'énigme du dessin.

"Diese von mir vorzuführenden Thatsachen sind derartige, dass auch Gelehrte, welche bisher ganz anderen Auftassungen huldigten und sich darin festgelegt haben, sich ihnen nicht werden entziehen kïnnen, obschon sie sich häufig am hartnäckigsten gegen das True wehren", dit-il dans son ouvrage: "Die Artbildung und Verwandtschaft bei den Schmetterlingen" (18).

Ille vox LINDEx, quoiqu'elle ait défendu les opinions d'Ermer d'une manière magistrale, a dû abandonner enfin le nombre de nol1ze".

Mais dans les derniers temps SMolias, comme nous avons $\mathrm{vu,}$ s'est attelé à la même tache et, cô̂te que coûte, il parvint à tirer de son matériel le nombre d'Ener.

Quelle est la cause du phénomène que le pigment se dépose de préférence en bandes transversales?

Nous lisons dans l'étude (41) de la Comtesse vor Lindex, qu'elle admet que cela s'explique par le cours de petits canaus très fins, des canaux sanguins, dont elle a pu démontrer l'existence au début de l'état chrysalidaire dans les ailes de quelques papillons.

Comme le sang conduit le pigment, les canaux sanguins sont 
naturellement les endroits destinés d'avance au dépot du pigment. Dans un seul cas, chez Papilio podalirius, elle vit; au début du développement des ailes chrysalidaires, outre les canaux sanguins dans les nerrures longitudinales, d'autres canaux qui étaient perpendiculaires ì res nervures. Elle vit des canaux analogues, mais très étroits, sur la bissectrice de quelques espaces internervuraux chez lanessu io et morsa. C'ette disposition expliquerait les raies colorées longitudinales au milieu d'un espace internerrural.

Pour l'auteur la présence des bandelettes est done liée an cours des canaux sanguins.

Chez les Bombycides elle croit avoir trouvé une autre cause de la disposition du dessin, c'est ici le relief de l'aile et ce relief est la conséquence du relief de l'abdomen. Elle trouve sur la chrysalide de Bombly quercus des parties renfoncées, correspondant aux parties enfoncées de l'abdomen, aux endroits entre. les anneaux. Une bande claire correspond aux parties evfoncées de l'aile, les champs obscurs correspondent aux parties élevées.

Ce relief aurait changé la distribution du sing et aurait done influencé sur la pigmentation.

Et enfin, elle admet ce que d'autres auteurs aussi avaient díji prétendu, que quelques figures se seraient produites par le contact avec les organes colorés qui se trouvent au dessous de l'aile et qui auraient donné leur pigment aux organes superposés.

Il ne serait pas juste de ne pas mentionner la tentative de Gebinatio (22) d'expliquer la disposition du dessin par voie physicochimique.

Il ne voit dans les bandes transversales et longitudinales et dans celles qui environnent les taches en forme d'œil, que des anneaux de Liesegara. Ce savant a pris une masse gélatineuse, imprégnée d'une certaine matière chimique et il a fait diffuser dans cette masse à partir d'un point léterminé une autre matière chimique, qui forme un précipité avec la première.

Ce précipité se dépose cu anneaux concentriques autour du point de départ et on voit une alternation régulière des parties où ce précipité s'est amassé avec celles où il ne se forme pas. 


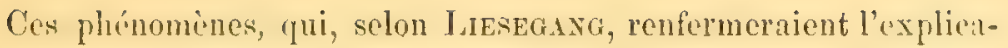
tion de la coloration du minéral agate, donnent selon GEBirardT l'explication de la coloration des papillons.

"Zur Zeit der Ent-tehung der Flügelzeichnung, also wïhrend der Puppenruhe, stellt der Schmetterlingsflügel in letzter Linic eine dünne Lage von Colloiden zwischen zwei wohl im wesentlichen als indifferent anzusehenden Chitimplatten dar. Die Zellgrenzen spielen für unsere Betrachtung keine Rolle,"

Cette manière de présenter un organe d'animal est à mon opinion un peu profane; de cette façon tout le corps ne serait nin letzter Linie" "qu'un pâton de gelée daus une peliseule indifférente. S'il y arait une part de vrai dans cette façon de présenter les choses, les anneaux de Liesegang devraient s'étendre autour des canaux et des lacunes qui se rencontrent dans l'aile mais non arec la racine de l'aile comme centre.

Contraire à la théorie des bandes transversales, est celle qui considere les rangées longitudinales internervurales de taches comme les ornementations primitives. Parmi ceux yui défendent cette opinion on peut compter Spuler, Mayer, Scuröper, De Meyere et il y a quelque temps aussi van Bemmelen. Zeuzera pyrina est sous ce rapport une forme des plus primitives.

Quand je m'occupais d'après les conseils de M. le Prof. van Bemuelen, d'examiner les dessins des Cossides, j'ai aequis la conviction que les traits transversaux, petites lignes qui s'étendent d'une nervure longitudinale à la voisine et qui sont placés pour la plupart perpendiculairement à ces nervures, devraient être considérés comme les éléments les plus primitifs.

Je présenterai dans un prochain chapitre les preures que je pense pouvoir donner. J'ai déjà résumé mon opinion dans une étude qui a paru, il y a peu de temps, dans les Comptes-Rendus de l'Académie Royale des Sciences à Amsterdam (10).

En apparence les deux théories, celle d'Eimer c. s. et celle des antres, sont diamétralement opposées. Si nous partons cependant des traits internervuraux, les bandes d'Eimer et les rangées de taches de la partie adverse ne paraissent être que de petites 
modifirations du motif susdit, mais dans des directions differentes, de sorte que les deux parties n'ont qu'un seul pas à faire pour être parfaitement d'accord.

\section{CHAPITRE II.}

\section{DESCRIPTIONS DE DESSINS.}

\section{A. Matériel et méthode.}

Le matériel sur lequel j'ai fait mes observations, en ce qui concerne les Cossides aussi bien que les autres familles, se troure en partic dans les belles collections de Lépidoptives paléaretiques de KAllexbaci et de De Bork et Kooy au Laboratoire Zoologique à Groningue.

Pour une autre partie, je pourais disposer du matériel de Cossides d'une collection que le Iaboratoire avait achete autrefois de Stcunfinger et d'une petite collection du Jardin Zoologinue d'Amsterdam.

Grâce à la grande bienveillance de M. le Prof. DE Merere à Ansterdam, jai recu quelques Trichopteres de sa propre collection, tandis que le Musée d'ITistoire naturelle it Leyde m'a confié aussi quelques représentants de cet ordre. A tous ceux qui ont facilité mes études en me prêtant des spécimens j'exprime ici ma grande gratitude et surtout en premier lieu à M. le Prof. VAx BeMnelex, 'fui m'a toujours aidé par sa raste connaissance de la littérature et aussi sous beaucoup d'autres rapports.

Je ne puis pas non plus négliger d'adresser un mot de remerciement i M. vaN EGMond, préparateur au Laboratoire, pour les belles photographies qu'il a faites.

Ma méthorle de travail est assez simple; elle comprend l'analyse exacte du dessin pour parvenir à des conclusions en comparant les diverses analyses. Je crois que la méthode des descriptions minutieuses est la seule qui doive être suivie dans l'étude de la phylogenise du dessin. Cette méthode est déja utile en tant que l'attention est attirée sur des détails qui plus tard paraissent avoir de la valeur et qui sans cela seraient négligés. 
Les photographies des papillons ont été retouchées, mais toujours avec l'objet sous mes yeux, de sorte que je suis sûr que chaque raie ou tache se retrouve en effet sur l'individu.

\section{B. La terminologie de l'aile.}

En décrisant les dessins, je dois indiquer fréquemment des parties ou des nervures de l'aile. Et comme la nomenclature n'est pas tunjours la même dans la littérature lépidoptérologique, nous donneruns, pour éviter toute épuivoque, une courte description topographique de l'aile.

Aux deux ailes on peut distinguer:

$1^{0}$ le bord antérieur ou costal,

$2^{\circ}$ le bord externe,

$3^{0}$ le bord postérieur ou anal.

Ces inots sont aussi employés par HANDLiRscir, par exemple, dans le Traité d'Entomologie (bs). On trouvera une autre terminologie dans Oudemaxs: De Nederlandsche Insecten" (55). C'eluici parle dans le même ordre du bord antérieur, postérieur et intérieur. Dans l'étude de cet auteur, indiquée sous 56, il donne les termes: bord costal, extérieur et anal.

Le lieu d'insertion de l'aile au corps s'appelle la racine; là où le bord antérieur et extérieur s'unissent, se trouve le sommet de l'aile, et où le bord extérieur et postérieur se rencontrent: la queue de l'aile.

La membrane ailaire est soutenue par des nervures; entre ces nervures nous trouvons les plis bissectrices.

Les nervures sont pour la plus grande partie longitudinales, quelques-unes seulement sont transversales.

Nous placerons, comme C'Osstock, le bord antérieur au même rang que les autres nervures, de plus paree que LxDERLEIN (17) a démontré qu'il peut contenir originairement une trachéc bien développée.

Nous arons done pour l'aile antérieure, en partant du bord antérieur:

$1^{0}$ la costa, la nervure costale. ')

1) J'ai emprunté en partie ces coing de Henneavy (28). 
$2^{0}$ la subcosta (= I de SPULER), la nervure sous-costale; celle-ci ne se bifurque que rarement et aboutit dans le bord antévieur.

$3^{0}$ le radius (= II de Spuler), la nervure humérale, brachiale ou radiale; celle-ci a ordinairement cinq branches qui aboutissent en partie dans le bord antérieur, en partie dans le bord externe; la racine commune des quatre dernières avec ces branches elles-mêmes est nommée le secteur de la radiale; la première branche conserve le nom de radius.

$4^{0}$ la media, mediana ou médialis (= III de Spuler), la nervure médiane; de rette nervure la partie basilaire disparait, excepté dans les papillons les plus primitifs (Hépialides, Mieroptérygides, Castniides), où cette partie se maintient.

La nervure médiane se divise ordinairement en trois branches.

$5^{\circ}$ le cubitus (=IV de Spderer), la nervure cubitale antérieure, qui se divise seulement une fois et aboutit done en deux branches.

$6^{0}$ Les nervures anales $(=\mathrm{V}$; a et $\beta$ de Spuler); la scconde s'appelle aussi la nervure cubitale postérieure; la troisième porte dans ce cas le nom d'anale.

Celles-ci se dirigent vers lo bord postérieur.

Les parties basilaires du radius et du cubitus limitent un champ, fermé plus ou moins par les nervures transversales. Ce champ c'est la cellule médiane ou discoidale ou area médiane.

Les surfaces ailaires yui sont eomprises entre les nerpures, ont reçu les noms de cellules ou areae.

La surface entière peut être divisée en deux parties qui, en vue du dessin, se comportent trìs souvent d'une maniere différente.

Cette division a été proposée par Spuler, qui dénomma les deux parties par les termes: Spreiten- und Faltenteil, ce qu'on pourrait essayer de traduire en français par partie limbaire et partie en éventail.

La ligne de démarcation de ces deux parties est la nervure $V$ de Sptuer (la cubitale postérieure); Spt lekr comptait celle-ci encore ì la partie limbaire. A la partic en éventail appartiennent done les deux dernières nervures anales. Quoique Exdmenas ait démontrí que cette division, ontogénétiquement parlant, ì canse de 
la disposition des nervures, n'ait point droit d'existence et qu'il n'y ait pas de limite entre la média et le cubitus, j'employerai néanmoins par la suite les termes susdits, parce que la partie en érentail, en ce qui concerne le dessin, se comporte tout autrement que le reste de l'aile.

La nervure qui suit le cubitus, est souvent oblitérée et remplacée par un pli. Présente, nous la nommerons nervure anale I (=V de SPULER), absente nous parlerons du pli anal.

Dans les Cossides on peut rencontrer aussi bien la nervure que le pli; le dernier, par exemple, chez Langsdorfia et Acousmaticus (61).

Le réseau de nervures de l'aile postérieure correspond à celui de l'aile antérieure; seulement le radius a deux branches au lieu de cinq. (Exceptious: les Hépialides et Microptérygides, ou il y en a cinq.)

Dans l'aile antérieure nous avons done:

$\mathrm{C}$, SC, $\mathrm{R}_{1}, \mathrm{R}_{2}, \mathrm{R}_{3}, \mathrm{R}_{4}, \mathrm{R}_{3}, \mathrm{I}_{1}, \mathrm{M}_{2}, \mathrm{I}_{3}, \mathrm{Cu}_{1}, \mathrm{Cu}_{2}, \mathrm{An}_{1}, \mathrm{An}_{2}, \mathrm{An}_{3}$.

Dans l'aile postérieure ordinairement:

$\mathrm{C}, \mathrm{SC}, \mathrm{R}_{1}, \mathrm{R}_{2}, \mathrm{II}_{1}, \mathrm{MI}_{2}, \mathrm{M}_{3}, \mathrm{Cu}_{1}, \mathrm{Cu}_{2}, \mathrm{An}_{1}, \mathrm{An}_{2}$ et $\mathrm{An}_{3}$.

\section{La famille des Cossides.}

Les Cossides, appelées aussi Zeuzérides, comprennent, selon KinbY (3ii) et SrIT\% (69) environ quarante geures et plus de 200 espèces; à peu près chaque année ce chiffre s'accroit par un certain nombre de nouveaux genres et de nouvelles espèces (voir Scilles (64), Rothochud (62)). Les chenilles vivent dans le bois; re lit le nom allemand: "Holzbohrer" et le nom vulgaire: Cossus ronge-bois pour une des espèces (Cossus cossus).

Aussi Lxonet (46), qui en 1760 donna un traité anatomique de la chenille de Cossus cossus, la nomme-t-il , la chenille qui ronge le bois de saule."

D’après Seitz et KinBy la famille a ses représentants répandus par toute la terve - 50 à 60 espèces dans la Région paléarctique et américaine; un peu plus dans la Région indo-australienne et un peu moins dans la Région africaine, quoique dans 
cette dernière partie de monde, il se trouve sous ce rapport encore beaucoup de "terra incognita".

Quelques espèces sont rares, d'autres, comme Zeuzera se trouvent dans une grande partie du globe et sont très communes.

KIRBY donne la diagnose suivante:

Larva: almost naked, with a few scattered hairs; boring galleries in growing wood, or living in reeds.

Pupa: Formed in galleries, and furnished with spines by which it can push itself forward; sometimes enclosed in a cocoon of wood or silk.

Imago: of moderate or large size, with the mouth-parts rudimentary, the antennae pectinated to the middle or to the tip, at least in the males (d'après Scunưs il y a aussi des mâles aver des antennes nou-pectinées, par exemple Mircora), frenulum present, consisting of several bristles in the female; cells of all the wings bisected by a nervure, which forms a shorter or longer fork towards the extremity; fore-wings with an accessory cell above the upper extremity of the discoidal cell; hindwings with three submediau nervures. Body very stout, or long and tapering; female provided with an oripositor.

\section{Cossus cossus I.}

$$
\text { (Pl. I, fig. 1-7) }
$$

\section{Le dessus de l'aile antérieure.}

Nous trouvons le long du bord antéricur un plus ou moins grand nombre de traits transversatux foncés; ceux-ci se prolongent queliuefois à travers quelques cellules et se prísentent comme de petites barres parallèles, qui cependant ne sont pas tout i fait droites, mais dont les fragments, dont elles sont composées, peuvent être plus ou moins courbés. Quelques-uns de ces traits sont assez foncés, d'autres, au contraire, plus clairs, tandis que les espaces qui restent entre ces barres sont colorés d'un brun grisâtre ou d'un gris blanchâtre.

Quelquefois les barres claires et foncées alternent régulièrement, 
quelquefois on ne peut remarquer aucune régularité; dans le premier cas nous trouvons à plusieurs reprises deux traits foncés, séparés par un champ plus clair avec une bande un peu plus foncée au milieu; dans le dernier cas nous observons souvent un grand nombre de traits parallèles de la même teinte, très rapprochés l'un de l'autre.

La plus grande partie de la surface ailaire est ornée de traits foncés analogues qui se maintiennent toujours dans le cours des nervures; ils s'itendent d'une nervure longitudinale à la voisine, sont placés perpendiculairement à ces nervures ou forment un autre augle avec celles-ci. (Juelques-uns sont un peu courbés, d'antres sinueux ou ramifiés, ou bien une barre de jonction se produit ça et lì entre deux de ces traits.

Les traits ne sont pas partout de la même largeur; quel. ques-uns sont plus larges au bout, d'autres au milieu; ils ne sont pas non plus du même coloris. Quelques-uns sont presque noirs et marqués distinctement, taulis que d'autres sont plus effacés et sautent peu aux yeux. Dans le champ basilaire ils ne sont pas si développés, quoiqu'ils n'y manquent pas tout à fait.

Le bord postérieur est orné d'un motif analogue; on y trouve aussi une alternation d'éléments plus clairs et plus foncés. Ce qu'on voit du premier coup d'oil, e'est que certains traits transversaux ont une tendance à se joindre pour former des lignes trimsversales, qui parconrent une plus ou moins grande partie de l'aile. Chez Cossus ces lignes ne sont pas très constantes; ordinairement il $\mathrm{y}$ en a trois. Dans un des exemplaires les lignes étaient si peu développées quielles rehappaient presque à l'attention (Pl. I, fig. 5).

Dans un autre exemplaire la ligne la plus longue, celle du milieu, s'était rompue et les deux bouts avaient glissé, pour ainsi dire, l'un contre l'autre. Dans un troisième se trouve à la place de cette ligne une bande bornée des deux côtés par une ligne tandis que lespace intrijacent cst coloré d'un ton assez foncé. La plus longue de ces lignes s'étend sur une partie assez importante de l'aile, quoiqu'elle ne soit pas complete; un certain 
nombre d'autres lignes au contraire ne parcourent que deux ou trois espaces internervuraux.

Ces lignes paraissent titre composées de fragments qui se restreignent à un seul espace. Ces fragments sont plus larges que les autres traits internervuraux du dessin. Quelquefois ils sont accompagnés d'une bordure blanche, de sorte qu'ils devieunent plus distincts.

La direction peut varier aussi; tandis que dans un excmplaire assez clair de la collection de KaLLexbach la grande ligne médiane et la petite qui commence environ au sommet de l'aile autérieure font un angle assez grand, ces deux lignes étaient à peu près parallibes dans un autre, un triss bel exemplaire de la collection de De Boer et Kooy.

Dans une partie de l'aile, le long du bord externe, nous trouvons un autre motif: le motif r'éticulaire. En s'éloignant des nervures, les traits se courbent et s'unissent daus l'espace internervural pour former une maille. Des coins de eette maille d'autres traits colorés prennent naissance, pour enfermer d'autres espaces polygonaux. Les dimensions des mailles ne sont pas du tout toujours les mémes; tantôt elles ocrupent la moitié de la hauteur d'une cellule; tantôt un tiers ou un quart. Souvent on ne peut pas constater de régularité.

De même que les traits transversaux, le réseau coloré peut avoir également des teintes diverses entre le brun-clair et le uoir. L'espace enfermé est souvent blanchâtre, tandis que le centre peut avoir un coloris plus foncé.

Quand on compare les ailes de différents exemplaires entre cux, on ne remarque pas seulement que les réseaux colorés de deux cellules homologues peuvent être tris différents, mais on voit aussi qu'un des exemplaires peut posséder dans une cellule détermince le motif réticulí, tandis que dans la même cellule de l'autre le motif des traits internervuraux transversaux se rencontre.

Nous avons vu que ces deux motifs se partagent la surface de l'aile; l'étendue de chacun n'est pas tuujours la même, quelquefois le réseau coloré occupe toute la moitié distale de l'aile, dans un 
autre exemplaire il se borne à une marge le long du borl externe. En ce qui concerne la couleur du fond, celle-ci est principalement d'un gris foncé. On peut observer une tendance à la formation de champs colorés de diverses nuances.

Le champ basilaire est blanchâtre aussi bien que la pointe et une marge le long du bord externe, tandis que la partie moyenne est colorée d'une teinte plus foncée. Dans les différences de nuances entre ces trois parties il y a toutes sortes de variations.

Le champ moyen foncé s'élargit dans la direction du bord postérieur et est délimité en dehors par la grande ligne noire, dont nous avons parlé; la délimitation en dedans est moius marquée et beaucoup plus incomplète.

Dans la partic postérieure de ce champ on remarque quelques taches blanchâtres.

Aux bouts des nervures, pour une grande partie sur la frange du bord externe, nous rencontrons des taches assez vagues, mais tri's rógulieres avee un centre blanchàtre. ('es taches sont ívidemment en rapport avec le réseau coloré.

\section{Le dessus de l'aile postérieure.}

Le dessus de l'aile postérieure est moins différencié que celui de l'aile de devant, et a une teinte plus égale. Sur le fond brungris, qui est seulement un peu plus clair sur la marge externe, on trouve un dessin plus ou moins distinct, qui en tout cas saute moins aux yeux que celui de l'aile antérieure. Eu gínéral cependant, les dessins des deux ailes ont beaucoup de ressemblance et varient d'une façon analogue. Quand nous trouvons sur l'une des ailes une grande extension du motif réticulaire, nous rencontrons la même chose sur l'autre aile. Dans la direction de la rilcine tont ornement disparait. Cette partie est couverte d'écailles élongées en forme de poils et partout où ce cas se produit, un dessin unicolore se montre généralement. La partie en íventail de l'aile présente peu de traces de dessin, on les rencontre seulement dans le voisinage de la partie limbaire. Ce qui est remarquable, e'est que la marge antérieure de l'aile postérieure est 
ornée de la même manière que celui de l'aile antérieure, quoique le dessin soit un peu moins complet et moins distinct.

\section{Le dessous de l'aile antérieure.}

Tandis que le dessus de l'aile de devant a un dessin plus marqué que celui de l'aile de derrière, quant au dessous nous avons le cas inverse. L'aile antérieure est terne, excepté la marge antérieure, qui a le même caractère que sur la face supérieure, peut-être encore plus distinct.

Le dessin de la partie distale du dessous ressemble jusque dans les détails les plus minutieux à celui du dessus, excepté quant à la netteté des contours et à la clarté de la teinte. On peut constater facilement cette ressemblance en observant le lépidoptère contre la lumière.

La marge antérieure posside aussi des taches avec des centres colorés plus clairs; ces taches sont ici un peu plus distinctes qu'iu dessus; la marge postérieure porte l'ornementation des traits transversaux. La partie proximale de l'alle est unicolore, le dessin se développe de plus en plus du dedans au dehors.

Les mêmes lignes se rencontrent ici.

\section{Ite dessons de l'aile postérieure.}

Ici c'est surtout la marge costale qui est ornée de traits transversaux, marqués aussi distinetement que ceux de l'aile antérieure, prévalant même quelquefois encore en régularité. Du reste le dessin est ici plus net qu'à la face inverse, parce que la couleur de fond est la même teinte grisâtre que celle du dessus de l'aile de devant.

2. Cossus palmaris.

$$
\text { (Pl. I, fig. S). }
$$

Équateur.

Collection de Staudinger.

Le dessus de l'aile antérieure.

La couleur principale est blanche; l'ornement est brun foncé. 
Le motif réticulaire prédomine et est tracé avec une grande perfection à la partie distale de l'aile le long du bord externe.

Les mailles du lacis sont assez petites; quelquefois elles occupent la moitié, quelquefois le tiers ou le quart d'un espace internervural; il n'y a pas de régularité sous ce rapport. Nous rencontrons le méme motif le long du bord postérieur, quoiqu'on puisse observer ici une tendance à la formation de barres transversales. Le dessin produit une impression toute différente de celui de l'autre partie de l'aile, parce que les écailles ne sont pas couchées à plat, mais se dressent un peu, de sorte que le dessin a un aspect embrouillé (fig. 1).

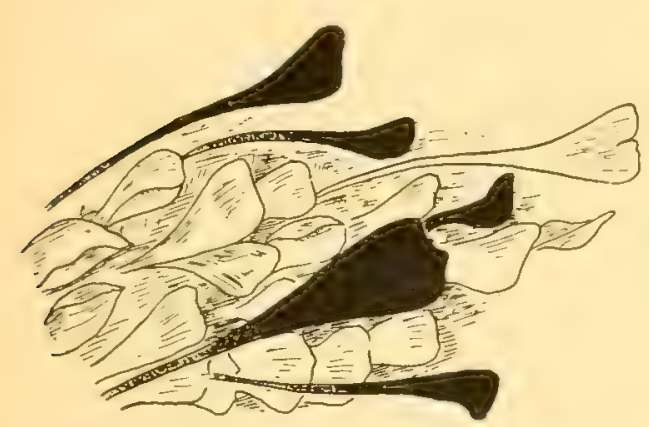

Fig. 1. Quelques écailles de Cossus palmaris entre $A n_{1}$ et $A n_{2}$.

Dans la partie proximale le dessin est souvent vague ou quelquefois presque entièrement absent. Dans d'autres endroits les lignes du réseau coloré se sont tellement élargies qu'il n'est rien resté de l'espace des mailles; de cette manière se forment de larges parties brunes dont quelques-unes constituent une bande plus ou moins continue et pour la plus grande partie transversale; la bande change de direction près du sommet de l'aile. Ses contours sont irréguliers. Elle est bornée irrégulierement. Les bandes des deux ailes ne sont pas tout à fait semblables; de petites différences se produisent; nous trouvons, par exemple, sur l'une des ailes des mailles situées a côté de la bande, tandis que sur l'autre aile les mailles correspondantes y sont incorporées.

Cette bande s'étend parallèlement au bord externe et se compose ici de fragments entre les nervures, se courbe ensuite vers la racine et en avant, pour se prolonger parallelement aux nervures longitudinales.

Deux branches ragues s'en écartent en avant et en arrière. 
Dans la cellule $R_{3}-R_{4}$ (fig. 2 ) nous voyons une tache particulière en forme de poutrelle transversale, qui est assez irrégulière et en rapport évident avec le réseau du dessin.

Tandis qu'une partie du réseau s'est noircie pour former la poutrelle, le reste s'est à peu près etfacé; comme la figure l'indique, la poutrelle montre encore très distinctement son origine par les petites dentelures et les traits transsersaux qui ornent son contour.

La marge antérieure a une rangée de barres transversales foncées sur un fond blane, qui montrent une alternation régulière

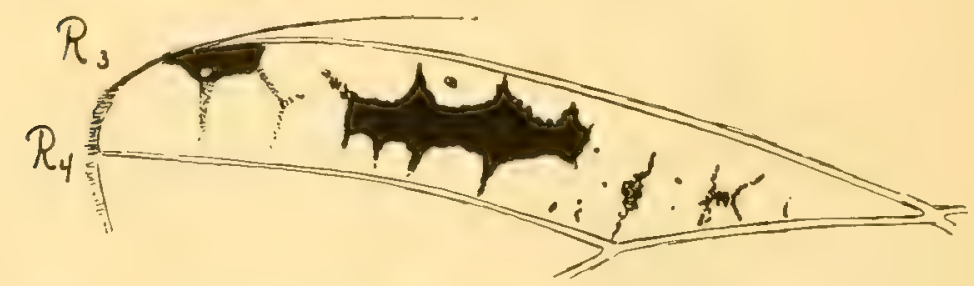

Fig. 2. Cellule $R_{3}-R_{4}$ de Cossus palmaris. Dessus de l'aile antérieure gauche.

de larges et d'étroites. On peut observer que les barres larges se composent d'un certain nombre de stries plus fines.

Les taches marginales nervurales sont très distinctes.

\section{Le dessus de l'aile postérieure.}

Est semblable ì celui de l'aile antérieure, mais brunie sur une grande partie de la surface. Evidemment ce brunissement n'est pas la conséquence d'uu simple élargissement des mailles du réseau, les espaces enfermés aussi se sont assombris en plusieurs nuances, de sorte qu'on peut rencontrer toutes les teintes intermédiaires entre la couleur blanche que nous trouvons dans les mailles de la marge externe et le brun qui occupe la partie moyenne de laile, et qui se présente comme une large bande, depuis la partie en éventail jusqu'au sommet de l'aile.

Cette partie en érentail est aussi colorée en brun.

$\mathrm{La}$ région, le long du bord antérieur, est blanche et ornée seulement sur la marge de traits caractéristiques. 
Lies dessous des deux ailes.

Les dessous possèdent le même dessin que les dessus; les différences les plus remarquables sont causées par la disposition ct la forme des écailles.

Sur l'aile antérieure les écailles de la partie moyenne sont remplacées par des poils. La même chose, mais à un moindre degré, se retrouve dans la partie analogue de l'aile de derriere.

Les marges antérieures de l’aile de devant comme de l'aile de derriere ont un dessin bien marqué et les taches marginales nervurales sont très grandes et bien limitées, surtout dans l'aile postérieure.

Si l'on place le lépidoptère devant la lumière, on voit que les dessins des dessous et des dessus se couvrent completement, aussi irréguliers qüils puissent être. Limage de chaque barre se réfléchit de l'autre côté.

Une particularité de ce Cossus sautant aux yeux, est la disposition variable des écailles sur les différentes parties de la surface ailaire; et parce qu'elle change le caractire du dessin, nous derons en dire quelques mots.

Dans la partie distale de l'aile antérieure (dessus), les écailles sont placées comme on le trouve ordinairement chez les lépidopteres, d'une maniere imbripuéc comme les tuiles d'ardoise sur un toit; ces écailles sont ovales, oviformes ou presque circulaires, et deviennent plus étroites dans la direction du bord de l'aile. Mais dans la direction vers la racine nous voyons se manifester d'antres écailles, qui sont plus grandes, longuement pétiolées et cunéiformes avec leur bord supérieur souvent tronqué en ligne droite ou échancrée (fig. 1). Elles ne sont pas couchées à plat, mais se tiennent plus ou moins debout et quelque peu pêle-mêle. Quelyues-unes s'élivent en haut sur les autres. Quelques écailles brun-fumce assez étroites et purvues d'une longue pétiole, produiscut une impression particuliore parmi les blanches et cunóiformes. Press de la racine elles prennent de plus en plus la forme de poils, quoique la forme en coin soit encore évidente.

Nous pouvons done distinguer trois types quant à leur forme et ì leur position. 
$a$ : Ecailles imbriquées;

b: Écailles plus grandes, plus larges et plus cmbronillées, se tenant plus ou moins debout;

$c$ : Écailles en forme de poil.

L'oeil nu est déjà en état de distinguer les trois types.

La partie ailaire, munie d'écailles comme sub a se produit comme une surface plane; la partie couverte des écailles nommées sub $b$, présente un aspect hérissé; on peut reconnaître le troisième type à son luisant soyeux caractéristique.

Dans l'aile postérienre le deuxiène type n'est pas reprénenté; la position et la forme des écailles est ici comme ì l'ordinaire et seulement la partie en éventail possède le type $c$.

Le dessous de l'aile antérieure est couvert, dans sa partie moyenne, d'écailles en forme de poil. Dans la direction du bord externe nous remarquons quelques-unes du type $b$, et plus rapproché de ce bord l'implantation imbriquée ordinaire.

Ca et là on voit entre les écailles susdites quelques-unes d'une forme beancoup plus différente; clles aboutissent en quelques pointes fines et doivent itre considérées probablement comme des écailles odorifères.

\section{Cossus sp. \\ (Pl. I, fig. 9).}

Collection de STAUDINGER.

Le dessus de l'aile antérieure.

La couleur de fond est d'un gris jaunâtre, celle du dessin d'un brun terne. La différence entre les deux teintes n'est pas si graiule que dans les cas ordinaires.

Ia marge antérieure possede des traits transversaux, assez distincts, qui ne dépassent pas la sous-costale.

On rencontre le mêne motif dans les cellules voisines, mais la plus grande partie de la surface ailaire est occupée du réseau coloré. Ce réseau présente beaucoup de transformations; quelquefois une partie s'est assombrie et les figures obscures, formées de cette manière dans des cellules différentes, s'unissent pour former une 
bande incomplite, entrecoupéc par les nervures longitudinales. Cotte bande incomplete se prolonge non loin du bord externe et parallèlement à celui-ci. La continuation est une sorte de bande longitudinale et irrégulière qui s'étend dans la direction de la racine. Dans cette dernière figure la couleur de foud s'est rapprochée plus de celle du dessin qui est encore assez distinct.

Nous rencontrons le long de cette bande quelques taches plus claires, partics du réseau, apparemment, dans lesquelles le dessin est plus ou moins oblitéré.

La marge postérieure aussi est ornée du motif réticulé; pourtant les éléments transversaux prédominent.

\section{Le dessus de l'aile postérieure.}

Celui-ci est d'une teinte plus terne que le dessus de l'aile de devant; dans quelques cellules les éléments du réseau se sont élargis et les parties avec la couleur de fond ont disparu à peu près.

La partie en éventail n'est pas ornée.

Comme dans l'aile de devant il y a quelques parties claires, remplies du motif réticulé.

\section{Le dessons de l'aile antérieure.}

- La marge antérieure est dessinée de traits. La région distale est ornée distinctement et présente le même dessin que le dessus. Daus la région proximale les écailles ont été remplacées par des poils et le dessin s'est oblitéré.

\section{Le dessous de l'aile postérieure.}

Le dessin est mieux développé qu'au dessus. Dans les cellules qui sont brunies, les taches elaires sont assez nettes, elles forment souvent ce qu'on pourrait nommer: une ornementatiou nuageuse. Dans yuelques cellules non loin du bord antérieur, on observe des transitions entre les traits et le réseau. La partie en éventail, surtout le long du bord postérieur, est colorée uniformément. 


\section{Cossus sp. \\ (Pl. I, fig. 10).}

Equateur. Collection de Staudinger.

Le dessus de l'aile antérieure.

La couleur de fond est blanche, le dessin brun. Dans le dernier nous retrouvons d'abord les traits internervuraux, mais en petit nombre peu rapprochés l'un de l'autre et en toutes sortes de modifications de teinte; les uns sont droits, les autres arqués; les uns se rattachent avec leurs bouts aux nervures, les autres n'y parviennent pas.

Quelques-uns de ces traits se sont élargis au milieu et devenus plus étroits aux bouts; ils sont done souvent quelque peu plus courts que les traits communs et tout à fait libres des nerrures.

De cette maniire ils prennent la forme de taclies comme nous les trouverons plus tard chez Zeuzera pyrina.

Sur l'aile antérieure s'étend, comme chez rosists palmaris, une bande irrégulière longitudinale partant à peu près de la racine et se prolongeant jusqu'au bord externe. Les bords de cette bande ne sont pas éganx, mais pourvus de dents des deux côtés, les restes évidemment des traits interuervuraux dont elle s'est déxeloppée. Les traits se sont élargis, saus doute, jusqu'à ce qu'ils se soient fondus l'un dans l'autre en formant la bande brune. On peut remarquer cela trìs distinetement sur l'aile postérieure, où se trouve une bande analogue, inais l'origine en peut être aussi assez bien distingué sur l'aile antérieure.

Il est bien curieux que les taches de la marge antérieure, des bouts des nervures et sur la marge postérieure aient l'air bien différent des autres taches: phénomìne que nous rencontrons de nouveau chez Zeuzera pyrina et qu'on trouve aussi chez les Hépialides, où Vax Bemplex y a appelé l'attention. Les taches marginales sont d'un coloris plus foncé, elles tirent au noir.

Les taches de la marge antérieure ne sont pas toutes de la même forme; surtout, quant aux dimensions, il y a des différences importantes. 
On pent remarquer facilement que les taches les plus grandes ont pris origine de la fusion de plusieurs plus petites.

Partant de la racine, la marge antérieure est ornée dans l'exemplaire examiné des parties claires et foncées suivantes:

$1^{0}$ une partie courte et claire;

$2^{0}$ une tache, longue et foncée renfermant ici et là une partie plus claire, ce qui révèle encore son origine multiple;

$3^{0}$ une partie blanche avec $2-5$ traits courts en forme de points;

$4^{0}$ une tache foncée, composée de plusieurs traits;

$5^{0}$ une partie blanche aree quelyues taches plus ou moins gramles.

La marge postérieure porte un nombre de raies assez courtes qui sont placées l'une près de l'autre.

\section{Le dessus de l'aile postérieure.}

Le dessin montre quant aux points principaux une grande ressemblance avee celui de l'aile antérieure; en détails les deux dessins different. Le premier est plus vague sauf les taches terminales, qui sont aussi foncées que sur l'aile antérieure. La bande longitudinale qui est assez large, paraît avoir tiré son origine dans cette aile de traits transversaux qui se sont élargis; nous trouvons au moins au bout de la bande des figures plus ou moins indépendantes et qui ont l'air de traits élargis, tandis que les espaces, délimités par ces figures, ont une couleur plus foncée que le reste de l'aile. Par conséquent ils produisent un enchaînement des éléments. Tandis qu'il y a dans nombre de détails de petites différences entre les deux ailes, les dessins des dessus et des dessous (sans faire attention à la plus ou moins grande clarté), sont parfaitement les mêmes; excepté là, où les écailles ont fait place aux poils; dans de tels endroits les figures du dessin ne sont présentes que du côté de la face écaillouse. C'est pourquoi l'on peut remarquer sur la face supérieure quelques tarhes dans le champ, basilaire entre la cubitale et l'anale, qu'on ne retrouve pas sur la face inférieure.

Quant it ses autres particularités l'aile postérieure possède une tache pigmentée à la marge antérieure dans sa partie distale et 
plus avant dans la direction de la racine une partie sans dessin.

La partie en éventail est colorée d'un brun égal; la frange est blanche.

Le dessous.

La surface inférieuie présente un dessin plus vague, surtout dans la partie prìs de la racine, où les écailles sont en partie remplacécs par des poils.

\section{Cossus terebra F.}

Collection de Kallenbach.

Le dessus de l'aile antérieure.

Le dessin a beaucoup de ressemblance avec celui de Cossus cossus; la couleur est d'un gris plus pur.

Le motif prineipal est celui des traits transversaux avec toutes leur's différentes modifications: bifureation, jonction, formation de lignes transversiles, affaiblissement et assombrissement ì tour de rôle et aussi formation du réseau coloré. En général nous trouvons ici des traits plus droits que chez Cossus cossus.

Le dessus de l'aile posterieure.

Celui-ci est gris avec très peu d'ornementation.

Le dessous de l'aile antérienre.

La marge antérieure est ornementée distinctement. Dans la partie distale le dessin correspond arec celui du dessus, quoique moins distinct.

\section{Le dessous de l'aile postérieure.}

Le dessin est un peu plus distinct que celui du dessus; principalement le motif réticulé y apparaît.

\section{Cossus modestus Stgr.}

Collection de Kallenbacir.

Le dessus de l'aile antérieure.

Grisâtre avec des traits transversaux, dont quelques-uns sont 
plus distincts, d'antres marqués plus fuiblement. Quelques bandes peuvent être observées. Au milieu se trouve une figure d'une forme capricieuse composée de fragments de bandes et de traits transversaux qui se sont joints d'une manière particulière.

Non loin du bord externe se prolonge une bande qui est double dans sa partie antérieure; comme quelques-uns des fragments internervuraux sont un peu arqués, des figures en forme de ( ) se sont produites.

\section{Le dessus de l'aile postérieure.}

Celui-ci est d'un blanchâtre monotone.

\section{Les dessons des deux ailes.}

Le dessous possède peu d'ornementation; les marges antérieures ont des écailles grises et brunes entremêlées.

\section{Leuzera pyrina L.}

Europe. Six exemplaires dont quatre dans la collection de KatLenBaCH.

Le dessus de l'aile antérierre.

La couleur de fond est blanche; le dessin consiste en rangées de taches dans les espaces internervuraux; ces taches sont noires avec un éclat métallique. Dans chaque cellule il y a seulement une rangée, ainsi que dans celles du champ discoildal. Ces taches sont ordinairement ovales ou rondes, quelquefois un peu rétrécies, mais toujours à bouts arrondis; elles n'atteignent pas les nervures longitudinales. Ca et là on distingue la jonction de deux taches; une tache en forme de $\vee, \wedge$ ou $X$ prend alors naissance. Elles sont rangées en général de telle manière que leur axe long est perpendiculaire aux nervures longitudinales; mais l'axe de quelques-unes est placé un peu obliquement. La teinte de toutes ces taches n'est pas toujours la même; dans un exemplaire les taches ont un coloris foncé, dans un autre le coloris semble affaibli. Il est curieux que dans ces derniers exemplaires les taches marginales conservent leur pigment, elles sont différen- 
ciées quelquefois encore plus; dans un des exemplaires quatre taches de la marge postérieure s'étaient fondues; l'origine quadruple était encore bien visible.

Les taches de la cellule costale s'unissent quelquefois avec celles des cellules adjacentes; celles de la marge externe sont intéressantes, en ce point qu'elles sont les seules qui soient situées sur les nervures longitudinales.

Autour de la nerrure discoïdale s'étend un champ blanc; cette nervure n'est pas colorée dans la majorité des cas; mais dans un senl exemplaire je remarquais ici une petite bare noire au dessus. Une, telle absence presque complète de l'ornementation de cette nervure est sans doute remarquable, parce que chez la plupart des Lépidoptères elle appartient aux ornements les plus constants et même se produit quand les autres décorations sont tout it fait absentes.

Le nombre des taches n'est pas le même pour les différents exemplaires, quand on compare des cellules analogues, quoique les limites entre lesquelles les nombres se meurent, ne soient pas très éloignées les unes des autres. On peut trouver les mêmes variations quant à la forme.

\section{Le dessus de l'aile postérienre.}

Celui-ci possède un dessin analogue à l'aile antérieure.

Quelquefois, mais pas toujours, les cellules analogues ont le même nombre de taches.

Ordinairement elles sont plus pâles; exceptées celles de la marge antérieure.

La partie en éventail est toute blanchâtre; une trace de dessin peut être quelquefois observée.

\section{Les dessous des deux ailes.}

Les dessous ont le même dessin que les dessus.

Dans ce Lépidoptère je pouvais rechercher facilement la cause de la pâleur des taches. Dans les taches foncées (fig. 3) Jes ćcailles sont serrées; plus la tache est pâle, plus les écailles sont 
clair-semées. Non seulement celles-ci sont placées plus loin l'une de l'autre, mais elle sont aussi plus étroites (fig. 4); par ces deux causes la membrane ailaire devient visible.

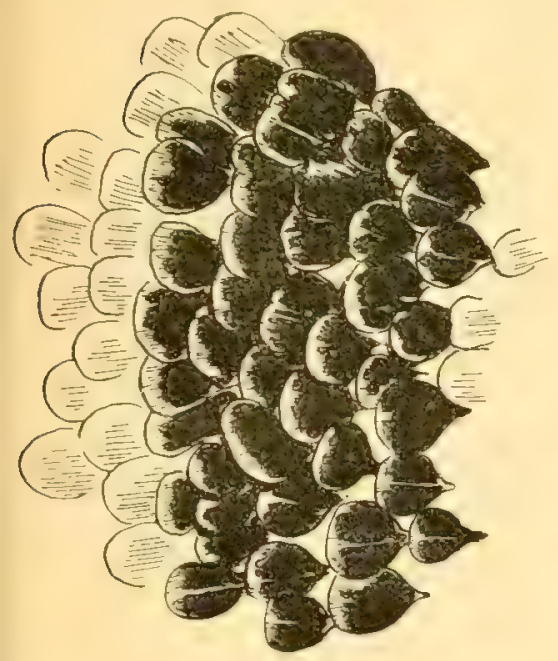

Fig. 3.

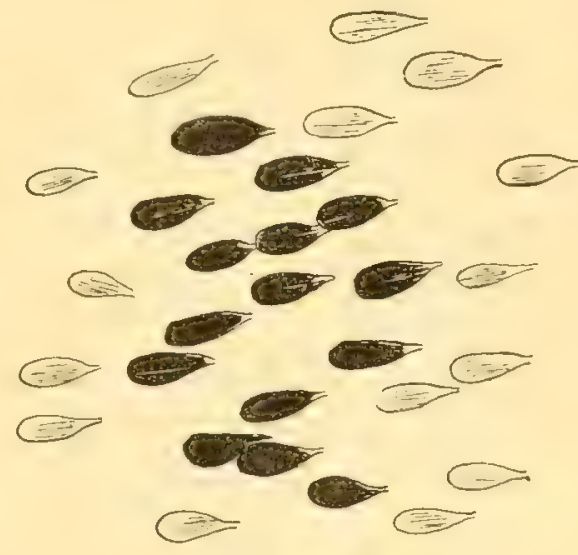

Fig. 4.

Une des taches foncées de Zeuzera pyrina. Une des taches pûles de Zeuzera pyrina.

Sur les ailes dont les taches noires sont devenues plus pâles, les écailles blanches ont diminué également en nombre et en largeur.

\section{Zeuzera sp. \\ (Pl. II, fig. 2).}

Bornéo. Collection du Jardin Zoologique d'Amsterdam.

Ce Lépidoptère a la même forme de Zeuzera pyrina mais le dessin, quoique composé de taches, est tout autre.

\section{Le dessus de l'aile antérienre.}

Dans la marge antérieure nous rencontrons 9 ou 10 taches it peu près rondes qui ne sont pas développées toutes au même degré. Une tache non loin du sommet dépasse quelques nervures. Une rangée de quatre s'étend sur la surface ailaire commençant près du sommet. 
Ces taches ne sont pas situées entre mais sur les nervures, qu'elles dépassent des deux côtés.

Les nervures ornées de cette manière sont $\mathrm{M}_{1}, \mathrm{M}_{2}, \mathrm{M}_{3}$ et $\mathrm{Cu}_{1}$; la rangée est un peu arquée avec la convexité en dehors. Sur $\mathrm{Cu}_{2}$ il y a aussi une tache, rapprochée du point de bifurcation. Dans le champ discoildal on en rencontre 3 ou 4 ì dimensions différentes, qui dépassent aussi les nervures. Sauf les taches indiquées et les taches terminales, toutes les autres sont internervurales. Entre le $\mathrm{Cu}_{2}$ et $\mathrm{l}^{\prime} \mathrm{An}_{1}$ on en remarque 4 ou 5 , dont 2 ou 3 ont la forme de traits transfersaux; les deux autres sont situées non loin de la racine.

Dans la cellule adjacente on peut encore en compter 5 et le long du bord postérieur encore 2 taches qui sont allongées. Les taches marginales nervurales sont bien développées. Toutes les taches sont noires avec ua éclat de pourpre.

Le dessus de l'aile postérieure.

Pas de taches, excepté au bout de quelquiss nervures longitudinales.

Les dessous des deux ailes.

Les dessous sont dessinés comme les dessus.

9. Duomites leuconotus Walker.

Ceylan. Collection de Staudinger.

Le dessus de l'aile antérieure.

La couleur de fond est gris brunâtre, exceptéo la pointe, qui est blanche comme la marge postérieure et la moitié postérieure de la marge externe. Cette marge a une limite intérieure capricieuse qui commence sur $\mathrm{M}_{3}$, dépasse $\mathrm{Cu}_{2}$ et ensuite se courbe dans la direction vers la racine, pour atteindre rapidement le cubitus de sorte qu'uue mince partie du champ brun est située derrière cette nervure; puis la limite court dans une direction oblique en arrière presque jusqu'à $\mathrm{An}_{2}$ pour, enfin, se diriger obliquement en arant jusqu'ì la racine de la radiale. La limite est formée par des éléments foncés du dessin. 
Une tache claire, mais indistinctement délimitée, est située dans le voisinage de la bifurcation de $R_{3}$ et $R_{4}+{ }_{5}$; on en reneontre une autre à la fin du champ discoïdal.

La marge antérieure a pour dessin un certain nombre de traits foncés qui se suivent sans beaucoup de régularité. Sur la partie restante le dessin se compre principalement de traits trausversaux qui se tiennent nettement dans le cours des nervures.

Quelques-uns sont droits, la plupart sont courbés ou tortueux, élargis au milieu ou anastomosant. Eu dehors du champ discoïdal il y a entre $\mathrm{H}_{1}$ et $\mathrm{M}_{2}$ une tache noire, longitudinale et dentelée, apparemment composée de traits transversaux.

Dans la marge externe nous trouvons un réseau coloré de mailles irrégulières qui deviennent moins distinctes en arrière. En $\mathbf{R}_{5}-\mathbf{M}_{1}$ les lignes du réseau s'élargissent en forme de tache. Nous remarquons un eas analogue dans quelyues traits du champ discoülal.

Dans la marge postérieure il y a des traits qui se ramifient et s'anastomosent.

Les taches marginales nervurales peuvent être nettement distinguées; de même celles qui sont situées dans la partie blanche.

\section{Le dessus de l'aile postérieure.}

Celui-ei est d'un brun unicolore, aree une seule tache blanche it la marge externe non loin de la quene de l'aile. Le motif des traits domine également ici; quelques-uns d'entre eux ont la forme de sablier; en quelques endroits un réseau coloré se produit. En gévéral la teinte des éléments du dessin est plus terne et c'en est le même arec lia couleur de fond. La partie en érentail est incolore.

Dans Seitz (69) il y a une image d'un exemplaire de ce Lépidoptère qui a beaucoup de ressemblance avec le mien; la partie blanche de la marge externe s'étend un peu plus en avant et les taches claires sont plus grandes et plus distinctes.

\section{Le dessous de l'aile antrienre.}

La marge antérieure est dessinée plus nettement qu'au dessus; 
la conleur de fond est la même qu'à la face supérieure; la marge blanche externe ne se prolonge pas si loin en avant et n'accompagne pas tout le bord postérieur.

(Une partie de la marge postéricure est invisible, couverte par l'aile de devant).

La surface ailaire inférieure possède la même ornementation que la face supérieure, mais moins distincte.

Le dessous de l'aile postérieure.

Apparemment la marge antérieure est d'une couleur uniforme; mais en réalité elle a les mêmes figures qu’au dessus.

\section{Xyleutes myracmon Cram. 0 .}

Équateur. Collection de Staddinger.

Le dessus de l'aile antérieure.

La marge antérieure présente:

$a$, une grande tache, brune, longitudinale;

$b$, une partie blanche avec environ cinq points foncés;

$c$, une tache noire, un peu plus grande;

d. une partie blanche avee 3 ou 4 petites taches.

Sur l'aile s'étend, un peu en zigzag, une bande irrégulière, longitudinale, qui commence dans la cellule $R_{4}-R_{5}$ et qui, en $\mathrm{M}_{3}-\mathrm{Cu}_{1}$, prend une direction vers la racine pour se courber un peu plus tard en arrière. La bande trahit distinetement dans la partie distale sa cohérence avec le réseau coloré et plus intérieurement avec les traits internervuraux.

Nous remarquons ces éléments encore attachés aux côtés de la bande.

Cette bande approche la tache brune sub $a$, mais en est séparée par un espace aree une petite tache et quelques traits.

Devant la nervure anale il y a une tache qui a tiré évidemment son origine du réseau.

Les autres parties de la surface sont ornées de traits bruns ou des fragments de réseau coloré; les derniers dans la partie distale, les premiers danś la partie proximale. Entre ces deux motifs il 
y a de belles figures intermédiaires; on les voit, par exemple, dans la cellule $\mathrm{Cu}_{2}-\mathrm{An}_{1}$ dans l'aile gauche; d'abord on $\mathrm{y}$ roit un certain nombre de barres droites, puis quelques-unes plus sinueuses avec des anastomoses et ensuite le motif réticulé. On renentre les memes formes intermídiares dans la cellule $R_{5}-M_{1}$.

La couleur de fond est blanchâtre.

Les taches marginales nervurales sont présentes, mais pas très nettes, ce qui pourrait être la suite du maurais état de mon exemplaire. La tache de la seconde nervure anale $\left(\mathrm{Au}_{2}\right)$ est encore la plus visible.

Le dessus de l'aile postérieure.

Celui-ci a beaucoup de ressemblance avec le dessus de l'aile de devant; la bande longitudinale est plus claire et moins distincte; raison pour laquelle la cohérence avec les motifs simples du dessin n'est pas sculement visible aux côtés, mais aussi ì l'intérieur. Lit partic en évent:il est partiellement claire, partiellement sombre; la marge antérieure est colorée blanche, mais possède encore des traits distincts.

Le dessous de l'aile antérienre.

Lo même dessin que le dessus; les éléments des deux faces se couvrent parfaitement. Dans la partie moyenne le dessin est moins net. La marge antérieure est ornée arec la même netteté que celle du dessus. Une des taches démontre son origine de traits transversax. Lat marge postérjeure al une ornementation moins nette.

Le dessons de l'aile postérieure.

L'ornement de la marge antérieure est bien déreloppé; un trait noir, large en forme de coup de pinceau longe la sous-costale; les traits transversaux sont très typiques dans la cellule $\mathbf{M}_{1}-\mathbf{I I}_{2}$ de l'aile droite.

$$
\begin{aligned}
& \text { 11. Xylentes sp. }{ }^{\text {. }} \\
& \text { (PI. II, fig. } 5 \text { et } 6) \text {. }
\end{aligned}
$$

Océanie. Collection du Jardin Zoologique d'Amsterdam.

Cette espèce semble être identique arec Tyleutes d'urvillei, en 
jugeant d'après les images de Herricil-Scinaffer (30, fig. 162-164).

\section{Le dessus de l'aile antérieure.}

Les nervures, ou mieux, les écailles qui couvrent les nervures, sont colorées brun de cuir. Près de la racine la cellule costale est élargie, parce que le bord antérieur est arqué en avant. Les traits transversaux foncés qui sont placés à des distances assez régulières dans cette partie élargie, sont un peu ramifiés et anastomosant. Les traits dans les autres cellules se tiennent nettement daus le cours des nervures et les cellules $\mathbf{S C}-\mathbf{R}_{1}$ et $\mathbf{R}_{2}-\mathbf{R}_{3}$ montrent que les figures d'une certaine cellule n'ont pas de rapport avec celles d'uue edlule adjacente. Dans les parties distales de $R_{2}-R_{3}$ et $R_{3}-R_{4}$ on voit les traits transversaux se modifier en réseau.

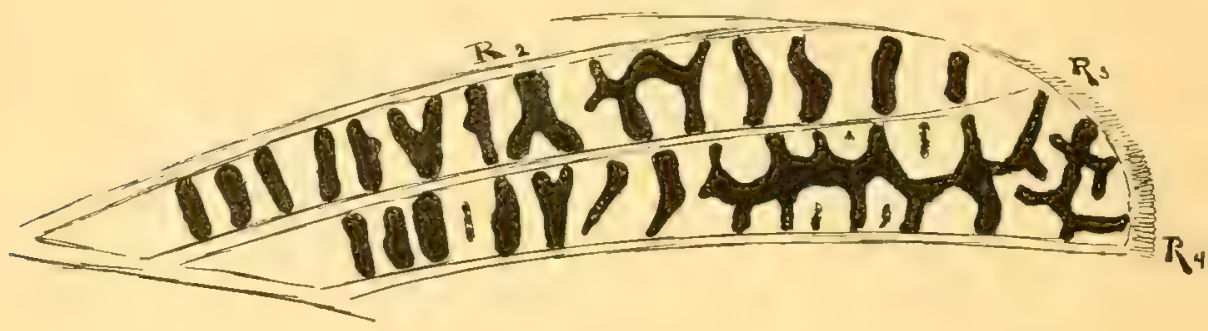

Fig. 5.

Fragment de l'aile antérieure de Iyleutes sp. (d'urvillei'?) Q. Transitions des traits en réseau coloré.

Dans la cellule $R_{2}-R_{3}$ ectte transformation a lien de la manière suivante (fig. 5). D'abord on remarque un certain nombre de traits non ramifiés, puis un en forme de $V$; le trait suivant est simple; suivent alors deux figures dont l'une a la forme de la lettre $\mathrm{Y}$ arec l'ouverture en bas et l'autre est formée de quelques traits avec des jonctions; dans la partie la plus distale on voit de nouveau quelques traits simples.

Dans la cellule adjacente le réseau se complète un peu plus; nous voyons ici deux rangées de mailles, tandis que la ligne de délimitation de ces deux rangées est un peu élargie ct devenue 
plus pigmentée. Dans les espaces des mailles nous trouvons quelquefois un trait raccourci ou un centre plus clair que le réseau, mais plus foncé que le reste de l'espace enfermé de la maille.

Au bord externe la régularité disparaît.

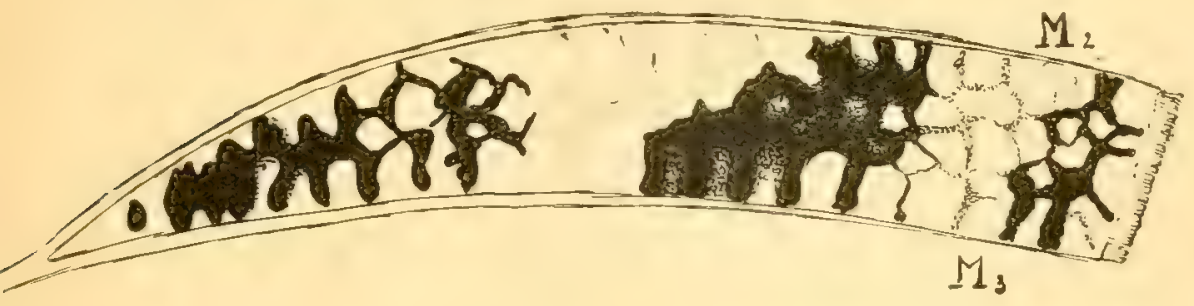

Fig. 6 .

Fragment de l'aile antérieure de Iyleutes sp. (d'urvillei i) ㅇ.

Dans la cellule $\mathrm{I}_{2}-\mathrm{II}_{3}$ (fig. 6) nous remarquous déjà du premier coup d'œil que le nombre des mailles a augmenté. Entre la cubitale et la nervure $\mathrm{An}_{1}$ le réseau est remplacé par le motif des traits. La même transformation se produit entre $\mathrm{An}_{1}$ et $\mathrm{An} \alpha$.

De la pointe de l'aile une bande se prolonge dans une direction transversale qui diverge un peu aree le bord externe et qui se compose d'autant de fragments, qu'elle parcourt d'espaces internervuraux. Ces composants de la baude sont des fragments du réseau coloré, dont les mailles sont devenues plus larges aux dépens de l'espace enfermé, tandis que le dernier a pris souvent un coloris plus foncé (fig. 6).

Les divers fragments ne sont pas situés dans le prolongement l'un de l'autre, mais font un angle entre eux.

Une bande semblable, mais sautant moins aux yeux, parce que les lignes du réseau ne se sont que peu élargies, s'étend du côté extérieur de la première bande transversale. On peut remarquer un fragment de ceite bande dans notre figure. Le réseau entre ces deux bandes et en dedans de la bande interieure s'est affaibli ct a disparu à peu près complètement en quelques endroits.

D'autres parties pigmentées se trouvent dans la région moyenne de l'aile. Nous en rencontrons une dans la partie proximale de la cellule $\mathrm{An}_{1}-\mathrm{An} \propto$ qui est reliée à une tache entre $\mathrm{An}_{1}$ et $\mathrm{Cu}_{2}$. 
Cette partie longe la nervure $\mathrm{Cu}$; derrière cette partie allongée et pigmentée nous trouvons dans la mime cellule le rísean colorí. Cette partie foncée peut être considérée comme la continuation de la bande transversale. Un autre noircissement se produit encore en dehors du champ discoildal en forme de figures longitudinales qui constituent ensemble une sorte de bande large et transversale. Dans le ehamp discoïdal les nervures sont colorées en brun et chaque cellule a sa striation internervurale indépendante.

La marge postérieure est dessinée d'une manière particulière. Nous rencontrons ici des barres longues, noires, serrées et simueuses qui ne se ramifient qu'un peu et qui ont des jonctions entre elles. Elles forment sur le fond jaune et blanc ce qu'on pourrait nommer: un dessin tigré.

Dans le voisinage de la racine on peut déduire du dessin la présence de la nervure $A_{n} \beta$, qui n'est pas visible.

\section{Le dessus de l'aile postérieure.}

Les écailles blanches manquent ici. Le noir et le brun de l'aile de devant sont plus ternes. Le motif est celui du réseau réticulé. Quelquefois on ne rencontre une ornementation qu'au milieu des espaces internervuraux.

La partie en éventail est uniformément brune, mais possède encore une trace de dessin.

La marge antérieure est colorée elaire et est devenue en partie blanche. A l'endroit où la partie couverte de l'autre aile touche ì la partie non-couverte, nous pouvons observer quelquos traits transversaux.

\section{Le dessous de l'aile antérieure.}

Une grande partie de l'aile est incolore, savoir: la partie qui est pourvice d'icililles piliformes; les marges seulement montrent un dessin distinct. La marge antérieure porte des barres transversales uettes ot rémglieres, qui se comportent it la partie ćlargie de la même manière qu'au dessus, seulement un noireissement se produit le long de la sous-costale. 
La marge externe a les mêmes figures que celle du dessus mais plus vagues. Une partie le long du bord postérieur est blanchâtre, la marge postérieure n'est pas visible.

Le dessous de l'aile postirieure.

La marge antérieure est dessinée de traits; du reste le même dessin que le dessus; la partie en érentail est unicolore. Au milieu des espaces internervuraux nons voyons dans le réseau des taches longitudinales dentelées.

Une grande partie de cette surface est brune.

$$
\begin{aligned}
& \text { 12. Tylentes sp. } 0^{7} \text {. } \\
& \text { (Pl. II, fig. } 3 \text { et 4). }
\end{aligned}
$$

Collection du Jardin Zoologique d'Amsterdam.

L'exemplaire reproduit dans la planche II est vraisemblablement un individu mâle de l'espèce précédente.

II est plus petit, mais quant au dessin il $\mathrm{y}$ a beaucoup de ressemblance. Níanmoins il y a quelrues diffírences; par exemple, la bande oblique se perd insensiblement dans la partie distale et les espaces entre les branches de la média et de la cubitale ont moins de mailles; la continuation longitudinale de la bande est moins déreloppée et la tache qui y est jointe se compose de fragments du réseau coloré; la deuxième bande extérieure est aussi moins distincte.

$\mathrm{Au}$ dessous on voit des différences analogues; l'aile postérieure a plus de blane, mais quant au reste, elle est pareille à la surfaco supérieure.

$$
\begin{aligned}
& \text { 13. Xyleutes sp. }{ }^{1} \text { ). } \\
& \text { (Pl. II, fig. } 7 \text { et } 8 \text { ). }
\end{aligned}
$$

Océanie. Collection du Jardin Zoologique d'Amsterdam.

Le dessus de l'aile antérienre.

L'aile est blanche et brune; le blane est la couleur du fond;

1) Je regrette beaucoup de n'avoir pu constater les noms spécifiques de ces Lépidoptères si intéressants; j'espère que les images pourront dédommager un peu lo lecteur. 
le brun celle du dessin. Dans la marge antérieure nous rencontrons des barres brunes, alternant avec des espaces blancs; c'est dans la partie près de la racine que les barres sont le plus larges; ici quelques jonctions ont eu lieu. Au tiers de la distance de la racine jusqu'au sommet, partant de la racine, nous remarquons une tache qui s'est forméc apparemment de deux traits et qui est cohérente avec une bande mal délimitée, traversant la surface ailaire.

Puis on voit quelques traits qui sont tout à fait séparés l'un de l'autre dans l'aile gauche, tandis que les éléments analogues dans l'aile droite sont unis entre eux près de la sous-costale. Aux deux tiers environ du bord antérieur une autre tache se produit, évidemment le commencenent d'une autre bande très courte.

Alors suivent quelques taches irrégulières, alternant arec des traits transversaux.

Dans la marge externe l'aile est ornée d'un réseau coloré. Celui-ci forme non loin du bord une bande qui se distingue de l'autre partie du réseau par les mailles, dont les contours sont élargis et fortement pigmentés. Aux deux côtés de cette bande le réseau est beaucoup moins développé, de sorte que la bande est accompagnée de deux étroites bordures blanches. Une cellule avec un fragment de cette bande est présentée dans la figure 7.

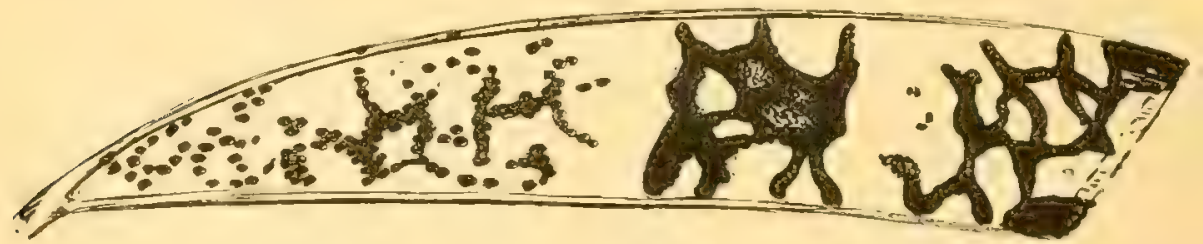

lig. 7. Cellule $\mathbf{M}_{2}-\mathrm{M}_{3}$ de l'aile antérioure do Iylentes sp. Dessus.

La bande commence au sommet et se compose de fragments, situés entre les nerrures. C'est dans la partie moyenne que ce fragment est le plus foncé; les parties longeant les nervures sont plus claires. En dehors de cette bande et de sa bordure un réseau plus net se produit qui est lui aussi le plus distinct au milieu de chaque espace internervural. 
Les taches marginales nervurales sont bien marquées et liées au réseau. Cette liaison se manifeste par la présence de mailles ¿̀ espaces assombris, dans la périphérie des taches.

La marge postérieure montre un beau dessin réticulé. Entre les nervures $\mathrm{An}_{1}$ et $\mathrm{An}_{2}$ il $\mathrm{y}$ a des traits qui ont la forme d'un sablier; c'est avec ces sabliers qu'alternent des figures élargies au milieu. De lì il résulte des figures ovales à centre foncé.

Du côté intérieur de cette bande nous rencontrons encore quelques fragments de dessin réticulé qui se dissolvent et se transforment inseusiblement dans l'ornementation dittuse de la partie unicolore du milieu de l'aile (voir fig. 7).

Unicolore n'est pas, à vrai dire, le mot, car dans cette région, il $\mathrm{y}$ a des écailles brunes et blanches qui sont fortement entremêlées, de sorte qu'il s'ensuit une couleur mélangée. Il y a beaucoup de transitions parce que les écailles brunes du dessin se dispersent graduellement entre les écailles blanches du fond. Non loin de la racine, nous trourons quelques taches mal délimitées, constituant une figure en forme de bande qui, comme nous l'avous vu en parlant do la marge antérieure, atteint cette marge au tiers de sa longueur.

On rencontre entre $A n_{\ell}$ et $A_{n}$ une autre tache, séparée de cette ,bande" par une partie blanche. C'est la continuation de la bande transversale déjà mentionnée.

Le dessus de l'aile postérieure.

Celui-ci est en grande partic d'une couleur brune uniforme; la marge externe porte un dessin brun it fond blanc; les taches marginales nervurales sont bien déreloppées.

Dans la partie unicolore nous trourons encore des traces d'un dessin réticulé foncé.

La première partie de l'aile postérieure est blanche et n'a pas de dessin pour autant que je pus l'observer.

La partic en érentail est partiellement brune, partiellement blanchâtre. 


\section{Le dessous de l'aile antérienre.}

La marge antérieure a des traits transversaux bruns. Quelquesuns se sont joints, enfermant quelquefois un centre blanc, en d'autres cas formant une tache plus grande.

Déjà entre $R_{2}$ et $R_{3}$ le dessin réticulé se manifeste.

Une marge considérable le long du bord externe possède justement le même dessin qu'au dessus. Dans la bande transversale le dessin réticulé ressort moins clairement sur le fond qu'au dessus.

Du côté intérieur de la bande nous trouvons entre $\mathrm{I}_{1}$ et $\mathrm{M}_{3}$ me partie pigmentée allongée. Une petite partie de la bande près de la racine est encore présente dans le voisinage du subcosta. La partie postérieure de l'aile est blanche avec quelques traces de dessin.

Les taches marginales nervurales sont bien développées.

\section{Le dessous de l'aile postérieure.}

La marge antérieure est couverte du motif des traits; une partir pigmenté lomgitudinale s'itend le long de la sons-costale; dans la partic distale on rencontre le motif réticulé. Il y a cncore des vestiges d'une bande; une tache près du sommet saute aux yeux. Le champ basilaire est à peu près unicolore par l'assombrissement de la couleur interne des mailles. La partie en éventail est colorée comme au dessus.

\section{Xylentes sp.}

(Pl. II, fig. 9 et 10).

Océanie. Collect. du Jard. \%ool. d'Amsterdam.

Le dessus de l'aile antérieure.

Celui-ci a une teinte uniforme; par un examen approfondi cette teinte paraît être mêlée; les écailles blanches et brunes s'y trouvent pêle-mêle. Il y a encore un vestige trè vague d'une bande; dans l'endroit où cette bande atteint le bord externe, un petit point pigmenté se produit sur la frange non loin du sommet. On peut aussi observer des traits ragues le long du bord 
postérieur, quand l'angle d'incidence des rayons lumineux atteint une certaine valeur.

\section{Le dessus de l'aile postérieme.}

Celui-ci est aussi unicolore: ronge-brun. Une partie le long du bord externe est teinté un peu plus clairement; une partie le long du bord antérieur est blanche; où cette partie se joint ì la partie rouge, les écailles brunes et blanches sont entremêlées. La région blanche est courerte de très petites écailles. Dans la partie en éventail nous rencontrons des écailles piliformes.

\section{Le dessous de l'aile antérienue.}

Les marges antérieure et externe sont colorées comme au dessus.

La bande est ici un peu plus distincte et se compose de fragments dont chacun fait un angle avec la direction de la bande. Une des dernières taches est même longitudinale: e'est la tache cntre $\mathrm{Cu}_{1}$ et $\mathrm{Cu}_{2}$; de ce point la bande se tourne vers la racine. Les autres taches montrent aussi une petite extension dans une direction longitudinale. La partie de la bande entre $\mathrm{Cu}_{1}$ et $\mathrm{An}_{2}$ est située à la moitié de la distance entre la racine et le bord exteme.

La région ultérieure do la partie en éventail est blanche et montre une ondulation légère. Une grande partie de la surface ailaire est rouge-brun avec des écailles piliformes. Dans cette partie on pent observer une tache très vague. La similitude des deux dessous est très grande et forme un contraste avec la dissimilitude des deux dessus.

\section{Le dessous de l'aile postéricure.}

La marge antérieure est d'une teinte blanchâtre qui est mêlée arec des écailles rouges. Prìs de la racine il y a une partie blanche; une large marge le long du bord externe est gris-brun; les autres parties ont un coloris brun-rouge.

Sur les nervures les poils bruns-rouges se rencontrent plus loin rers le bord externe que dans les espaces internervuraux, de sorte qu'il se forme des raies allongées nervurales. 


\section{Xyleutes lituratis Donov.}

(Pl. II, fig. 11).

Un exemplaire mâle. Collect. du Jard. Zool. d'Amsterdam. Le dessus de l'aile antérieure.

La marge antérieure porte des taches irrégulières plus ou moins grandes. Dans les cellules étroites le long du bord antérieur le motif des traits domine. Dans le champ discoïdal toutes les cellules peuvent être reconnues à leur hachure au moyen de ces figures. Dans la partie distale nous royons le motif réticulé qui a la tendance à former de grandes taches. Une de ces taches se rencontre à la fin distale du champ discoidal, une autre au bord externe, un peu derrière le sommet.

Les taches marginales, nerrurales sont bien développées.

Le long du bord postérieur on remarque un résean coloré, dont quelques parties ont disparu, de sorte qu'il se forme des figures pigmentées, ramifiées en forme d'arbre.

Le dessus de l'aile postérieure.

Celui-ci possède à peu près le même dessin, mais moins distinct, parce que la couleur de fond se rapproche de celle du dessin, surtout au milieu de la surface ailaire. La marge antérieure possède peu d'ornementation.

\section{Le dessous de l'aile antérienre.}

La marge ressort clairement sur l'autre partie, parce que la cellule entre la costale et la sous-costale est blanche arec des taches noires assez grandes.

L'ornementation, quoique plus vague que celle du dessus, y ressemble beaucoup, exceptée dans la marge postérieure, où il n'y a que de minces restiges.

Le dessous de l'aile postérieure.

La marge antérieure est couverte de traits quelque peu irréguliers. En certaines parties les traits prévalent, en d'autres le motif réticulé. Ce motif peut être constitué d'une manière particulière. 
On remarque dans un exemplaire (non-reproduit) des parties de fond claires assez grandes avec une figure ramifiée au centre.

\section{Tyleutes (Endoxyla) strix L.}

(Pl. II, fig. 12. Pl. III, fig. 1-4).

3 exemplaires dans la coll. du Jardin Zool. d'Amsterdam.

2

Le dessus de l'aile antérieme.

Dans un des exemplaires de la collection du Jardin Zool., originaire de Céram, dont l'envergure est à peu près de $18 \mathrm{~cm}$. , la marge antérieure est dessinée de taches larges. Ia prenière partie de cette marge, en partant de la racine, est colorée en brun, puis vient une partic daire avec quelques petites taches pigmentécs, puis une tache plus grande, qui paraît être construite de trois plus petites, et ensuite un certain nombre de taches plus ou moins grandes.

Les taches se maintiennent dans le cours des nervures; elles ne dépassent pas la sous-costale. On trouve dans les cellules adjacentes étroites quelques petites taches ou traits. Derrière la grande tache dans la cellule $\mathrm{C}-\mathrm{SC}$ les barres de $\mathrm{SC}-\mathrm{R}$ se sont élargies. Dans le champ discoïdal on rencontre un dessin net qui se règle conformément au cours des nervures, de sorte qu'on peut déduire la neuration de la disposition des éléments du dessin. Dans les autres parties de la surface les deux motifs des Cossides (les traits et le motif riticulé) sont partagés comme it l'ordinaire: les traits occupent la partie proximale et le réseau courre la partic distale.

Les traits sont quelquefois droits, le plus souvent arqués; souvent élargis, tantôt régulierement, tantit irrégulierement, ramifís et joints entre eux par des barres de jonction.

Le fond est gris-brun, mais possède ģa et là des régions blanches entre lesquelles le brun de fond s'enroule: en forme de bande irreguliere, évidemment comme une formation homologue de la bande irrégulière de Cossus palmaris et d'autres espèces.

On rencontre dans la partie foncée du fond des taches noires 
qui trahissent distinctement leur origine de traits joints. D'abord nous avons une série parallèle au bord externe; dès $R_{\mathfrak{z}}$ la bande se tourne en dehors et atteint ici le bord. De cette rangée une autre sort, it la hauteur de $\mathrm{C}^{\prime} \mathrm{u}_{2}$, obliquement en avant, dans liaquelle la grande tache est située à la fin du champ discoïdal.

Quelques autres se trouvent entre $\mathrm{Cu}_{2}$ et $\mathrm{An}_{2}$ et une tache dans la partic basilaire des cellules interjacentes. I a surface entre $\mathrm{An}_{2}$ et le bord postérieur possède seulement dans le voisinage de la queue de laile quelques traits transversaux, mais, pour le reste, montre peu de dessin.

Les taches marginales, nerrurales sont présentes mais presque invisibles.

\section{Le dessus de l'aile postériemre.}

Celui-ci a une couleur plus uniforme; la différence de teinte entre le fond et le dessin n'est pas si important que sur l'aile de devant. Au milieu il y a une partie pigmentée qui est entourée par des marges plus elaires.

J'ai remarqué des traits dans la marge antérieure, pour antant que j'ai pu voir celle-ci.

Les taches marginales nervurales sont présentes.

La partie en éventail est d'un brun égal.

Le dessous de l'aile antérieure.

La marge antérieure est dessinée clairement comme les cellules $\mathrm{SC}-R_{1}$ et $R_{1}-R_{2}$; le dessin est du reste plus vague, les taches ressortent moins sur le fond, mais les éléments du dessin sont parfaitement les mêmes des deux côtés. L'ornementation la plus nette est le long du bord externe.

Le dessous de l'aile postérieure.

Contrairement ì ce que nous arons vu dans l'aile de derant, le dessin est ici un peu plus clair et plus riche qu'au dessus.

La marge antérieure porte des traits, la marge externe le motif réticulé.

La cellule costale est ornée très distinctement de traits trans- 
versaux, tantôt simples, tantôt transformés dans l'une ou l'autre direction; direction qui n'est pas toujours parfaitement la meme sur les deux ailes d'un individu. (Fig. 8 et 9).

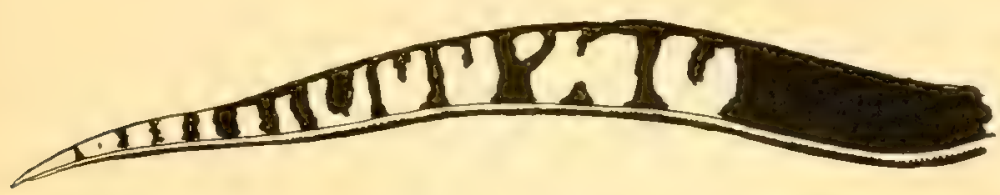

Fig. 8. Cellule costale (C-SC) de l'aile postérieure drcite (dessous) de Iyleutes strix 오.

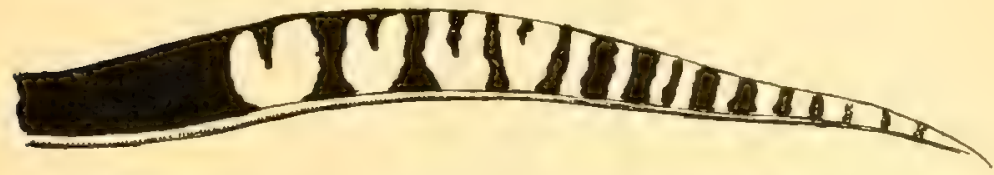

Fig. 9. Cellule costale (C-SC) de l'ailo postérieure gauche (dessous) de Tyleutes strix 우

La région ultérieure de la partie en éventail, par conséquent le long $\mathrm{du}$ bord postérieur, porte aussi des vestiges de figures.

Sur les deux ailes, aussi bien sur le dessus que sur le dessous, il y a un éclat violacé, qu'on peut observer sous un certain angle d'incidence des rayons lumineux.

Deux autres femelles présentent de grandes différences entre elles et avec la forme décrite plus haut. Tandis que dans l'une les traits forment le motif dominant, on rencontre dans l'autre sur la plus grande partie de la surface ailaire le réseau coloré. La dernière femelle a en ontre bencoup plus de hanc; les taches blanche's se sont étendues vraisemblablement au dépens du fond brun clair. Ce fond aussi est d'un coloris plus clair que dans lexemplaire décrit. Dans les parties blanches mentionnées le dessin n'est pas très complet; dans les parties plus foncées les taches pigmentées se sont moins développées. Le réseau de l'exemplaire clair montre des mailles de différente's dimensions; il y en a de plus grandes et de plus petites, qui sont grouperes irrégulierement; dans les parties blanches le réseau est souvent rompu ça et là et les contours sont fréquemment devenus plus étroits et d'un coloris plus faible. 
Quelques taches sont encore assez distinctes:

$1^{0}$ une entre $R_{4}$ et $M_{2}$, qui trahit nettement son origine du réseau par la présence de lignes un peu plus foncées, enfermant des petits espaces plus clairs.

2" une à la fin du champ discoïdal, cohérente au réseau, comme la précédente.

$3^{0}$ une entre $\mathrm{Cu}_{2}$ et $\mathrm{An}_{2}$.

Les taches marginales sont grandes et bien développées.

La marge postérieure possède, contrairement aux deux autres exemplaires, un dessin réticulé complet, dans lequel pourtant les lignes transversales dominent.

Le dessous n'est orné qu'aux marges.

On trouve aussi des figures le long du bord anal de l'aile postérieure.

Le deuxième exemplaire de la collection de Staudinger s'est développée dans une autre direction.

Ce sont ici surtout les traits transversaux qui composent le dessin (Pl. II, fig. 1 et 2). Ces traits se sont quelquefois élargis au milieu, où il y a ça et là une jonction, de sorte qu'on peut constater ici le début d'un réseau coloré.

La marge postérieure de l'aile antérieure (dessus) ne porte que peu d'ornement excepté dans le voisinage de la queue de l'aile. Il en est de même de cette prédominance des traits transversaux des ailes postérieures.

La plupart des taches marginales nervurales sont petites.

\section{Xyleutes (Endoxyla) strix L. O"}

$$
\text { (l'. II, fig. 12). }
$$

Collection de Staudinger.

L'exemplaire mâle est plus petit que les femelles examinées. et a une couleur plus uniforme à cause du manque presque total des parties blanches.

Le dessus de l'aile antérieure.

Le réseau coloré diffère de celui des femelles, parce que les 
collules contiennent ici le plus somvent deux rangées de mailles, ce qui donne une certaine régularité au dessin. Dans beaucoup de mailles on rencontre une figure centrale (un point ou une tache).

La marge postérieure a moins d'ornement.

De toutes les taches celles à la fin du champ discoïdal ressortont le plus; les taches marginales nervurales sont bien déveluppées.

Le dessus de l'aile postérieure.

On observe le long du bord externe un lacis coloré qui a uno grande similitude avec celui de l'aile de devant.

La marge antérieure ne porte aucun dessin.

\section{Le dessous de l'aile antérieure.}

La marge antérieure est dessinée distinctement; on trouve sur cette surface le mème dessin quau dessus, mais un peu plus foncé.

Le dessous de l'aile postérieure.

La marge antérieure est semblable à celle de l'aile de devant.

Dans un des exemplaires les ailes n'étaient pas étalées; le Lépidoptère était monté dans la position de repos.

Les ailes sont rabattues et le Lépidoptère, vu de dessus, ne laisse roir que les ailes antérieures. Vu de dessous, on observe la face inférieure des ailes postérieures et d'une partie des ailes antérieures. Or on peut remarquer que ce sont les marges antérieure et externe qui ne sont pas couvertes et que ces marges portent un dessin plus vif que les parties cachées. Dans ces dernières parties les dessins avaient disparu tout à fait ou étaient plus ou moins affaiblis.

La marge externe reste découverte à cause de la petitesse de l'aile postérieure; la marge antérieure au contraire, parce que laile de devant forme une niche dans le voisinage de la sous-constale et du radius. La membrane ailaire prend ici une autre direction; la surface entre les deux nervures susdites est placée perpendiculairement sur le plan propre de l'aile et forme done un bord 
montant. L'aile postérieure s'arrête contre ce bord quand elle glisse sous l'aile de devant, prenant la position de repos.

Oudemays (54) a étudié ces questions. Nous y reviendrons encore plus tard.

En outre nous tâcherons de rechercher comment la niche de l'aile de devant s'est formée.

\section{Endoxyla ligneus Butl. \\ (Pl. III, fig. 5).}

Collection du Jardin Zoologique d' Amsterdam.

Le dessus de l'aile antérieure.

Dans cette forme le dessus a un dessin tout différent, car celuici consiste en grande partie en stries longitudinales. En examinant plus profondément cette ornementation nous trouvons une connexion entre ces nouveaux motifs et le motif réticulé, dont des restes importants se sont encore maintenus.

On rencontre ces stries le plus souvent au milieu des cellules, suivant le cours des nervures; cependant les deux dernières, paralliles au bord postérieur, ne sinquiètent pas, pour ainsi dire, des coudes de la nervure anale, mais se prolongent dans la míme direction depuis le début jusqu'au terme. On remarque dans les cellules distales des figures longitudinales, construites évidemment de traits et de parties de réseau. Dans le voisinage de la queue de l'aile il y une figure grise dans laquelle on pent distinguer les traits transversaux. Intérieurement à cette figure il y a une coloration noire en forme d'ombre.

\section{Le dessus de l'aile postérieure.}

Celui-ci est plus simple; on voit encore dans la marge externe unr tache triangulaire avec un peu de réseau pigmenté. Lesstries longitudinales ressortent moins fortement.

Le dessous' de l'aile antérienre.

Plus simple et plus régulier que le dessus à cause du manque de la figure grise; les traits se sont ici maintenus.

La marge antérieure a un dessin plus développé qu'au dessus. 
Le dessous de l'aile posterieure.

Le remplissage foncé longitudinal des cellules que nous arons observé au dessus et qui porte les traces de son origine de traits joints réticulairement, est encore plus distinct au dessous et manifeste une grande similitude aree les deux firetes des ailes de derant.

\section{Langsetorfic frenkii $\mathrm{Hb}$.}

$$
\text { (Pl. III, fig. 6). }
$$

Brésil. Collection du Jardin Zoologique d'Amsterdam.

Cosside brune-grise de dimensions moyennes.

\section{Le dessus de l'aile antérienre.}

La marge antérieure possède des figures noires, bordées des deux côtés par une bordure étroite de couleur orange; les figures sont de différentes dimensions; d'abord nous rencontrons un certain nombre de taches plus grandes, puis quelques-unes plus itroites et plus petites et ensuite une grande tache et quelques points pigmentés beaucoup plus petits.

Une bande foncée se prolonge parallèlement au bord externe; dans la partic la plus antérieure la bande prend une direction un peu différente du reste. Elle atteint ici les bords antérieur et externe. Un petit segment de la pointe de l'aile n'est pas couvert par la bande. Dans ce segment il y a une tache foncée qui, comme le segment, est bordée d'une ligne jaune. Une branche assez rague prend son origine dans le côté intérieur de cette bande et se prolonge rers la racine, pour se courber au milieu de l'aile et s'unir avec la dernière partie de la bande transversale; c'est ainsi qu'il se forme un champ triangulaire, où nous rencontrons encore quelques traits transversaux. I,e long du bord externe, l'aile a un colneis fonce; les taches malginales nerrurales sont présentes, mais peu développées; autour de ces taches, ça et là, une bordure jaune. Dans le ruban, entre le bord externe et la bande transversale, on remarque quelques barres transversales. I la limite de ce champ et de la bande, chaque nervure porte un point jaune, petit mais net. Derriere chacun de ces points la bande se gonfle 
en dehors. Le dernier point (le 6 ème) est situé plus proximalement cà.d. dans la bande; ce point est allongé dans la direction de la nervure.

Ces barres comme celles du champ triangulaire sont le plus visibles dans une lumière tempérée, c'est à dire, quand avec la main on empêche la lumière d'arriver directement.

Il y a quelques taches remarquables dans la partie basilaire de l'aile: Une, argentée, bordée de noir, devant la nervure anale, et une autre, derrière cette nervure, argentée aussi, courbée en dedans en forme de croissant. Cette tache touche le bord postérieur, longe ce bord, et est dissoute en avant en un certain nombre de taches plus petites dont quelques-unes sont également argentées, les autres jaunes.

Elles forment un cercle qui enferme une partie plus foncée.

La marge postérieure a quelques traits indistincts.

Le dessus de l'aile postérieure.

Celui-ci a une conleur brun uniforme. Sur les nervures longitudinales on voit quelques petites stries jaunes, qui sont en rapport aver les points mentionnés plus haut dans l'aile antérieure et le long du bord externe, les vestiges de taches marginales bordées de jaune.

\section{Le dessous de l'aile antérieure.}

La marge antérieure est dessinée de la mème maniere qu'au dessus.

La même bande, quoique plus claire, a ici une bordure jaune étroite.

La bordure du coité extérieur est moins développée que de l'autre côté, et entre le plus en évidence là où elle croise les nervures longitudinales, parce que c'est là que les points jaunes sont situés comme au dessus. Le côté extérieur de la bande forme une courbe concave en dedans de point en point. Du côté intérieur la bande a une bordure moins sinueuse, excepté entre $R_{5}$ et $M_{1}$ et entre $M_{1}$ et $M_{2}$, ou deux taches ovales et grises, bordées de jaune, $\mathrm{y}$ pé nètrent.

Le segment clair de la pointe est développé de la même manière qu’à la face inverse. Le champ triangulaire est présent, 
mais contient des éléments transversaux de dessin en quantité plus grande et ressortant plus sur le fond. Je n'ai pu examiner la marge antérieure, parce qu'elle était cachée tout à fait sous l'aile postérieure.

Le dessous de l'aile postérieure.

La bande de l'aile de devant se prolonge sur l'aile de derrière, jusqu'à environ la dernière branche du cubitus et est bordée de jaune, couleur que les fragments des nervures longitudinales qui sont situés dans cette bande, montrent ígalement. Lit, où la bande atteint le bord antérieur, on observe un certain nombre de traits transversaux jaunes entre la sous-costale et le radius.

La marge antérieure possède de longs traits transversaux, dont quelques-uns sont bordés de jaune. Ia partie distale de la cellule, formée par la costale et la sous-costale, est en couleur foncée. Cette roloration se prolonge dans la cellule voisine. Plus intérieurement on rencontre une tache jaune dont le bord postérieur est dentelé; plus loin, dans la même direction, une tache foncée jusqu'à la nervure transversale entre le radius et la sous-costale. Dans la région médiane j'ai compté un nombre assez grand de traits qui, à vrai dire, sont ragues, mais qu'on peut bien distinguer dans une lumière tempérée. Ils sont placés à des distances régulières et sont assez droits.

Les taches marginales nervurales sont d'un coloris un peu plus foncé que l'aile. Elles sont arcompagnées d'une petite tache jaune'; celle de la nervure anale est jaune en grande partie.

Il est intéressant de constater qu'ì l'endroit où le pli anal atteint le bord, une semblable tache se produit.

20. Prionoxystus robinice Boisd.

(Pl. III, fig. 7 et 8).

Kansas City, Missouri. Collection du Jardin Zool. d'Amsterdam.

Exemplaires mîles.

Le dessus de l'aile antérieure.

La marge antérieure porte des traits; ceux de la cellule cos- 
tale ne s'accordent pas avec ceux de la cellule sous-costale. Entre $R_{1}$ et $R_{2}$, comme dans les cellules $R_{2}-R_{3}$ et $R_{3}-R_{4}$, ces éléments ne sont pas très grands, mais bien développés. Une grande partie de l'aile est occupée par. le motif réticulé.

Les mailles sont de diverses dimensions; quelques-unes sont situées sur deux cellules voisines. Dans beaucoup de mailles on observe un centre coloré qui a quelquefois une forme stellaire et qui sans doute doit être considéré comme une partie transformée du réseau pigmenté.

Cette transformation provient, à notre avis, de la disparition d'un certain nombre de lignes de jonction, ce qui cause l'isolement de la figure. Le dessin se développe ça et là en figures qui, sous certains rapports, ont l'air de bandes.

Nous reneontrons dans les cellules $\mathbf{R}_{4}-\mathbf{R}_{5}, \mathbf{R}_{5}-\mathbf{I I}_{4}, \mathbf{M}_{4}-\mathbf{I I}_{2}$ et $\mathrm{MI}_{2}-\mathrm{M}_{3}$ des poutrelles longitudinales dentelées, qui paraissent être formées par le noircissement du réseau à la périphérie, tandis qu'au milieu de la cellule le dessin est affaibli.

Ces poutrelles sont groupées en une bande transversale complète. Cette bande se tourne en avant dès le cubitus en formant un angle d'environ $60^{\circ}$.

Une autre figure en forme de bande avec une branche courte en dehors occupe le milieu de l'aile.

Quelques parties du réseau peuvent encore être distinguées distinctement dans cette bande.

Le dessin le long du bord postérieur est un peu embrouillé, parce qu'ici les écailles sont dressées.

Ies taches marginales nervurales sont hien dévoloppées; évidemment elles sont en connexion avee le réseau. Dans un autre exemplaire de la même espèce le réseau a une forme particulière. La cellule entre $\mathrm{M}_{3}$ et $\mathrm{Cu}_{1}$, par exemple, est noircie en grande partie. Cette partie noire entoure un certain nombre de grandes taches rondes avec des figures centrales ramifiées en forme d'astre (fig. 10). Dans cet exemplaire la marge postérieure possède un beau dessin réticulé. 
Le dessus de l'aile postérieure.

Cette surface est grise-brune et assez terne avec un dessin vague, mais complet. Dans la région antérieure on observe des traits, dans l'autre partie le dessin réticulé.

Le dessous de l'aile antérieure.

La marge antérieure est dessiné distinctement d'éléments typiques; le dessin ressemble, jusque dans les plus petits détails à

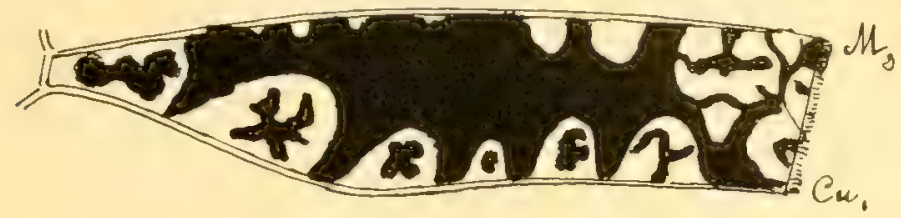

Fig. 10. Cellule $\mathrm{MI}_{3}-\mathrm{Cu}_{1}$ de l'ailo antérieare (dessus) de Prionoxystus robiniae Boisd.

celui du dessus, au moins quant à la forme et à la situation des éléments; en ce qui concerne la couleur, la marge est plus terne.

Les taches marginales nervurales sont distinctes.

La partie basilaire de l'aile est couverte en partie d'écailles piliformes.

\section{Le dessous de l'aile postéricure.}

La marge antérieure est ornée nettement.

Nous observons entre la costale et la sous-costale des transitions entre le motif réticulé et celui des traits. Le dessin est plus developpé qu'au dessus; il y a des taches dans la région médiane.

La partie en éventail est sans dessin.

\section{Hipopta thrips IIb.}

\section{Collection de Katumanbicir.}

Le dessus de l'aile anterienue.

La surface ailaire est d'un ocre jaune pâle; dans la marge antérieure il y a quelques traits bruns plus foncés qui sont placés à des distances régulières. Le dessin des autres régions consiste en traits droits et assez réguliers. 
L'ornementation de l'aile ne saute guère aux yeux à cause du peu de différence des teintes $d u$ dessin et du fond. Les traits restent ponctuellement dans le cours des nervures; quelques-uns seulement forment des bandes et paraissent dépasser les nervures.

Entre deux traits bien développés nous voyons souvent un autre plus vague, de sorte qu'une figure se produit, qui fait penser à une tache claire avec un centre plus foncé.

Dans la partie médiane de l'aile il y a une tache irrégulière, assez grande, un peu plus foncée, qui est entourée d'une bordure blanche. Cette tache s'étend de $\mathrm{M}_{2} \grave{a ̀ ~} \mathrm{An}_{2}$.

Le long du bord postérieur se trouvent des traits transversaux. Le dessus de l'aile postérieure.

Coloré plus clair que l'aile antérieure, mais orné aussi de traits. Le dessous de l'aile antérieure.

La région proximale de la surface ailaire est à peu près dépourvue de dessin, sauf le long des bords antérieur et externe, où il est bien distinct.

Le dessous de l'aile postérieure.

Coloré comme le dessus; la marge antérieure n'a qu'un vestige de dessin.

\section{Holcocerus arenicola Stgr.}

\section{Collection de Kallenbach.}

Ce Lépidoptère ressemble beaucoup à Cossus cossus, mais donne une autre impression par le gris qui domine.

\section{Le dessus de l'aile antérieure.}

Le motif principal est celui des traits, qui sont assez droits au milieu, mais qui présentent toutes sortes de transformations dans les autres parties; ils se ramifient, forment des jonctions, s'affaiblissent on se transforment en réseau. Des bandes transversales noires se prolongent sur l'aile; bandes qui ont une grande similitude aree celles de Cossus cossus; elles ne sont pas non plus complètes, souvent entrecoupées, pour ainsi dire, parce que les 
deux bouts des denx fragments de la même bande ne sont pas réu nis.

Les dessins de l'aile gauche et de l'aile droite sont souvent très différentes en détails. Lal marge antérieure a des traits vagues; la marge postérieure est à peu près sans dessin, excepté dans le voisinage de la queue de l'aile.

Le dessus de l'aile postérieure.

Celui-ci est coloré en clair avec un dessin qui ne ressort guère sur le fond et qui est composé de traits, qui se ramifient, s'anastomosent etc.

Le dessons de l'aile antérieure.

La marge antérieure est ornée de traits. Le dessin est moins net qu'au dessus et n'a pas de bandes.

Le dessous de l'aile postérieure.

Celui-ci a un dessin vague; seulement le long du bord antérieur on observe des restiges d'un dessin. 。

\section{Phragmatoecia castanea Hb.}

Collection de Kallenbach.

Cette forme, avec l'abdomen très allongé, montre peu d'ornementation: pourtant il y a quelques taches sur la face inférieure, comme sur le dessus, ou relativement, elles sont plus distinctes.

\section{Stygia ledereri Stgr'.}

Collection de Kallenbach.

Petit Lépidoptère; les ailes légèrement translucides et l'abdomen allongé.

Le dessus de l'aile antérieure.

Celui-ci est orange et brun foncé. La marge antérieure possède quelques traits; le dessin est composé de quelques bandes et de taches brun-foncé. sur un fond d'orange; les motifs ne sont pas limités très distinctement; pourtant on voit une alternation assez régulière de bandes claires et foncées. On observe le long du 
bord externe une série de taches nerrurales foncées de forme allongée, dont les parties proximales sont reliées entre elles par des pieces de jonction, formant une bande marginale de taches.

Puis intérieurement une chaine submarginale de taches internervurales foncées et une bande transversale foncée, se composant de deux parties qui sont reliées it peine dans la région médiane de l'aile. D'autres bandes suivent; d'abord une bande claire, rompue au milieu, s'élargissant en avant et en arrière et renfermant une rangée de taches noires, puis une bande large et foncée, un ruban clair et ensuite une série de trois taches foncées.

Le dessus de l'aile postérieure.

Celui-ci est entièrement noir avec une grande tache blanche au centre.

Le dessous de l'aile antérieure.

Le long du bord antérieur: quelques barres foncées; du reste plus vague que le dessus.

Le dessous de l'aile postérieure.

Comme le dessus; dans la marge antérieure des barres claires et foncées qui alternent.

\section{Stygia australis Ltr.}

Collection de Kalifanbach.

Petit Cosside comme l'espèce précédente.

Le dessus de l'aite antérieure.

Blane brunâtre; dans la marge antérieure des traits qu'on observe ausi dans les autres parties de l'aile, quoiqu'ils soient quelque peu indistinets.

Le dessus de l'aile postérieure.

La tache blanche au centre est plus grande que chez Stygia ledereri, de sorte que les marges seules sont colorées; la couleur de ces marges est noire. 
Le dessous de l'aile antérieure.

La marge antérieure est ornée de barres; le dessin est plus vague qu'au dessus.

Le dessous de l'aile postérieure.

La marge antéricure porte des traits.

\section{Ia famille des Arbélides.}

Cette petite famille qui fut établie par IIAMsis (32) comprend un ou deux genres, qu'on comptait autrefois parmi les Cossides, mais qui s'en distinguent par l'absence d'un frein (frenulum) et parce que la nervure qui divise le champ discoidal, n'est pas bifurquée. Les espèces qui vivent aux Indes et à Ceylan sont d'une grandeur moyemne; les antennes des mîles sont bipectinées jusqu'au bout. L'abdomen est beancoup plus long que les ailes postérieures. Les chenilles vivent dans le bois, comme celles des Cossides.

1. Arbela sp.

(Pl. III, fig. 9 et 10 ).

Iles de la Sonde. Collection du Jardin 'Zool. d'Amsterdam.

L'exemplaire qui était à ma disposition, se trouvait dans un état déplorable; les ailes n'étaient pas étalées de sorte que les pustérieures étaient couvertes pour la plus gramde partie. C'omme elles étaient un peu éloignées l'une de l'autre, j'ai pu encore observer quelques détails du dessin caché.

Le dessus de l'aile antérieme.

La couleur de fond est blanche, le dessin brun en diverses teintes. Le long du bord antérieur on observe un certain nombre de taches brun-clair, qui sont le plus développées dans la partie proximale (dans laile gauche la partie distale est aussi bien dessince). Près du sommet les taches sont assez nettes et se maintiennent dans le cours des nervures. Dans la région distale il y a des taches rectangulaires ou en forme de parallélogramme. 
Elles sont souvent encadrées entre deux traits plus foncés; la direction des traits obliques n'ent pas partout la même, tantôt ils se dirigent en avant et en dehors, tantôt en avant et en dedans; plus intérieurement se trouve une large bande qui est entrecoupée par les nervures longitudinales colorées en blanc; la hauteur des composants de cette bande est à peu près le tiers de la longueur, c'est à dire dans la direction perpendiculaire à la bande.

Ces fragments sont tranchés par des traits plus obseurs ce qui prouve qu'ils sont formés de traits pigmentés, entre lesquels la conleur de fond est devenue plus fonc'e que dans l'entourage; ca et là on aperçoit un trait semblable, isolé, séparé du fragment par la couleur de fond non-changée. Tne grande tache brume s'étend des deux côtés de la nervure transversale et une autre dans le champ discoïdal. Dans les autres régions le dessin devient un peu plus diffus, quoiqu'on puisse encore observer quelques taches.

Nous voyons au bout des nervures des taches brunes, situées en grande partie sur la frange.

\section{Le dessus de l'aile postérieure.}

Le dessin ressemble beaucoup à celui des ailes antérieures, excepté qu’il y a ici une région plus claire, le long du bord externe; les traits, très larges, ressortent nettement sur le fond, se courbent quelquefois et peuvent se réunir en forme de figures en $\mathrm{Y}$ oll $\mathrm{WV}$.

Plus en avant les ailes s'éclaircissent, mais les taches sont encore placées à des distances régulières. La marge antérieure est recourbée en haut, de sorte que la coloration du dessous devient visible.

\section{Le dessous de l'aile antérieure.}

La marge antérieure a un dessin plus distinct qu'au dessus.

Du reste les deux faces ont le même dessin; seulement les taches sont ici moins différenciées et l'ornementation disparaît vers la racine. Dans la partie en éventail le dessin ne fait pas entièrement défaut. 
Le dessous de l'aile postérieure.

La marge antérieure a un dessin bien développé; le dessin s'accorde entièrement avec celui du dessous.

\section{E. La famille des Microptérygides.}

1. Eriocramia sparmannella F.

(II. III, fig. 11).

Collection du Musée de Leyde.

Les deux ailes ont à peu près la même forme et sont assez étroites.

Elles sont pourvues d'une frange large; l'aile antérieure ne la possède que le long du bord externe; l'aile de derrière l'a également le long du bord postérieur.

\section{Le dessus de l'aile anterieme.}

La couleur est pourpre et or. Le dessin se compose de traits pourpre courts et larges qui se tiennent rigoureusement dans le cours des nervures.

Ceux de la cellule costale restent indépendants de ceux de la cellule voisine. Quelques traits sont placés obliquement, d'autres perpendiculairement aux nervures. Les plus étroits contiemnent une seule rangée d'écailles, les plus larges en contiennent deux ou trois rangées. Si seulement une écaille du fond y pénètre, la forme d'un semblable élément est totalement changée. En général l'or et le pourpre sont partagés assez régulièrement; dans le voisinage de la bifurcation du cubitus, le fond d'or domine un per.

Le dessus de l'aile postérieure.

Celui-ci est plus brunâtre, mais avec un éclat de pourpre et d'or, un peu moins brillant que sur l'aile de devant. Il y a toutes sortes de transitions entre ces deux couleurs; en outre les écailles d'une même couleur ne se tiennent pas si soigneusement ensemble, de sorte que toute netteté dans le dessin a disparu. On peut reconnaître le vestige d'un trait lì où seulement quelques écailles pourpre se trouvent encore ensemble. 
Le dessous de l'aile antérieme.

Celui-ci a un dessin analogue à celui du dessus, mais plus terne; le caractère du dessin est dû en partie au dessin du dessus qu'on peut observer à travers la lamelle translucide de l'aile, en partie par les écailles de la face inférieure. C'est le long du bord antérieur et dans la région distale de l'aile, que celle-ci est le plus écailleuse.

Le dessous de l'aile postérieure.

Comme le dessus. Le dessin est un peu mieux déreloppé surtout sur la pointe de l'aile.

\section{F. L'ordre des Trichoptires.}

1. Phryganea varia F. .

$$
\text { (Pl. IV, fig. 1). }
$$

Pays-Bas. Collection personnelle.

Le dessus de l'aile antérieure.

La marge antérieure possède des traits bruns, entre lesquels on voit les parties incolores de la membrane ailaire. Ces traits oceupent l'espace entre la costale et la sous-costale; la cellule roisine a une striation qui en est indépendante. Quant ̀̀ l'autre partie de l'aile, le motif principal est celui des traits transversaux internervuraux, tantôt droits, tantôt sinueux ou tortueux, tantôt oblitérés au milieu, de sorte qu'il en reste deux taches, touchant les nervures. Souvent on observe des jonctions avec les traits voisins, formant de cette manière un réseau coloré; les lignes de ce réseau peuvent s'élargir et de cette manière la partic colorée peut quelquefois dominer sur la partie incolore, qui dans ce cas se présente souvent en forme de petites taches claires dispersées sur un fond pigmenté, par exemple, entre $R_{5}$ et $M_{1}$ dans l'aile gauche. Ces taches sont situées surtout des deux côtés des nervures; c’est la région mediante des cellules qui proside une coloration continue. La marge postérieure est ornée de traits.

Le bord externe possède, au bout des nervures, des taches 
qui sont en connexion avec les traits ou le réseau. L'aile n'est pas colorée d'une façon égale dans toute son étendue; quelques parties claires se sont jointes, pour former des bandes assez larges et il en va de même arec les parties foncées.

Une de ces bandes pigmentées s'étend dans le voisinage des nervures transversales et aboutit au bord antérieur à l'endroit où la radiale a une courbure. On distingue dans cette bande une petite tache blanche au point de bifureation de $R_{4}$ et $R_{5}$; et encore une, entre $I_{2}$ et $M_{3}$. Plus loin, vers la racine, une bande claire apparaît, entrecoupée au milieu par la limite extérieure du champ foncé qui se trouve à l'intérieur; ce champ s'éclaircit dans la direction de la racine. Plus vers la base encore, il y a enfin une partie plus claire dont la limite externe très foncée se courbe parallèlement à celle du champ foncé adjacent.

Observons encore la petite tache blanche dans la région médiane de l'aile entre la média et le cubitus.

Non seulement la membrane est pigmentée, mais aussi les poils qui la-couvrent; les parties claires portent des poils clairs, les parties foncées portent des poils foncés.

Le dessus de l'aile postérienre.

Celui-ci est translucide et incolore; a la pointe il $\mathrm{y}$ a des vestiges de dessin, qui montrent de la ressemblance arec quelques parties de l'aile de devant.

\section{Le dessous de l'aile anterienre.}

L'aile étant translucide, le dessin du dessous est le même que cclui du dessus; il cause, pourtant, une autre impression; le dessous est plus terne, ce qui est dû, à notre avis, au duvet plus faible de cette face; parce que ce sont justement les poils qui augmentent la différence entre ce qui est clair et ce qui est sombre. Les poils sont aussi plus courts. Au dessus, des poils blanes alternent quelquefois aree des poils brun foncé, quoique nous trouvions par un examen plus attentif beaucoup de teintes intermédiaires entre ces deux conleurs; au dessous les différences de couleur sont plus petites; le blanc est plus jaunâtre, le brun inoins foncé. 
Il est souvent très difficile de constater la couleur exacte.

Le dessous de l'aile postérieme.

A peu près sans poils; précisément comme le dessus.

2. Phryganea grandis. L.

$$
\text { (Pl. IV, fig. 2). }
$$

Pays-Bas. Collection De Meyere.

Le dessus de l'aile antérieme.

La marge antérieure est ornée de traits irréguliers et nonparalleles entre eux, qui, par ces caracteres, par leur ramification et par leur jonction, donnent une autre impression yüi l'ordinare.

Les traits dans le reste de l'aile ont, en général, la même forme que ceux de la marge antérieure, quoiqu'un petit nombre soient plus droits; dans quelques régions ils forment un réseau comme dans les Cossides.

Nous observons un noircissement du réseau dans certaines cellules apicales; quelques mailles disparaissent, d'autres se maintiennent comme des taches translucides dans de grands traits Iongitudinaux. De semblables figures se trouvent dans les cellules $\mathrm{R}_{5}-\mathrm{I}_{\text {, }}$ et $\mathrm{II}_{1}-\mathrm{II}_{2}$.

Les mêmes figures apparaissent entre les nerrures longitudinales plus intérieurement; leur cohérence avee le réseau est distinctement reconnaissable; aux termes et aux bords latéraux il y a beaucoup de transitions entre le dessin réticulé et celui des traits. Le remplissage des cellules se manifeste à un plus haut degré que dans l'espèce précédente.

On rencontre quelques-unes des figures décrites entre $R_{3}$ et $R_{5}$ comme entre $R_{5}$ et $\boldsymbol{I}_{1}$. Le noircissement dans la première cellule renferme une petite tache ronde près de la nervure transversale. Une semblable figure, très grande, est située entre $\mathbf{M}_{2}$ et $\mathrm{M}_{3}$; celle-ci est une continuation d'une figure longitudinale qui s'étend entre II et Cu dans la direction de la meine. Cet ornement longitudinal dans lequel il y a aussi une petite tache claire 
et ronde, est séparé d'une figure analogue plus distale par une raie qui accompagne les nervures transversales.

Nous obserrons également dans d'autres aréas des noircissements, mais d'un ton plus léger que dans les cellules mentionnées. La marge postérieure possède un réseau coloré; les taches marginales nervurales sont présentes.

Le dessus de l'aile posterieure.

Les ailes de derrière ne possèdent qu'un peu de pigment, non loin de la marge antérieure, mais n'ont pas de dessin distinct.

Les dessous des ailes sont comne dans l'espèce précédente moins poilus que les dessus; la différence en teinte est aussi moins grande.

\section{Limnophilus marmoratus Curt.}

\section{Collection De MeYere.}

La marge antérieure est incolore, sauf la partie distale; nous trourons ici à l'endroit où dans d'autres ordres d'Insectes le ptérostigme s'est formé, une tache foncée, qui renferme des points plus clairs. La cellule entre la radiale et le secteur de la radiale possède des traits transversaux, vaguement développés, et quelque peu irréguliers.

Dans l'autre partie, pour autant qu'elle n'est pas incolore, le pigment est bien visible. Dans ces parties pigmentées on remarque des restes distincts des deur motifs principaux; dans d'autres la production du pigment a été si grande, que seulement de très petites taches de teinte claire sont restées. Les nervures transversales ont une couleur brune, plus foncées que les nervures longitudinales.

Je n'ai pu découvrir de dessin dans les ailes postérieures.

\section{Limmophilus faricomis $\mathbf{1}$.}

\section{Collection DF MFYERE.}

Les ailes antérieures n'ont que des vestiges de dessin. Il est le plus net le long du bord postérieur où l'on peut encore bien reconnaitre les traits. 
En examinant soigneusement les autres parties, celles-ci paraissent posséder, cependant, de vagues indications d'ornementation; surtout dans les r'égions distales; c'est ici qu'on rencontre le motif des traits.

\section{Limnophitus rhombicus $\mathrm{L}$.}

Collection DE MeYere.

Tout ornement manque dans la marge antérieure. Dans la région distale un vague dessin réticulé qui est devenu dans quelques endroits une grande tache unicolore, avec ça et là seulement des points non-pigmentés.

\section{Limnophilus affinis Curt.}

$$
\text { (Pl. IV, fig. 3). }
$$

Pays-Bas. Collection personnelle.

Lornementation de la marge antérieure est absente. L'aile semble n'avoir aucun dessin, mais, par une observation plus précise, de petites figures colorées paraissent être présentes qui rappellent des traits raccourcis, ramifiés et anastomosant entre eux; les nervures longitudinales sont tacheteres alternativement claires et foncées; les taches foncées forment la continuation des petites figures entre les nervures.

Dans la figure 3 (planche IV) j'ai exagéré un peu le coloris.

7. Neuronia imperialis Snell. v. Voll.

$$
\text { (1'l. IV, fig. 4). }
$$

Collection du IIusée de Leyde.

Les Neuronias, comparées aux autres Trichoptères, sont colorées d'une manière brillante.

\section{Livile antérieme.}

La couleur de fond est d'un brun-jaune vif, celle du dessin est brune.

La marge antérieure rappelle virement celle des Cossides et possède des traits d'une largeur différente; tantôt ils sont très 
visibles, tantôt ils se présentent comme des points. La plupart sont placés perpendiculairement au bord costal, quelques-uns forment un autre angle, et d'autres encore se sont unis en formant me tache qui cependant trahit nettement son origine multiple. Ils ne dépassent pas la sous-costale et sont placés en partie dans le prolongement des figures analogues entro la sous-costale et la radiale.

L'autre partie de l'aile est occupée à peu près entièrement par le motif des traits.

Dans la région antérieure de l'aile ils sont assez droits, au milieu de l'aile ils s'unissent souvent par un de leurs bouts; de sorte que des figures en forme de $\mathrm{V}$ ou W prennent naissance; dans la partie distale de l'aile la pigmentation s'étend, colorant une partic des cellules apicales d'un brun uniforme avec ça et là une petite tache clairc. Cette fusion des éléments du dessin est accompagnée par un affaiblissement de teinte, en sorte que presque toute la surface ailaire distale a une nuance plus terne que la partic basilaire, oủ les traits, souvent très larges, sont indépendants et séparés par des intervalles jaunes.

La marge postérieure est ornée de figures en forme de $\mathrm{Y}, \mathrm{V}$ ou $\mathrm{W}$.

Les taches marginales sont indiquées vaguement; on les remarque seulement par les intervalles claires qui les séparent.

Liaile posténieure.

Celle-ci est d'un brun unicolore avec un luisant violacé. Nous rencontrons une bande, partant du bord antéricur, laquelle s'étend jusqu'au cubitus et dont le bord extérieur est dentelé. Le long du bord antérieur cette bande s'élargit un pers; on remarque ici quelques traits assez distincts.

8. Neuronia imperiatis, var, regina Lachl.

Yokohama. Coll. du Musée de Leyde.

Cette forme est un peu plus grande que la précédente et est plus nettement dessinéc encore et plus foncée.

Livile antériewre.

I a cellule costale est ornée de larges traits rectangulaires brun 
foncé, entre lesquels on aperçoit les larges intervalles brunjaune de la couleur du fond. Sur ce fond il y a encore quelques restes de traits, en forme de tache, plus ou moins allongées.

Les figures de la cellule costale dépassent la sous-costale, en partie même la radiale, pour se terminer au secteur de la radiale. Pourtant on peut reconnaître facilement que ce ne sont pas des raies d'un seul tenant ne s'inquiétant pas des nervures longitudinales, car, quoique les fragments internervuraux de ces raies colorées soient situées dans le prolongement l'un de l'autre, il est néanmoins évident, qu'ils ne sont autre chose que des traits transversaux, qui ne se distinguent en rien des traits ordinaires. Les composants d'une raie ne sont pas toujours de la même largeur; ils n'ont pas toujours la même direction, et quelquefois ils ont, pour ainsi dire, glissé un peu à côté.

En outre les traits ne forment pas partout des rangées continues. Dans la troisième cellule il y en a qui ne se prolongent pas dans la deuxième. Dans le prolongement d'une des raies, qui parcourt les cellules costale et sous-costale nous voyons quatre traits moins larges et une tache dans l'aile ganche se terminant par trois traits.

Dans le reste de la surface de l'aile le motif réticulé domine.

Quoiqu'il y ait un certain nombre de traits, - mais le plus souvent transformés et ramifiés, joints l'un ì l'autre, élargis dans leur partie moyenne, ou devenus plus étroits et affaiblis - le motif réticulé occupe une partie très considérable de la surface de l'aile, indiquant encore sa connexion avec les traits transformés.

Ici comme dans les Cossides les mailles different en grandeur et en clarté; elles se sont développées très bien le long du bord postérieur. Ida nervure transversale entre le cubitus et la nervure anale est entourée d'une large bande colorée.

Les taches marginales nervurales sont grandes et nettes; apparemment elles font partie du réseau coloré; on rencontre dans quelques-unes de petits espaces moins pigmentés.

Au milieu de l'aile trois taches se présentent, formant les sommets d'un triangle, dont les côtés se prolongent parallèlement aux bords de l'aile. Ces taches ont l'air poilu; les poils sont plus 
longs et plus rapprochés l'un de l'autre que dans les autres parties de l'aile. Dans la forme typique $\left(n^{0} 5\right)$ les taches poilues sont également présentes. Au dessous de l'aile antérieure on les observe aussi; mais elles sont moins apparentes, ce qui tient sans doute au nombre plus petit de poils.

\section{L'aile postérienre.}

L'aile postérieure est brunâtre avec un éclat violacé. Une large bande jaune se prolonge du bord antérieur, près du sommet de l'aile, jusqu'au bord externe. Dans cette bande oblique quelques traits transversaux se présentent près du bord antérieur de l'aile; dans la partie postérieure nous voyons les vestiges d'un dessin réticulé.

Les taches marginales nervurales sont présentes, mais n'atteignent pas les dimensions de celles de l'aile antérieure.

\section{Veuronia reticulata I.}

(l. IV, fig. 6).

Allemagne. Collection du Musée de Leyde.

Lirile antérieure.

Ce Trichoptère possède un dessin qui se compose presque entièrement de traits dans leur forme la plus simple; aussi bien dans la partie proximale que distale; dans la dernière région ils sont un peu plus transformés que dans la première, car ils s'y unissent quelquefois en forme de $\mathrm{V}$ ou $\mathrm{W}$, et on romarque ça et là une anastomose; quelques-uns se sont un peu élargis au milieu, un autre est un peu plus raccourci et n'atteint pas les nervures.

La cellule costale présente aussi ce motif; des traits analogues se joignent avec quelques-uns des autres cellules, de la même manière que nous l'avons vu chez Neuronia imp. var. regina. Cette jonction manque tout à fait dans d'autres cas. Les deux ailes de mon exemplaire sont, sous ce rapport, différentes; dans l'aile droite la correspondance des éléments des cellules voisines 
est mieux développée que dans l'aile gauche. Les taches marginales nervurales sont également présentes.

\section{L'aile postérieure.}

Les ailes postérieures ont aussi des vestiges de traits transversaux à un plus haut degré que les autres espèces de Nemronia. On rencontre ces restiges dans les régions distales et dans le voisinage du sommet; les taches marginales sont bien développées et les nervures transversales sont accompagnées d'une bordure foncée.

11 est évident que l'aile postérieure possède le même dessin que l'aile de devant, mais très affaibli et en partie effacé.

Chez Phryganea commixta (Musée de Leyde) l'analogie entre l'aile de devant et celle de derrière est encore plus grande; la dernière ayant mieux maintanu son dessin.

\section{CHAPITRE III.}

\section{Les dirers motits.}

En décrivant les Cossides et quelques représentants d'autres ordres d'Insectes, nous avons vu que le dessin, si compliqué qu'il soit apparemment, se compose d'un nombre de motifs relativement petit. Pour rechercher le caractère plus ou moins primitif de ces motifs, il est nécessaire d'en avoir une image exacte; raison, pour laquelle j'ai eru utile d'en faire précéder une description détaillée.

Une seule fois nous citerons des espèces non-décrites, pour servir d'exemple; dans ce cas j'cmploie toujours des exemplaires de la collection de KaLtexbach.

\section{a. Les traits transersaux internervuraux.}

Ce sont de petites lignes qui s'étendent d'une nervure longitudinale ì la voisine, qui se bornent donc à une seule cellule. En géméral leur direction est perpendiculaire à ces nervures; dans quelques cas ils s'y rattachent sous un angle plus ou moins grand. 
(Arbela sp., Pl. III, fig. 9 et 10); leur largeur est très différente; tantôt elles se présentent comme des traits tracés avec une plume fiuc, tantôt elles ont l'air de petits blocs et leur largeur peut atteindre à peu près deux tiers de leur longueur. Rarement nous les voyons se développer régulièrement sur toute la surface ailaire; je trouve ce cas chez Eriocrania sparmamella F. (Pl. III, fig. 11) et chez Calpe capucina Esp. (PI. III, fig. 12), mais seulement dans l'aile antérieure.

Dans le cas ordinaire on les rencontre dans certaines parties de l'aile; le plus souvent dans la marge antérieure du dessus; mais aussi dans celle de l'aile postérieure et en beaucoup de cas aussi au dessous des deux ailes. Chez Prionoxystus robiniae Boisd. (Pl. III, fig. 7 et 8 ) ce motif est restreint au bord antérieur et à quelques cellules voisines, aussi bien dans l'aile de devant que dans celle de derrière; tandis que les antres régions sont ornées d'un autre motif.

Chez Cossus cossus nous le rencontrons dans une partie très considérable et c'est sculement la région distale qui est décorée d'une autre manière.

Les parties en éventail des deux ailes se comportent, quant i leur dessin, très différenment; tandis que sur l'aile antérieure rotte partic porte le motif des traits transversux frópumment dans une forme simple ou peu modifiée, celle de l'aile postérieure est le plus souvent presque entièrement privée de dessin, ou l'on peut seulement en observer quelques rudiments.

Si nous examinons les cellules séparément, les traits paraissent être rangés régulièrement à de petites distances (Eriocrania sparmamella, Calpe capucina); ou une différenciation a eu lieu, de sorte ' lue quelyues-uns ressortent fortement sur le fond, taudis que d'autres ont acquis une teinte si terue, qu'ils sont à peu près invisibles.

De semblables cas se trouvent dans les Cossides, par exemple, dans Cossus cossus. Une certaine régularité peut se produire, quand les traits sont alterualivement clairs et foncés; cette régularité manque quand les ornements de différentes teintes sont placés arbitrairement. 
Les éléments plus larges sont quelquefois développés d'une façon particulière, et peurent être composés de deux traits foncés qui limitent un champ interjacent moins foncé que les traits, mais plus obseur que le fond de l'aile; ce qui peut nous faire croire que nous n'avons pas ici affaire à un, mais à deux traits.

L'espace enfermé se serait assombri, de sorte que sa teinte se serait rapprochie davantage de celle les lignes qui l'enfermont. Il faut ajouter que cela n'est vrai que pour quelques éléments élargis et qu'il n'est pas nécessaire, (cela serait même invraisemblable), que tous les éléments élargis de ce motif aient tiré leur origine de cette façon. Dans beaucoup de cas il n'y a aucune indication d'un champ plus clair au milieu d'un trait large et l'élargissement semble exclusivement dû̀ à une extension plus grande du pigment d'un seul élément.

Quoique la couleur du dessin soit presque toujours plus foncée que celle du fond, lans une seule espece nous rencontrons le cas inverse: des traits clairs sur un fond pigmenté, savoir: dans Calpe capucina, qui en effet paraît avoir un dessin remarquable, car cette coloration particuliere est accomprgnéc d'une disposition également exceptionelle des écailles. Celles des lignes blanches sont rangées en séries transversales, contrairement à celles des champs colorés du fond et on peut observer près des lignes blanches, des rebords montants; la couche des écailles étant, pour ainsi dire coupée ici en direction transversale. VAN BEMMELEN a observé la même disposition chez maintes Hépialides. Vue au microscope la cellule contient un certain nombre de groupes d'écailles; groupes qui sont rangés entre eux comme les écailles d'un reptile.

\section{b. Les traits arqués ou courbés.}

Les traits arqués se règlent, comme les éléments mentionnés sub a, dans le cours des nervures, mais tandis que les derniers sont droits, une seule fois avec quelques "tressaillements", les premiers ont une courbure distincte. Nous arons un très joli exemple en Dalaca assa, Hépialide qui porte justement un des- 
sin aussi caprieieux, parce que les courbures des éléments ue sont pas toujours ingalcment fortes ou dans la meme direction; tantrit elles ont le côté convere vers la racine de l'aile, tantôt vers le bord externe. Parmi les éléments arqués ce Lépidoptère a des traits droits, placés obliquement dans la cellule et formant arec ceux des cellules voisines une ligne oblique, qui dépasse maintes nerrures.

Dans quelques endroits les éléments arqués se sont unis pour former des figures en forme de ( ), d'autres combinaisons ont la forme de $\times$ ou de sablier.

Des traits courbés se trouvent aussi chez Dicranura vimula; dans la partie basale de quelques cellules nous remarquons des traits droits, plus distalement des figures courbées qui se transforment vers le bord externe en lignes en forme de chevron et ensuite près du bord dans une strie longitudinale médiane.

On trouve les éléments courbés chez maintes Bombycides, Noctuides et Géométrides, où ils se rangent souvent en lignes ondulées.

c. Les traits en forme de sablier.

On trouve sur les ailes de certaines Cossides (voir fig. 9), mais surtout, comme VAY BEMrabex l'a montré, sur les ailes de beaucoup de Hépialides et dans ce groupe avee une régularité remarquable, quelques traits en forme de sablier.

Ce sont des figures, s'étendant d'une nervure longitudinale à la voisine, se rétrécissant au milieu et s'élargissant aux extrémités, de sorte qu'elles renferment un espace ovale dans lequel une tache en forme d'anneau se peut présenter. Chez plusieurs, comme par exemple Charagia mirabilis $q$ on voit ces éléments de dessin sur les deux faces de l'aile de devant et de celle de derrière. Quelques autres espèces qui nous fournissent également de bons exemples de ce motif sont: Charagia ramsayi, Charagia cximia o, Hepialus rosatus etc.

d. Les traits effiloche's.

Le dessous des ailes de Frnessa io porte un dessin intéressant, dont nous indiquerons les éléments sous le nom de truits effilochés, 
(rafelstreepjes), motif que je n’ai pas observé dans les Cossides. Dans cette Vanesse les traits effilochés ont une couleur noir mat qui dans une lumière incidente, sous un certain angle, ressort fortement sur le fond noir luisant, tandis que sous un autre angle, la surface ailaire fait l'impression d'être teintée uniformément.

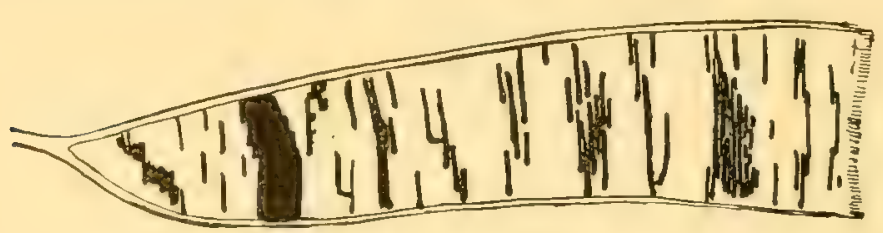

Fig. 11.

Med. 1.

Cellule du dessous de l'aile postérieure de Vanessa io avec les traits efflochés.

Ce sont des trails très étroits, qui vus au microscope, ne se composent que d'une seule rangée d'écailles, qui va dans la même direction que les traits transversaux internervuraux, c'est à dire perpendiculairement aux nerrures longitudinales. Quelyues traits effilochés parcourent toute la cellule; d'autres seulement une partie; ces derniers sont réunis aux nervures par un de leurs bouts, ou en sont totalement détachés. Ce qui est très curieux, c'est qu'ils sont rangés très sourent par petits groupes. Ces groupes forment une transition aux traits transversaux ordinaires. On rencontre encore d'autres formes intermédiaires dans la marge antérieure, où on remarque des traits ordinaires qui sont un peu effilochés ça et là.

On trouve très souvent ce motif dans les Vanessides: sauf dans Vanessa io, déjà mentionné, aussi dans urticae, et principalement au dessous de l'aile postérieure; sur l'aile antérieure nous rencontrons yuelque chose d'analogue, mais plus vague pour deux raisons:

$1^{\circ}$ parce que la longueur et le nombre en est plus petit;

$2^{\circ}$ parce qu'ils sont plus affaiblis en coloris que sur l'aile postérieure.

Puis chez Vanessa L-album Esp. au dessous et dans la marge du dessus; chez I'anessa antiopa L. dans les̀ mêmes endroits; et chez $V$. xanthomelas Esp., $V$. polychloros L., Polygonia C-album L., P. interrogationis F., P. egea Cr., Canace charonia Drury, ete. 
En outre nous tronvons ce motif dans une toute antre branche

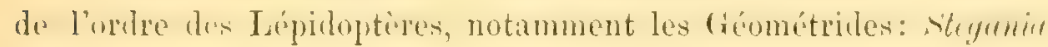
dilectaria IIb., Emhyra pendularia Cl., E. porata I., Selenica bilınaria Esp., Ourapteryx sambucaria L., Angerona mumaria L., A. Mmaria ab. sordata Fusc., Epione apicaria Schiff., des espèces de Synopsia et de Baamia, Hemerophila nycthemeraria IIb., Fidonia rorarie F. ete.

e. Les rangées internerurales longitudinates de taches.

Les éléments sont, comme le nom l'indique déjà, des taches rangées sur les bissectrices des cellules. Elles sont orlinairement ovales, leur grand axe est perpendiculaire aux nervures longitudinales.

Chez Zeuzera pyrina qui nous donne un bon exemple, ces taches se trouvent à des distances régulières; pourtant elles peuvent se toucher et s'unir.

MAYER a trouvé "une loi" en rapport avec la forme.

D'après lui, elles auraient une symétrie bilatérale aussi bien dans la couleur que dans la forme et l'axe de symétrie serait parallele aux nervures longitudinales. Mais dans Zenzera cette loi n'est pas rigoureuse; on pourrait prendre avec le même droit l'autre axe, perpendiculaire à celui de MAYER, comme axe de symétrie. Dans ces deux cas, je présume que le nombre des exceptions serait également grand. Une autre "lui" du meme auteur nous apprend que les taches des cellules voisiues ont des places homologues et qu'elles forment des rangées transversales.

Sans doute, de pareils cas se présentent, mais ce ne sont pas, selon notre avis, des dispositions primaires. Cette , loi" ne se constate pas dans les dessins que j'estime les plus primitifs.

Le plus souvent la tache est une figure ovale ne touchant pas aux nervures longitudinales; pourtant il peut y avoir des différences assez grandes daus la relation des dimensions; quelquesunes sont aplaties et un peu allongées, de sorte qu'elles se rapproclient plus aux nervures et qu'elles ont une certaine ressemblance aree les traits; d'autres sont plus compactes, deviennent isodiamétriques, ou leur largeur surpasse même leur longueur. 
Il. y a aussi des différences de teinte; nous avons vu chez Zensera pyrina que la cause de l'affaiblissement est la diminution en nombre des écailles aussi bien que leur rétrécissement eu largeur.

\section{f. Le motif réticulé.}

Dans beaucoup de Cossides, les ailes sont très sourent ornées, dans la marge externe, d'un dessin réticulé. Dans un seul cas les mailles sont assez régulières; mais ordinairement il n'est pas question de régularité; des mailles de différentes dimensions, grandes et petites, alternent librement; la forme peut être également très différente, quoiqu'on puisse dire qu'elles sont le plus souvent polygonales; un type différent se trouve là où les parties du réseau ne se joignent pas partout; il va sans dire que de cela peut résulter des figures capricieuses.

Les lignes qui forment le réseau, ont une couleur plus foncée que l'espace qu'elles renferment. Quant au diamètre de ces mailles, celui-ci atteint tantôt la moitié de la hauteur d'une cellule, tantôt le tiers, le quart ou même un sixième, dans quelques cas peut-être encore des dimensions plus minces.

Comme dans les traits, nous avons affaire à de grandes différences dans la largeur des lignes du réseau; quand ces lignes s'élargissent, les espaces renfermés diminuent et tout le dessin acquiert un coloris plus foncé. Un pareil assombrissement peut avoir une autre cause; les espaces des mailles peuvent s'assom. brir et se rapprocher, cn teinte, au réseau.

J'ai observé ces deux cas à plusieurs reprises dans les Cossides. Dans l'aile où ce motif apparaît en compagnie de celui mentionné sub $a$, il $y$ a toutes sortes de transitions entre ces deux dessins. Ces transitions sont:

$1^{0}$ des traits arqués irrégulièrement;

$2^{0}$ des traits ramifiés;

$3^{\circ}$ des traits anastomosant l'un avec l'autre;

$4^{0}$ le réseau dans lequel la direction transversale des éléments prédomine. 
S'ai rencontré ordinairement les traits transversaux dans la partie basilaire de l'aile, les transitions au milieu, tandis qu'une marge plus ou moins large était pourvue du motif réticulé.

Dans quelques espèces, Cossus palmaris par exemple, le réseau des parties distales de quelques cullules itait rraisemblablement réduit ou oblitéré, le pigment s'était amassé au milieu, formant ici une poutrelle foncée.

\section{\%. Les lignes et bandes tiansiersales.}

La différence entre ces deux motifs est déjà indiquée par leurs noms. Par bandes nous eutendons les maryues plus larges, quoinue la limite ne soit pas toujours facile ì reconnaitre. Ces motifs sont tellement répandus dans l'ordre des Lépidoptères, qu'il y a peu de groupes qui ne les possèdent.

En particulier nous les remarquons dans les Bombycides, les Noctuides, les Géométrides et les Rhopalocères.

Les lignes et les bandes peuvent être de construction, de forme, de longueur et de direction différentes.

La ligne ou la bande se compose toujours d'autant de fragments qu'elle parcourt de cellules, ce qui est très facile à observer, parce que, ordinairement, soit la direction, soit la largenu chimge un peu à chaque nervure que le motif dépasse. Quelquefois les composants ne se sont rattachés dans leur prolongement qu'incomplictrment, de sorte qüil sumble qu'un ghlissement s'est probluit.

Quelquefois elles sont entrecoupées par les nervures, comme on peut le voir chez Arbela sp. (Pl. III, fig. 9 et 10); une iuterruption distincte se présente souvent là où elles se sont déreloppées du r'éseau coloré, comme dans les différentes formes de Xyleutes (Pl. II). Dans ce cas elles n'ont pas de lignes de délimitation fixement tendues, mais elles ont des bords dentelés, qui s'unissent insensiblement aux autres parties du réseau.

Chez Cossus cossus nous rencontrons des lignes qui ne parcourent qu'une partie de l'aile, nous trouvons, sous ce rapport, toutes sortes de modification: il $y$ en a qui se bornent à deux cellules, mais aussi d'autres qui en parcourent plusieurs. En ce 
qui eoncerne la direction, celle-ci peut également varier, aussi bien dans les différentes espèces, que dans les individus divers de la même espèce.

Quelquefois elles suivent la direction du bord externe, en d'autres cas elles s'en écartent sous un certain angle; l'ouverture de cet angle peut se diriger en arant ou en arrière. J'ai pu observer' dans un exemplaire de rosists cosisus que la grande ligne médiane et la ligne extérieure étaient à peu près parallèles, tandis que dans un autre elles formaient un angle d'environ $60^{\circ}$.

Le nombre des lignes et des bandes n'est pas toujours le même dans les divers individus d'une même espèce; nous en avons des exemples probants dans Cossus cossus. Dans un spécimen de ce Lépidoptère les lignes ne ressortent presque pas sur le réseau et je n'ai pu trouver que de petits fragments ça et là.

Est-ce que nos investigations ont confirmé la théorie d'Enmen quant au nombre des bandes? Nullement.

Aucun fait en fareur du nombre de onze. Que les bandes ne se comportent pas toujours de la même manière dans leurs par. ties différentes, nous pouvons le voir dans les ailes postérieures de diverses Sphingides, où la partie antérieure n'est indiquée que vaguement et la partie postérieure est fortement déreloppée.

C'est dans les Bombycides, Noctuides et Géométrides que les lignes ondulées jouent un role important, chaque fragment d'une telle ligne représente un trait arqué. Si les composants ont la forme d'un chevron, la ligne entière forme un zigzag, comme nous le voyons chez Saturnia pyri Schiff.

Un ensemble gracieux de lignes transversales serrées nous est offert dans le dessin de Brahmaea certhia $\mathbf{F}$.

h. Les taches du bord externe.

Celles-ci peuvent être nervurales et internervurales. Si elles appartiennent au premier groupe, elles sont en relation étroite arec le dessin réticulé, du moins quand celui-ci occupe la marge; elles sont alors fortement développés et ont l'air de faire partie du réseau coloré qui s'est assombri ici. Quand le motif des traits transversaux occupe la partie distale de l'aile, elles se trou- 
vent en connexion aree ce motif. Dans quelques cas ces pigmentations se présentent d'une manière différente de celles qui se trouvent dans les autres parties de l'aile. Ainsi chez Zenzera myrina, les taches qui ne sont pas situées le long des bords présentent toutes les teintes possibles de noir, tandis que les taches marginales maintiennent leur coloris foncé. Il ne faut pas oublicr que le méme phénomène se prorluit aux marges antérieure et postérieure, quoique les taches soient iei des internervurales.

De cette observation on pourrait conclure que la nature différente des taches chez Zenzera ne peut être attribuée à leur disposition diverse: soit sur, soit entre les nervures, mais doit être expliquée par lèr situation en regard̉ de la périphérie.

Nous rencontrons chez Dicranura vimula des taches marginales internervurales, qui sont en relation apparente arec les dessius en forme de chevron de cette espèce.

\section{i. Les figures internervurales longitudinales.}

Celles-ci se présentent comme des lignes étroites dans Dicr $\alpha-$ mura vimula, comme des stries plus étroites ou plus élargies chez Endoxyla ligneus (PI. III, fig. 5) où l'on peut distinguer facilement la relation avec le réseau qui du reste est assez indistincte. Dans Fyleutes (Pl. II, fig. 3, 5, 7 et 8) ce sont des poutrelles dentelées, e'est à dire des figures larges mais courtes. De semblables figures peurent se ranger dans ce genre en forme de bandes transversales.

\section{i. Les colorations des nervures.}

Nous pouvons distinguer: les colorations des nervures longitudinales et celles des nervures transversales. Les dernières sont très communes et peuvent faire partie du dessin ou être sans rapport avec l'ornementation de l'aile.

Les nerrures longitudinales sont colorées dans maintes Piéridres, mais aussi dans quelqunes Cossides, par exemple dans Yylutres d'urrillei et d'une façon élégante dans la Saturnide: Graëllsiu iscubellae Graëlls. 
Bearcoup d'ornements compliqués peuvent prendre naissance de la combinaison de ces motifs simples.

Si l'on songe que les différentes couleur's des ornements peurent augmenter le nombre des combinaisons, ainsi que les modifications de la couleur du foud de la surface ailaire entière ou partielle, qu'en outre le nombre des dessins augmente par' l'affaiblissement ou le noircissement de certains éléments, on comprendra la possibilité d'une quantité énorme de variations quant à l'ornementation.

Ce qui est curieux c'est qu'il se produit toujours un ensemble qui satisfait au sentiment du beau; auquel sans donte la symétrie bilntérale de l'animal et la forme élégante des ailes contribuent.

\section{CHAPITRE IV.}

\section{Le dessin primitif et ses modifications.}

Après avoir étudié les motifs élémentaires, nous pouvons nous demander: lequel de ces motifs peut itre considéré comme le plus ancien. Nous avons déjà vu, que divers auteurs adoptent, comme tel, les bandes transversales d'Eimer.

Mais dans les bandes nous croyons voir un motif composé, car les fragments se présentent aussi séparément, non-joints en bandes qui parcourent toute la largeur de l'aile. En outre il est impossible d'admettre ces fragments séparés comme des parties de bandes décomposées, parce qu'on trouve dans les familles primitiven, comme les Cossides, les Hépialides et les Microptéryides, ces fragments, les traits internervuraux, plus souvent indépendants qu'enchaînés l'un à l'autre, tandis que les bandes, quand elles sont présentes, sont fréquemment dans un état incomplet.

Cela ne plaide pas en fareur de la théorie d'Enmer.

Il n'y a. qu'un pas entre le motif des taches longitudinales, internervurales et celui des traits transversaux.

On aperçoit souvent des transitions entre ceux-ci, ce-qui est aussi le cas aree les traits et le motif réticulé. Qu'ils soient intimément liés, personne ne pourra le nier. 
Ces deux derniers peuvent méme se suppléer dans des cellules analogues de deux individus de la même espèce.

Les rangées longitudinales de taches n'ont pas, à beaucoup près, une très grande extension dans les Cossides, les Hépialides ct les Jicroptérygides; le réseau qui est commun aux Cossides ne se rencontre gulùre dans les autres branches des Lépidoptères, mais les traits se retrouvent partout; par conséquent, quant à l'ancienneté du dessin, l'attention est surtout attirée sur le dernier motif. Il y a encore beaucoup d'autres arguments en faveur de ce dessin et nous allons maintenant les classer aussi bien que possible.

Argument I. Le motif des traits est très commun dans les familles primitives.

On peut considérer comme telles: les Hépialides, les Cossides et les Wicroptérygides.

\section{Hépialides.}

Divers auteurs soit qu'ils aient étudié la nervation, la forme des ailes ou les orgaues intérieurs, sont toujours arrivés à la conclusion que cette famille possède beaucoup de caractères primitifs, à côté de quelques-uns acquis secondairement. Les ailes de devant et de derrière se ressemblent par exemple beaucoup plus que dans les Lépidoptères plus évolués en forme et en constitution de la nervation.

La partie basilaire de la nervure médiane a été conservée, il y a trois paires de ganglions séparées dans le thorax et il existe cinq paires de ganglions distinctes dans l'abdomen; l'intestin antéricur ne posside qu'un renflement, au lieu de jabot; il n'y a qu'une seule ouverture génitale femelle, par le manque d'une poche copulatrice communiquant au dehors par un canal propre; les testicules sout incolorcs et ne s'unissent pas; l'abdomen et le thorax sont très allongés, le dernier portant des ailes souvent très éloignées l'une de l'autre; les ailes antérieures sont pourvues d'un joug, petit appendice de la membrane ailaire près de la racine. 
Dans ma communication provisoire (10) dans laquelle j’ai déja public les principaux résultats de ecte étude, jai mentionné que le motif des traits se trouve aussi dans quelques Hépialides. Plus tard j'ai pu observer qu'on le rencontre chez beaucoup de représentants de cette famille; dans sa forme la plus simple chez diverses espèces de Phassus et quelque peu transformé en traits arqués chez Dalaca assa, par exemple.

\section{Microptérygides.}

Cette famille présente, irréfutablement, une des organisations les plus primitives de tous les Lépidoptères et surtout le genre Micropteryx Hb. (Eriocephala Curt.). WaLter $(76,77)$ a observé ici des mandibules, fonctionnant encore, les lames de la mâchoire n'étant pas encore transformés en trompe, tandis que la lèvre inférieure possède aussi un caractère primitif.

La nymphe est une vraie nymphe (pupa libera) c'est à dire que les ailes, les antennes et les pattes ue sont pas collées au corps, ainsi que dans les autres Lépidoptères. Crapuax (12) a découvert que la nymphe d'Eriocrania avait de grandes mandibules mobiles servant à la chrysalide pour sortir de son cocon; la chenille a 22 pattes, par conséquent elle les possède aus:i aux segments qui en sont dépourvus dans les chenilles des Lépidoptères plus avancés.

Les ailes sont pourvues d'un joug, comme dans les Hépialides. Le nombre des nervures transversales est assez grand; les ailes de devant et de derrière se ressemblent beaucoup; les paires de ganglions du thorax sont encore distinctes et il y a un seul orifice génital femelle.

Les organes reproducteurs mâles, à cause de leurs testicules jneolores, smont moins differenciés que dans les autres Lápidoptires.

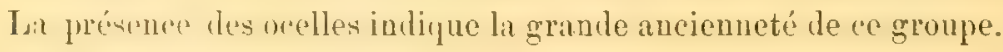

Et maintenant le dessin.

Celui-ci, au moins chez Eriocrania sparmannella F. que j'ai ćtudiée, ne se compose que d'un motif et c'est le motif des traits. 
Cossides.

I.es Cossides présentent, relativement parlant, unc organisation peu éroluée; l'abdomen est allongé, les ailes sont quelquefois it peu près homonomes, ressemblant à celles des Hépialides, les paires de ganglions ne se sont pas encore confondues; l'intestin antérieur n'a qu'un simple rentlement au lieu d'un jabot, ce qui, d'après Petersen, (62) pronve que ees animaux sont restés à un degré inférieur d'évolution.

En aualysant les dessins nous arons trouvé un grand nombre de motifs, comme la striation transversale, des poutrelles et des séries de taches longitudinales, mais, avant tout, le motif réticulé et celui des traits transversaux.

C'est de ce dernier que nous avons $\nabla u$ de brillants exemples comme dans ryleutes sp., Kyleutes strix, Cossus cossus, Cossus palmaris ete.

Il va sans dire qu'il ne suffit pas qu'un groupe donné soit primitif en général pour que tous les caractères de ce groupe le soient également.

Il n'arrivera jamais qu'un tel individu ne possède pas d'autres caractères que des primaires; ordinairement, tout leur habitus et leur morphologie interne est un mélange de primaire et de secondaire et tout groupe primitif présente de nombreuses différenciations sous l'un ou l'autre rapport. C'est aussi le cas avec les Hépialides, famille très simple, mais arec un dessin, quelquefois différencié et spécialisé à un haut degré.

Iais, cependant, on a plus de chances de trouver des caractères primaires chez une forme ancienne que dans des espèces qui sont plus évoluées dans leur organisation générale. Dans un certain organe même, malgré ses différenciations, on rencontrc souvent, si nous recherchons avec soin, des qualités qui rappellent son état primitif, quand cet organe appartient it un animal aucien, géologiquement parlant.

Un exemple probant nous est fourni par beaucoup d'espèces de Ilépialides, qui lessinćes d'une façon brillante et compliquée, portent quelquefois sur le fond de cette riche ormementation, un 
dessin très simple, très originel, le dessin des sabliers, sur lequel vad Bemelen $(7,9)$ a appelé l'attention, comme nous l'arons vu.

Argument II. Les autres motifs peuvent être facilement dérivés des traits transversanx internervwanx.

Nous trourons toutes sortes de transitions entre le motif, à notre sens primitif, et ceux qui sont mentionnés plus haut. Nous pouvons nous imaginer aisément, comment les rangées de taches de Zeuzera ont pris naissance des traits; les formes intermédiaires sautent immédiatement aux yeux, quand on a beaucoup d'exemplaires à sa disposition. Quand le trait s'écarte un peu de la nervure et s'élargit au milieu, il est transformé dans une des taches de Zenzera.

Nous observons ça et là des ramifications des traits; quand les branches s'unissent avec celles d'un élément voisin, le dessin réticulé se produit.

Nous avons déjà énuméré les formes de transition qu'on peut rechercher dans les Cossides entre ces deux motifs, ils peuvent vicarier, comme nous l'avons vu, même dans des cellules analogues. C'est le réseau que nous avons vu se changer en poutrelles ou en stries longitudinales par le noircissement de parties déterminées et l'affaiblissement du dessin de l'entourage (voir fig. de texte 2).

Les traits transversaux internervuraux peuvent se réunir en bandes, dont nous royons la formation, pour ainsi dire, dans les ailes de Cossus cossus; on remarque ici des bandes imparfaites, bandes dont les fragments ont un peu glissé, bandes qui sont doubles quelquefois en quelques endroits.

En résumé nous pourrions dire: Cossus cossus a la tendance à faire apparaitre des bandes dans son dessin, mais n'y réussit pas tonjours.

Les lignes ondulées de beaucoup de Bombyeides ne sont que de modestes modifications des lignes droites.

Les traits peuvent se courber et produisent dans ce cas les figures de Dalaca assa ou de Dicramura vinula. 
Ces traits courbés peuvent se présenter en forme de $<$ et devenir, comme dans Dicranura vimula des stries internervurales longitudinales tendues rigidement.

Les lignes arquées peuvent se réunir pour former des figures en forme de sabliers, comme nous le royons dans quelques Hépialides. Je ne veux pas dire que tous les sabliers de cette famille aient tiré leur origine de cette façon; on peut s'imaginer aussi que les traits ordinaires se sont rétrécis au milieu en s'élargissant aux bouts. On devra rechercher par comparaison exacte des dessins des divorses espeess, quelle origine est la plus probable.

Quand nous nous figurons que les traits ordinaires se fendillent, s'effilochent en traits plus minces, nous avons affaire au dessin du dessous des Vanesses.

Les formes de passage se rencontrent souvent dans la marge antérieure de l'aile de derrière.

Dans cette marge nous reneontrous le même dessin que nous voyons presque partout, mais quelques-unes des figures commencent déjà à s'effilocher.

Cette transformation semble aroir été apte à donner à l'animal un aspect propre à le protéger. C'est pourquoi nous trouvons ce motif justement au dessous des ailes; c'est celui-ci, qui reste visible dans la position de repos, tandis que les dessus brillants sont cachés. Tous ceux qui ont vu subitement disparaître une Vanesse, posée sur un trone d'arbre ou sur le sol, au moment où elle fermait les ailes, étalées d'abord, ne doutera plus de l'aptitude de ce dessin à dérober l'animal à la vue de ses ennemis.

Chacun connaît la livrée des Noctuides, leur ligne ondulée ou "n zigrag, lenr lignes transversales complite et incomplete et lenm taches orbiculaire, réniforme et pyramidale.

Si l'on analyse ce dessin on ne trouve que des traits transversaux. En ce qui concerne les lignes, on le couçoit directement, mais on aura plus de peine a retrouver notre motif dans les taches susdites.

On peut suivre la route par laquelle certains traits ordinaires ont évolué en taches, par la comparaison de différentes espèces, 
d'Ay?otis par exemple. Quand on range ces espèces dans un ordre défini, on voit les traits droits se transformer en traits courbés, et ces derniers s'unir pour former une tache ronde ou réniforme.

Il est évident que la délimitation des taches translucides dans Antheraea yamamai Guér. se compose de traits transversaux, ce qui est encore plus visible dans les taches translucides en forme de fissure dans Caligula japonica Moore.

Ce qui est très intéressant, c'est que ces lignes de démal'cation sont les seuls traits transversaux qu'on rencontre sur la surface ailaire de la magnifique Actias artemis Brehm.

Outre les ornements des nervures transpersales, quelques bandes paralliles au bord cxterne présentent ce motif dans Grällsir. Nous pouvons même dériver l'oeil brillant des ailes postérieures de Smerinthus ocellata de ce simple élément de dessin, mais d'une manière indirecte.

Cet oeil parait notamment aroir érolué de fragments de bandes, comme la comparaison avec des formes apparentées peut nous le montrer. Je voudrais m'arrêter un moment à ce papillon. Les ailes postérieures le la plupart des Sphingides possident peu d'ornementation. Nous observons, pourtant, souvent sur le fond jaune ou rougeatre une bande noire, limite indistinctenent. Cette bande se prolonge à quelque distance parallelement au bord externe.

Là où l'aile présente une échancrure dans le voisinage de la queue, la bande touche le bord externe, par exemple dans Jilinu tiliae. Dans quelques exemplaires de cette espice la partie antérieure de la bande s'affaiblit, tandis que la partie postélieure conserve sa teinte. C'est ce qu'on rencontre aussi dans Smerinthus tartarinovii; celui-là montre dans le voisinage de la yuene une pigmentation noire prononcée lont la continuation est i peine visible. Cette pigmentation est devenue plus compliquée dans smorinthrs lindermami et consiste ici en deux lignes bleues, délimitées en dehors par deux petites lignes noires, tandis que l'espace étroit entre ces deux raies bleues est également coloré en noir. L'ensemble forme une tache qui aboutit subitement à la cubitale 2. 
C'est Smerinthus coeca qui a évolué un peu plus loin; les lignes bleues se sont courbées, clles sont un peu plus courtes que les lignes noires qui les entourent, en sorte qu'une tache en forme d'oeil commence à se former. Cet oeil atteint son évolution la plus lhaute daus Smerinthus ocellata. Les lignes bleues courbées se sont unies en formant un anneau; mais on peut voir distinctement où la réunion a eu lieu, car lì où l'axe, parallèle au bord externe, coupe l'anneau, celui-ci est rétréci; à partir de ces points l'anneau s'élargit graduellement. Nous avons done un anneau bleu, qui renferme un noyau noir et qui est lui-même entouré d'une bande noire. Si alors le rouge de l'aile s'amasse autour de cette figure, c'est l'oeil brillant de notre Smerinthus qui prend naissance.

Il y a encore d'autres taches en forme d'oeil qui peuvent être expliquées aussi simplement, comme nous l'avons fait plus haut quant aux taches translucides.

Ce sont entre autres les taches de Pamassius apollo. Que ces taches soient renfermées souvent entre deux nervures longitudinales, e'est pour Mayer la preuve que l'évolution a commencé dans un point des plis bissectrices des cellules et il a été obligé de chercher une autre explication pour les taches qui occupent deux cellules. Cependant ces deux espèces de taches peuvent être regardées du même point de vue, quand on voit dans les lignes de délimitation des traits transversaux qui se sont courbés en sens divers et qui ont fini par se réunir pour former un anneau. Nous avons affaire à deux traits, si ce sont de petites taches, et ì quatre dans l'autre cas.

Qu'ils n'atteignent pas toujours la nervure, cela ne prouve ricu et n'est pas un argument contre notre point de vue; il y a beaucoup d'exemples de traits qui se sont détachés des nervures.

Argument III. On rencontre le motif des traits dans presque tous les groupes supérieurs des Lépidoptères.

Je n'ai besoin au fond que d'alléguer l'ornementation de la marge antéricure. Celle-ci s'accorde, dans les formes inférieures, 
avec.le dessin des autres parties de l'aile, comme nous l'avons vu dans les Cossides et les Microptérygides, mais daus les especers plus différenciées l'autre partie de l'aile présente un dessin tout différent.

Comme on voit cette ornementation se répéter dans les Rhopalories. lus Noctuides et les Gémétrides toujours dans la même forme qu'elle a chez les types primitifs, on ne saurait refuser a ce motif une grande ancienneté. Nous pouvons faire ressortir cela micux encore en le mettant en rapport arec ce que nous arons dit plus haut.

Il s'agit done d'un dessin qu'on trouve sur toute la face ailaire ou sur une grande partie chez les formes anciennes, dans quelques endroits seulement chez les formes plus évoluées.

Cela ne peut conduire qu'à la conclusion que l'ornementation de la marge antérieure des Vanessides, des Noctuides, des Géométrides et de beaucoup d'autres n'est que le dernier vestige d'un dessin originel perdu.

Si done ce motif se retrouve si souvent, c'est un argument qui plaide en faveur de sa nature primitive.

C'est non moins le cas avec le fait que le même motif surgit partout dans l'ordre des Lépidoptères et occupe ça et là toute la surface de l'aile.

Nous avons déjà mentionné Calpe capucina; ajoutons les Drépanides et les Tortricides.

Dans cette dernicre famille nous ćnumérerons parmi une grande multitude les formes suivantes: Rhacodia emargana, Pandemis ribeana, Teras contaminaria, Tortrix piceana, T. rosana, $T$. sorbiana. Pandemis corylama. Parmi les Drépanides: Oreta pulchripes, Orefu calceoluria, Mrepuna larestinuriu. Je l'ai également retrouré dans quelques Lycénides, savoir: dans Lampides theophrastus.

Dans les Nymphalides on a considéré comme assez primitif le dessin de quelques espèces d'Aryymis; je suis d'accord sur ce point, car, par un examen plus attentif, beaucoup de taches" paraissent être des traits transversaux internervuraux. 
Argument IV. On le rencontre sur quelques ailes de chrysalides et 'puelquefois dans les ailes interpupales, tandis qu'ils ont disparu dans les ailes imaginales.

Poultor a étudié les chrysalides femelles et mâles de quelques espèces de Lépidoptères, dont les insectes parfaits fomelles étaient aptères (voir pag. 5) et a montré que les ailes des chrysalides représentent des stakles ancestraux des ailes de l'adulte et que celles des chrysalides conservent sourent des caractères anciens. Dans l'étude de Poultox il s'agit de l'étendue et de la forme; pout-on trouver aussi des dessins dans les ailes chrysalidaires?

C'est surtout Papitio podalirius, qui a attiré l'attention sous cc rapport, parce que ce papillon ne présente pas seulement une ornementation en relief, mais aussi en couleurs, comme la comtesse Voy Lindes nous en a fait part. Elle et VAN Bemanex l'ont estimé comme un vieux cachet phylogénétique et la première l'a citée pour démontrer la nature primitive des bandes transversales, tentative qui n'a pas réussi, à mon sens, car ce ne sont pas des bandes continues, ce ne sont que des traits qui s'étendent seulement entre deux nervures longitudinales voisines.

En outre VAx Bemmeles a démontré qu'en géméral le dessin de la chrysalide était plus primitif que celui des insectes parfaits. Or, les clirysalides de Gonepteryx rhammi, étudiées par lui, présentent aussi quelquen traits internervaraux comme desin. Tuus arons exposé dans l'historique que le dessin interpupal a été, à plusieurs reprises, un point d'investigation et que c'est surtout Var Bexmelex et la comtesse Vox Lisdex qui ont fait progresser la question. La dernière donne daus son étude française: "Le dessin des ailes", un grand nombre de reproductions d'ailes extraites de leur étui chrysalidaire. Quand on compare ces figures arec le dessin de l'adulte, on aperçoit sur les ailes postérieures de Limenitis sybilla des traits transversaux internervuraux, qu'on ne peut retrouver que difficilement sur l'aile imaginale. Si l'on exanine l'ornementation du dessous de l'aile postérieure de 
Vanessa io dans l'état interpupal, on remarque un grand nombre de traits ordinaires à la place où plus tard les traits efflochés se produiront. - Dans la figure 35 elle reproduit la marge antérieure de l'aile de devant, qui a dans ce stade un nombre d'environ 17 traits; quelque temps plus tard le nombre s'élève à 30 .

Cela indique bien que les traits effilochés tirent leur origine des traits ordinaires.

Argument $V$. Nous rencontrons le même motif dans les Trichopteres.

Comme on le sait, l'ordre des Trichoptères est apparenté à celui des Lépidoptères. C'est un groupe spécialisé quant à la forme larvaire et aux pieces buccales. Or ces Trichoptires ont dans leur dessin les mêmes motifs que les Lépidoptères.

$1^{\circ}$. L'omementation de la marge antérieure. Dans les deux ordres on rencontre les traits, parallèles entre eux, le long du bord antérieur; on n'a qu'à jeter un coup d'œil sur Leuronia reticulata (Pl. IV, fig. 6), Nenronia imperialis (Pl. IV, fig. 4), Nenronia imperialis, var. regina (P]. IV, fig. 5) et Phryganea raria (PI. IV, fig. 1). Si nous examinons la figure de Neuronia imperialis, nous nous apercevons que les traits colorés de la marge antérieure ne dépassent pas la sous-costale; les figures de la cellule roisine ont une autre disposition, qui ne se rapporte en rien à colle de la cellule costale. Chez Nemonia imperialis, var. regina au contraire, les traits de la première cellule sont situés dans le prolongement de leurs analogues des cellules voisines; ees deux cas, nous l'avons vu fréquemment, se présentent aussi dans les Lépidoptères.

\section{$2^{\circ}$ Les motifs du reste de la surface ailaire.}

Je retroure ici beaucoup de motifs des Lépidoptères, et ces motifs se transforment de la même maniere; ils peuvent vicarier, la même relation existe entre le réseau coloré et les traits internervuraux; tandis que Neuronia imperialis var. regina a un réseau magnifipue, qui couvre toute la surface, sauf la partic antérieure, et que ce réseau oceupe la partie distale chez Semoniu imperialis, 
c'est l'espèce reticuluta, par exemple, qui n'a que des traits internervuraux. - Pour être convaincu de la grande ressemblance entre les deux ordres, on n'a besoin que de comparer ce dernier Trichoptère à Liriocrania sparmanella.

Un autre motif, que je rencontre dans Phryganca grandis, e'est celui des colorations longitudinales internervurales, qui comme les stries analogues d'Endoxyla ligneus, ont pris naissance d'un noircissement du motif réticulé.

Les bandes transversales partielles ne manquent pas non plus ici.

Néanmoins cet ordre n'est pas arrivé à un ensemble aussi compliqué et c'est pourquoi, sans doute, il est moins difficile de résoudre pour ce groupe la question du dessin primitif. La réponsc ne peut être autre que pour les Lépidoptères.

C'est aussi chez ces insectes que les traits transversaux internervuraux forment le dessin originel.

La Comtesse Von Linden estime le dessin moins simple que celui des Lépidoptires, opinion que je ne puis partiger; peut-itre explicable par le fait qu'elle n'y retrouve pas si facilement ses bandes transversales.

\section{Argument VI. Le motif des traits se retrouve quelquefois dans les formes aberrantes.}

Dans l'introduction nous avons parlé de l'étude de BRYK sur un individu aberrant de Gonepteryx rhomni, espèce qui dans les cas ordinaires n'a presque point de dessin. Cet exemplaire présente... les traits transversaux, un peu élargis au milieu et rétrécis au bout, traits qui s'étaient done transformés un peu dans la direction des taches internervurales. Ces rangies de taches se rigglent soigneusement à la nervation, ils se rencontrent près du bord antérieur, où ce motif se produit si fréquemment dans une multitude de formes.

Je ne voudrais pas prétendre que toute aberration soit le rappel d'un stade ancestral. Quand dans une personne le phénomène de la brachydactylie ou de la syndactylie se présente, cela ne rappelle certainement pas une époque où la race humaine aurait eu 
les doigts courts et soudés l'un à l'autre. D'un autre côté on connait beaucoup dinomalies qui le font en effet. Dans les ourrages traitant la théorie de la descendance on peut eu trouver assez d'exemples.

Et comme chez le Gonepteryx de BrYK une anomalie se présente, qui ressemble beaucoup à ce que nous estimons primitif, j'ai pensé avoir le droit de faire valoir cet argunent.

Argument VII. Le motif susdit se manifeste plus fortement dans les papillons qui ont été exposés à une température anormale.

Nous avons mentionvé plus haut, que Weismans pourait transformer la forme prorsa par l'application d'une température basse en forme levana, la génération d'hiver, qu'on peut considérer comme plus ancienne que celle d'été. Par comparaison d'uu certain nombre d'exemplaires de ces deux formes, il me semble que dans la forme la plus ancienne, les traits sont marqués plus distinctement, aussi bien au dessus qu'au dessous.

Je crois observer aussi dans les belles figures de Vanessa atalante qui illustrent la communication de MERRIFIELD (51) sur l'influence de la température sur l'ornementation, un dessin plus riche en traits transversaux qu'on ne le rencontre dans les formes normales.

\section{Argument VIII. Le motif des traits est en relation itroite avec la nervation.}

Comme divers auteurs ont démontré que le pigment est conduit par les nervures, il me semble que le dessin primitif, au premier abord, doit être réglé par le cours des nervures et qu'un motif qui ne s'inquiète pas de la nervation ne peut être que dérivé.

Je ne veux pas prétendre que chaque argument ait par luimême une force parfaite de démonstration, mais, somme toute, il existe, à notre aris, assez de raisons pour énoneer cette thèse :

Le systeme des traits transversaux internevcuraux doit être considéré comme le dessin primitif des Lipidopteres. 
Nous communiquerons plus tard, en traitant d'autres ordres apparentés, plusieurs faits pour souligner cette conclusion.

\section{La modification du dessin primitif.}

Si le motif des traits a été le dessin primitif, comment ce motif s'est-il transformé, quelles ont étri les diverses dirertions de l'évolution du dessin de son état primaire à une si grande richesse d'ornements, qui nous remplit toujours de nouveau d'itonnement? Nous avons déjà donné, sous certains rapports, une réponse à cette question, lorsque nous avons démontré comment on peut facilement dériver les divers motifs de l'originel. Les formes de passage que nous retrouvons ì plusieurs reprises, sont ici les balises.

Nous avons rencontré des transitions:

$1^{0}$ entre les traits transversaux et les rangées longitudinales de taches (Zenzere pyrina);

$2^{n}$ entre les traits droits et les traits arqués (Hépialides);

$3^{n}$ entre les traits et le motif réticulé (Kyleutes);

$4^{0}$ entre les traits et les bandes transversales (Cossus cossus);

$5^{0}$ entre les traits ordinaires et les traits effilochés ( $V$ tmessa dans la marge antérieure);

$6^{0}$ entre les traits et les stries longitudinales internervurales (Dicranura vinula);

$7^{0}$ entre les traits et les taches plus grandes (Xyleutes strix).

Toutes ces formes de passage nous indiquent autant de directions qui doivent avoir été suivies.

Chaque motif dérivé peut se différencier de nouveau.

Le résean coloré peut se transformer:

$1^{0}$ en taches (Xyleutes strix);

$2^{n}$ en bandes transversales (Xyleutes d'urvillei);

$3^{0}$ en bandes on poutrelles longitudinales (Cossus palmaris);

4" en stries longitudinales (Endoxyla ligneus).

Les bandes transversales peuvent s'évoluer en taches en forme d'oeil (Smerinthus ocellate). 
Les traits arqués peuvent former:

$1^{0}$ un dessin de sabliers (Dalaca assa);

$2^{0}$ certaines taches (taches translucides de Saturnides et trehes en forme d'anneau de Pamassius);

$3^{0}$ lignes ondulées (Bombycides).

Puis nous avons pu eonstater beaucoup de transitions vers un dessin unicolore.

$1^{0}$ par l'affablissement et le disparition du dessin;

a. les éléments du dessin deviennent plus étroits et disparaissent (Xyleutes strix; e'est ici le dessin réticulé qui disparaît);

b. par l'affaiblissement des couleurs; (on pent l'observer très souvent dans les ailes postérieures;

c. par la diminution du nombre et de la largeur des écailles (Zenzera pyrina).

$2^{0}$ le dessin devient diffus;

les écailles colorées se détachent, pour ainsi dire, du dessin distinct et se dispersent parmi les écailles du fond (Tyleutes PI. II, fig. 7).

$3^{0}$ par l'extension du dessin;

les éléments $d u$ dessin s'élargissent et s'unissent.

40 les espaces renfermés par les eléments du dessin prennent graduellement la couleur du dessin.

5n la couleur du fond et celle du dessin se rapprochent; dans ce cas la surface de l'aile ì l'air unicolore; mais, à un examen exact, elle présente un dessin bien distinct (Cossus sp. Pl. I, fig. 9 et sur les ailes postérieures du mâle de Charagia mirabilis). Quelque chose d'analogue nous est offert par le dessous des ailes de derrière de quelques Vanessides, quoiqu'une différence en luisant puisse se manifester entre le fond et le dessin.

Comme réșumé nous faisons suivre un schéma pour les directions d'évolution.

J'ajouterai que ce schéma n'est pas complet et qu'il ne prétend 


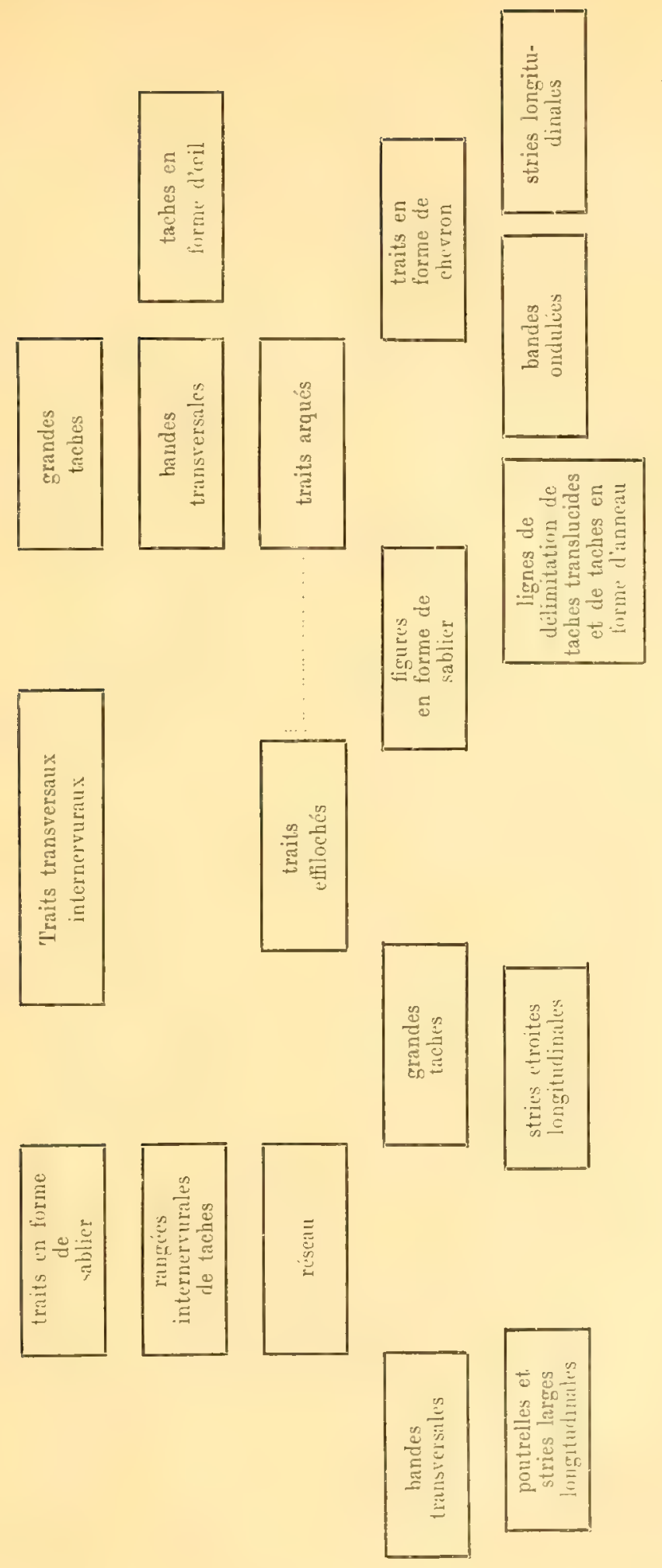


pas l'être d'ailleurs, parce que je n'ai examiné qu'un certain nombre de formes. Les motifs des colorations des nervures ne sont pas représentés; je les traiterai plus tard.

\section{CHAPITRE $V$.}

\section{L'origine du dessin primitif.}

La question de l'origine du dessin doit être étudiée parallèlement ì celle de l'origine des Lépidoptires eux-mèmes. Nous afous done besoin d'abord de rechereher les faits qui sont connus quant à la descendance de cet ordre, afin de pouvoir résoudre la question posée.

Dans les systèmes des différents auteurs nous trourons l'ordre des Trichoptires toujours ì côté de l'ordre des Lépidoptères. Le premier est moins différencié que le dernier par le fait qu'il $y$ a des poils au-lieu d'écailles, encore qu'une partie des poils aient quelque peu l'air d'écailles.

Comme les Trichoptères, sous quelques rapports, sont plus primitifs que les représentants de l'ordre apparenté, un certain nombre d'investigateurs dérivait ces derniers des premiers; ils croient done que les Lépidoptères ont tiré leur origine d'une forme qui derait déja itre considúre comme Trichoptìre, d'antres, au contraire, admettent l'ancêtre commun à une époque plus lointaine et considerent les deux ordres comme des branches indépendantes d'un groupe primitif, d'où ils auraient évolué, séparment, dans des directions differentes, mais partiellement parallèles.

Si la connaissance du dessin suffisait pour tirer une conclusion ì ce sujet, on pourrait être d'accord arec la première conception; il y a, en effet, des 'Trichoptères, ornés d'un dessin qui doit être pris comme le plus primitif dans les Lépidoptires. Nous n'arous qu'à nous rappeler une forme comme Neuronia reticulata.

Mais, nous l'avons $\mathrm{vu}$, il y a beaucoup d'autres faits dont il faut tenir compte. 
Un des systèmes d'organes qui joue un rôle important dans les Insectes, c'est le système des nervures des ailes, parce qu'une ressemblance dims la nervation apporte avec elle une ressemblance dans d'autres directions. En gínéral la nepration des Lépidoptires est assez simple; le nombre des nervures longitudinales est petit; celui des nervures transversales est moindre encore.

Nous en retrourons quelques-unes au bout distal de la cellule discoîdale.

Les dimensions des Lépidoptères n'excercent pas d'influence sur la disposition des nervures, quelynes petites formes exeeptées, où une réduction a eu lieu et qui fourmissent en conséquence beaneoup de difficultés aux Lépiloptérologistes (Wonnworti so)).

Spuler (71) publia une étude comparée des nervures chez les Microlépidoptères, traitant aussi de quelques autres familles de Lépidoptères et de Triehoptères et il donna un schéma de nervation, qui représenterait un stade phylétique des ailes des Lépidoptères, mais qui pourrait servir de même aree de petites transformations, pour les ordres apparentés, comme les Névroptères, les Panorpides et les Trichoptères et qui vaudrait, selon lui, de même pour les ailes de devant que pour les ailes de derrière. Ce schéma est comme suit:

Originairement il y avait dans les Lépidoptères cinq nervures dans la partie principale, la partie limbaire, qu'il indique, en comptant de devant en arrière, par les chiffres romains $I-V$ et qui s'accordent arec ce que nous avons nommé; sous-costale $(=\mathrm{I})$, radiale $(=I I)$, médiane $(=I I I)$, cubitale $(=I V)$ et anale ou pli anal $(=\mathrm{V})$.

Dans la partie en éventail il distingue les nervures $\%$ et $\beta$.

Comme on le roit, Spurer ne compte pas la costale parmi les nervures, ainsi que Comstock l'a fait.

C'est à cause de sa conception que les nervures se seraient constituées aux endroits où l'on rencontrait originellement une trachée, qui manquerait le long du bord antérieur.

La sous-costale émet, près de la racine, une courte branche au

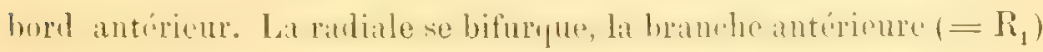


se rend au bord antérieur; d'antres ramifications se produisent de sorte que la radiale acquiert 5 brauches. $\left(=R_{1}, R_{2}, R_{3}, R_{4}\right.$, $\mathbf{R}_{5}$ ). Puis suit la médiane, qui se bifurque aussi; Ia branche antérieure se ramifie encore une fois; il en résulte: $\mathbf{M}_{1}, \mathbf{M}_{2}, \mathbf{M}_{3} . \mathrm{La}$ cubitale ne se ramifie qu'une seule fois en $\mathrm{Cu}_{1}$ et $\mathrm{Cu}_{2}$. La fusion des branches d'une nervure et de diverses nervures cause des complications secondaires.

Dans l'aile de derrière, la radiale a deux branches, excepté dans les ILépialides et les Micropterygides. SPLLER retrouve ce schéma dans les ailes chrysalidaires, encore que les insectes parfaits se soient beaucoup transformés en ce qui concerne la nervation. - C'est le cas dans Dicranura vinula et dans les ailes postérieures de Trtnessl in. Dans cette dernière espèce la sous-costale et la première branche de la radiale paraissent se fondre.

Pienis brassicue semble s'ćcarter de ce schéma, mais les ailes de la chrysalide présentent déjà quelque rapprochement, qui devient encore plus grand dans les ailes de la chenille, encore cachées sous la peau; mais le schéma complet, en ce cas la séparation de la radiale et les trois branches de la médiane, se retrouvait dans une chrysalide anormale de cette espèce.

Il rencontre le mime schima dans les Trichoptires, notamment chez Philopotamus scopulorm et chez Stenophylax concentricus, arec cette différence que daus le premier la médiane possède quatre branches, tandis que le dernier comme beaucoup d'autres sc comporte, au contraire, comme les Lépidoptères.

Une partie antérieure de la cellule discoïdale est séparée par une nervure et forme la cellule accessoire.

Dans les Trichoptères le nombre des nervures transversales est un peu plus grand que dans les Lépidoptères; la plupart de ces nervures se présentent aussi ça et là dans le dernier groupe; cependant la jonction entre $I V_{2}$ et $\mathrm{V}$, commune dans les 'Trichoptères, fait défaut dans les Lépidoptères.

Les ailes postérieures des 'Trichoptères sont construites d'une façon analogue aux ailes antérieures; il en est de même chezles Mieroptérygides et les Hépialides; dans les Lépidoptères supé- 
rieurs les nervures de l'aile postérieure subissent une réduction en nombre des branches.

Se basant sur toutes ces données, on peut done établir une ressemblance curieuse entre la disposition des nervures dans les deux ordres; ils ont même une petite branche de la sous-costale de commun.

Le joug, l'appendice de la membrane ailaire, qu'on peut obserrer dans les Hépialides et la Microptérygides, se retrouve aussi dans les Trichoptères.

Un autre système d'organes que nous derons comparer, c'est celui des pièces buccules. Celles-ci s'écartent dans les Trichoptères assez de la construction générale de ces appendices chez les autres insectes; c'est ainsi que manquent les mandibules; les mâchoires sont très réduites: leurs lames internes sont soudées i la lèvre inférieure, partie buccale qui a aussi une forme modifiée. Dans les Lépidoptères les appendices buccaux sont encore plus transformés. les mandibules sont réduites à de très petites pièces chitineuses, les mâchoires sont transformées et allongées en spiritrompe, la lèvre inféricure est peu développée, sauf les

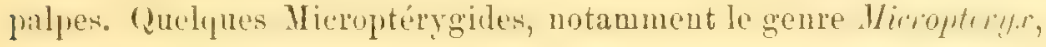
ont, pourtant, encore des pièces buccales du type broyeur, qui ont une construction comme nous la connajssons dans les formes non-spécialisées des Insectes.

Sous ce rapport la ressemblance de ces deux ordres n'est pas si frappante, mais les Jicroptérygides nous prouvent que la spécialisation de la spiritrompe a eu lieu après la formation de l'ordre des Lépidoptères.

Qu'est-ce que nous apprend la comparaison des formes larcires?

Les pièces buccales des larves des Trichoptères ressemblent beaucoup i celles des chenilles; la chenille possède en outre de chaque côté de la tête six stemmates, chacune avec une lentille, les larves des 'Trichoptères n'ont qu'uno ocelle mais qui se compose de six stemmates (Pinkritu, 57).

Ces dernières ont des glandes séricigènes ainsi que les che- 
nilles, mais pas de fausses-pattes, mènent une vie aquatiquo et respirent l'air dissous par des branchies trachéennes.

Encore qu'il y ait des points de ressemblance pour établir une fitroite affinité, il existe aussi des différences. Aussi Haxdursci ne veut-il pas admettre que les Lépidoptères soient dérivés des Trichoṕtères. Il cherche les ancêtres dans un ordre, apparenté avec les deux autres et encore plus primitif sous beaucoup de rapports.

Ce sont les Panorpides.

La disposition des nervures est en plein accord, par exemple pour les espèces de Bittacus, avec le schéma de Spuler.

Une différence avec celle des Lúpidoptires et des Trichoptires c'est qu'on reneontre dans les Panorpides plus de nervures transversiles; un joug est présent, comme dans les familles primitives des Lépidoptères, les ailes sont homonomes; les pièces buccales sont moins spécialisées, encore que quelques parties se soient accolées. La forme larvaire nous fournit un des points les plus frappants de resscmblance arce les Lípidopteres. Les larves mènent une vie terrestre, elles ont trois paires de pattes thoracales et huit paires abdominales, qui sont de fansses-pattes comme dans les chenilles.

D'après Ifaxdmiscul (28) ces deux espèces de chenilles ne différent que dans la fusion des deux segments préanaux.

Il y a donc beaucoup de raisons pour chercher les ancêtres de nos Lépidoptères dans le groupe des Panorpides.

Sans doute les Panorpides vivantes elles-mêmes, se sont aussi transformées sous quelques rapports.

Examinons done les représentants de cet ordre et demandonsnous si cette dérivation s'accorde avec les faits paléontologiques.

Les Lépidoptères les plus anciens ont été trourés dans les terrains du doggere et décrits par Brtuen sous le nom de Palacontina oolitica, tandis que OpPenierm à peu près à la même époque trouva deux formes dans des couches semblables de la Sibérie. Quelques-uns comme Scudder, Brauer et Hasse ne voulaient pas admottre leur nature de Lépidoptères. 
Haxdinsen (28) dit: "Mit Leidenschaft rertraten sie die Ansicht, es könne sich nur um Cicaden handeln, ähnlich jenen Formen, die sich im lithographisehen Sehiefer Bayerns finden und als Cicaden oder Fulgoriden gedeutet werden".

On a cru impossible, que les Insectes suçant le nectar, comme les Lépidoptères d'aujourd'hui, aient pu exister avant le début des plantes à fleurs.

Cet argument tombe cependant, parce qu'on a rencontré dans Wicroptery. un Lépidoptère, qui n'a pas non ylus de pieces buceales du type suceur.

La preuve qu'un combat sur un sujet scientifique n'est pas toujours livré avec des armes loyales, nous a été donnée par l'information intéressante de HAXdIrsce: ScudDer avait intentionnellement donné une figure fausse, pour procurer la victoire à son opinion. - HANDLIRscu a eu aussi l'occasion de constater les vestiges distinets d'une manipulation sur l'original a Londres, ayant pour but de rendre l'objet plus adopté à sm point de vue.

Pour Haxdunsch la nature lépidoptérique se manifeste en vertu des faits suivants:

$1^{\circ}$ dans quelques fossiles les écailles des ailes peurent être observées distinctement.

$2^{\circ}$ Ils rappellent par la tête petite, la forme des ailes et la nervation, les Limacodides océaniques, qui ne sont pas des succurs de fleurs et dont les pièces buccales se sont arrêtées i un stade primitif.

$3^{\circ}$ La ressemblance des fossiles avec les Cicades est très superficielle et la disposition des nervures ne justifie en aucune manière la dérivation des Homoptères.

$4^{\circ}$ La disposition des nervures de fossiles a beaucoup de ressemblance arec la disposition des trachées dans les chrysalides des Lépidoptères vivants.

Ces fossiles-ci, les Palaeontinides, étaient déjà quelque peu différenciés, les ailes hétéronomes en sont témoíns. Les Limacodides ont pris probablement naissance de ce groupe, mais non la plupart des reprenentants des Iropidoptires. La forme originelle 
de lordre entiere aura récu, plus rraiscmblablement dans le Lias, se distinguant encore peu des Panorpiles et des Trichopteres de cette époque, notamment les Orthophlébides et Nécrotauliides.

Maintenant que nous avons rísumé, d'une manière peu détaillée, ce qui est connu de la phylogenèse des Lépidoptères nous nous demandons de nouveau: qu'est-ce que cette connaissance nous apprend de la phylogenèse du dessin; aurait-on pu en observer déjà quelque chose dans les formes originelles, qu'on peut considérer comme le premier pas à la différenciation si riche de l'ornementation des Lépidoptères? A notre avis, nous pouvons donner une réponse arec suffisamment de certitude, à la question: les Lépidoptères étaient-ils dessinés ì leur début? Beaucoup de faits nous ont donné la conviction que le dessin primitif est colui des traits transrersax internervurax, dessin que nous avons retrouvé dans les Trichoptères. Si les Lépidoptères au début de leur évolution avaient les membranes des ailes encore incolores et si l'ornementation arait pris naissance après ce début, il ne serait pas facile d'expliquer la ressemblance avec le dessin des Trichoptires et les transformations en directions semblables. On pourrait l'attribuer à une convergence, mais cette manière de voir n'est pas vraisemblable.

Fu admettant que les formes originelles étaient dújir dessinces, on n'évite pas seulement toutes les difficultés, mais beaucoup de détails deviennent compréhensibles.

Comme on dérive les Lépidoptères ainsi que les Trichoptères, nous l'avons ru, des Panorpides fossiles, il serait utile de rechercher dans les représentants vivants qui ont conservé beaucoup de caractires primitifs, les vestiges d'un dessin qui s'accorde avec l'ornementation primitive des Lépidoptères.

Les Panorpides, en vérité, ont des ailes décorées; on rencontre dans le genre Panorpa des bandes et des taches (voir 79), comme nous les trourons dans les Lépidoptères: - Dans le genre Bittacus uous pouvons aperevoir une colorition tris remarquable. C'est dans quelques représentants de ce genre, que j'ai observé 
dans la collection de Leyde, que je vois les nervures transversales bordées d'obseur (fig. 12).

Il en est de même de beaucoup d'autres espèces de ce genre.

Nous lisons dans la monographie de WESTWOod sur Panorpa et quelques genres apparentés (79) cotte description de Bittacus pilicornis Westw.: „venis transversis versus apicem alarum fusco nonnihil nebulosis" et KLUG remarque it propos d'un Bittacus chilensis: „die Quernerven von einem schwärzlichen Nebel umflossen," tandis qu'il décrit le Bittacus nebulosus entre autres par les mots: "die Quernerren von einem dunkleren Schatten leicht umflossen."

Ce qui est encore plus curieux dans les espèces de ce genre, e'est la réduction des nervures transversales, de sorte qu'on croit

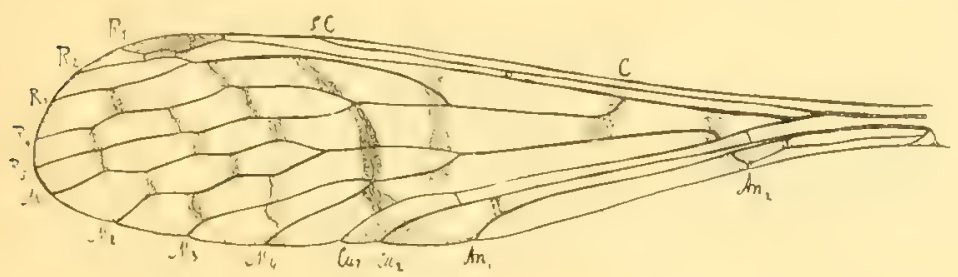

Fig. 12. Aile de Bitlacus corethrarius Ramb.

observer, au premier abord, un trait transversal coloré dépourvu de la nervure qui y appartient.

Ces faits ne donneraient-ils pas uno indiention de la direction d'évolution, qui a conduit au dessin des Lépidoptères?

Cependant, il y a une difficulté. C'est sur la membrane ailaire que se trourent les traits dans les Panorpides, tandis qu'ils sont portés dans les Lépidoptères par les écailles. Ce sont les Trichoptères, qui peuvent nous tirer d'embarras par leurs formes poilues et ornées, comme Phryganea varia. Les pigments, nous les trouvons dans la lame membraneuse, mais aussi dans les poils, dont elle est couverte et de telle manière que les poils des parties obscures sont pigmentés foncés; les poils des parties incolores, au contrnire, n'ont pas acquis de pigment. Nous devons 
admettre que les écailles ne sont que des poils transformés; et il faut done dériver les Lépidoptires d'ancêtres revêtus de poils. Nous pouvons nous figurer par quelle route le dessin coloré a évolué:

$1^{0}$ les nervures transversales avaient une bordure foncée.

$2^{\circ}$ les nervures transversales disparurent, les bordures continuèrent leur existence; les poils qui revêtaient la membrane ailaire, étaient obscurs dans les endroits foncés et clairs dans les parties incolores.

$3^{\circ}$ les poils se changèrent en écailles; par leur largeur augmentée, la lame ailaire devint invisible et perdit sa coloration, mais le vêtement des écailles la conservait en forme de traits transversaux internervuraux.

Il est done tout à fait compréhensible que ce motif doit être considéré comme le dessin primitif. Dìy qu'il n'était plus lié aux nervures par le manque de celles-ci, il s'est comporté plus librement, il put se ramifier, s'effilocher et former d'autres dessins secondaires.

Ce qui confirme cette liypothèse, c'est que les Diptères qui sont dérivés également les mêmes formes que les Lépidoptìres et les Trichopteres, possielent justement, it un haut degré, dans leurs familles les plus primitives, notamment les Tipulides, le motif de la bordure des nervures transversales. Quant à ces Tipulides, je rencontre dans une étude de Nefoncur ') un passage que je trouve trop intéressant pour ne pas le citer ici:

${ }_{n}$ I have drawn and present in figure 12 a typical Tipulid wing in which the principal veins with their full complement of branches are represented in solid black, and the typical cross veins are represented in double contours. This wing is based on a tracing of the wing of Macrochile and differs very little therefrom. Then, in order to see what sort of wing it would be if all the supernumeraries oceurring any where in any crane fly

1) NeEdian, J. G. Report of the Entomologic Field Station conducted at Old Forge, N. $Y$. in the Summer of 1905. Albany, 1908. 
should appear together, I located these supernumeraries, all in their proper places, one by one, and I represent them in dotted lines in this figure. How like a Panorpid wing is the result! If one compares it with the wing of Bittucus, for example, he will see that the differences are very slight, and are confined chiefly to the anal area. There is the same type of branching of all the principal reins, the same upward hitch of rein $\mathrm{Cu}_{1}$ against media, and many of the cross reins oceupy ilentical peritions. Esperially striking are the two cross veins in the first fork of media, one delimiting, the other transversing cell 1 st $\mathrm{M}_{2}$. The suggestion has been made before by others, and I think it very possible, that some Panorpidlike neuropteroid mutant gut its center of grarity hitehed forward, its hind wings reduced, and started the dipterous line of evolution."

Si notre point de vue est juste, le dessin caractéristique de la marge antérieure doit être le rappel d'une nervation abanionnée. Une telle série de nervures transversales, perpendirulaires ou it peu pris perpendiculaires it la costale n'est pas du tout quelque ehose do rare; les exemples sont très courants dans les Nérroptères et dans d'antres ordres vivants, également comme dans le monde des insectes éteints; si l'on feuillette l'Atlas de Handurrscir, les exemples les plus typiques nous frappent toujours de noureau.

Iais on ne les rencontre qu'en nombre restreint dans Bittacus et dans Panorpa, au lieu de la quantité considérable que le grand nombre des traits de la marge antécienere des Lépidopteres ferait supposer. Cependant, il y a un genre des Panorpides, qui s'écarte, sous ce rapport, de Bittacus et de Panorpa; c'est le genre Mirme, dont on peut trouver une figure dims l'1this susdit (Planche $\mathrm{V}$, fig. 18) et dans l'étude de Westwood (79, fig. 2).

Dans la description et la discussion des motifs nous en avons traité quelques-uns un peu défavorablement; ce sont les colorations des nervures; les stries rigides que nous rencontrons sur les nervures longitudinales et sur les nervures transversales qui n'ont pas encore disparu. Je ne pouvais les mettre en rapport 
avec les autres motifs. Nulle part je n'ai aperȩu de formes de passage qui pourraient m'indiquer la relation entre ceux-ci et les autres. Une seule indication, peut-être, nous est fournie par Arbela, qui présente des bandes, produites par le noircissement d'un ensemble de traits transversaux. Ces traits n'atteignent pas ceux de la cellule roisine, de sorte que les nervures longitudinales moutrent la couleur du foud. On observe done dans ce genre des taches noires, entrecoupées par des lignes blanchâtres nervales. Si c'était toute la cellule qui avait subi le noircissement, on aurait les nervures longitudinales dessinées en clair sur un fond obscur.

Nous reneontrons un autre exemple dans Xylentes sp. (P1. II, fig. 5) où les nervures sont en brun faure et les cellules blanchâtres avec un dessin foncé, mais de telle manière qu'il $\mathrm{y}$ a des écailles brunes, mélangées avec les écailles blanchâtres du fond. Si nous nous arrêtons un instant à l'idée que les écailles blanches se sont développées secondairement, qu'ils n'ont pris naissance que dans les cellules, mais pas sur les nervures, nous aurions un cas ressemblant à celui d'Arbela; cependant, l'aile aurait subi dans Tyleutes un blanchissement, tandis que dans Arbela les cellules auraient noirci.

Quand on examine les ordres possédant des ailes incolores, on rencontre fréquemment des formes, dont les nervures sont colol'ées foncées et il en est de même de quelques Lépidoptères qui ont perdu leurs écailles dans certaines parties de l'aile, où donc les membranes translueides deviemnent visibles, antrecoupées par des nervures obscures; les Sésiides et quelques espèces de $M a-$ croglossa nous en fournissent des exemples. Quand les nervures longitudinales peurent être plus riches en pigment que le reste de la membiane, je ne trouve pas étonnant que les ceailles sur les nervures puissent etre dans quelques cas plus foncées que les autres.

Ce serait done un motif, indépendant des traits transversaux. Un eximen attentif des familles dessinces ainsi, serait nécessaire pour dérider laquelle des deux manieres de voir serait la plus juste pour les différentes Papilionides et Nymphalides. Que la médiane 
et ses branches daus le champ discoïdal puissent être colorées également, cela n'offre rien de curieux, quand ces parties de nervures sont encore présentes; ce qui est plus remarquable, c'est qu'on peut rencontrer les mêmes colorations dans des formes où ce champ est privé de nervures, comme dans Hestia et beaucoup de Papilionides. Je ne veux pas tacher de trancher non plus la question de saroir si cette coloration doit être attribuée a la présence de nervules, maintenant oblitérées, dans le temps oủ la coloration prit naissance, ou si elle doit être considerée comme suite de la manifestation de ces nervures pendant le développement ontogénétique.

On trouve encore plus fréquemment une semblable coloration sur les nervures transversales.

Enderlein a démontré que toutes les nervures transversales de ce groupe, génétiquement parlant, ont la signification de nervures longitudinales.

Ne serait-il pas permis alors de voir dans ces nervures les homologues de celles des Panorpides?

1 Cela expliquerait que la coloration de ces nervures est très souvent indépendante des traits internervuraux.

Nous pouvons constater, en tout cas, que ces nervures sont favorisées, en ce qui concerne la pigmentation, aux nervures longitudinales. La présence de ces marques, souvent très capricieuses, ressortant fortement sur le fond, a tellement attiré l'attention des Lépidoptérologistes, qu'ils en ont souvent mentionné la forme dans le nom, qu'ils attribuaient aux porteurs de cette ornementation. On n'a qu'à se rappeler Grapto C-album avec sa marque blane-luisant en forme de $C$, ou $V$ anessu $L$-album, avec la petite ligne brisée argentée sur les nervures transversales. Des figures analogues se rencontrent dans une foule de Noctuides et de Bombyeides.

Il est douteux qu'il faille attribuer les figures nervurales plus compliquées, ainsi que les taches translucides ou en forme d'œil, seulement à une coloration des nervures, car souvent nous voyons des traits y contribuer leur part. 
Une autre question est de savoir si le dessin primitif, comme nous nous le représentons, a été restreint dans les Lépidoptères originels aux ailes antérieures ou s'il s'est étendu également aux postérieures?

Etait-ce seulement le dessus qui était orné ou bien le dessous qui l'était aussi?

Van Bemmelex admet que le dessin ornait les deux surfaces des deux ailes et je suis d'accord avee lui.

Il ne faut pas oublier que les ailes des Lépidoptères primitifs doivent avoir été à peu près homoniomes et plus indépendantes que dans les formes plus hautes, et il ne faut pas non plus oublier que les ailes homonomes des ordres inférieurs ont les mêmes dessins; ce qui est, sans doute, une indication pour la ressemblance des deux surfaces supérieures des formes primitives.

Comme dans beaucoup cie Cossides chaque trait, chaque ornement, si capricieux soit-il, correspond exactement à une figure analogue de l'autre côté, nous sommes contraints d'admettre la même chose pour le dessus et le dessous.

Si done le dessus et le dessous, l'aile de devant et de derrière diffèrent, soit en teinte, soit en motif, un changement secondaire doit s'être produit.

Jusqu'à un certain degré nous pouvons quelquefois découvrir les causes de ces changements.

Nous avons observé dans beaucoup de Cossides, que le dessus des ailes postérieures est teinté plus vaguement que le dessous; le dessin peut avoir disparu sauf quelques rudiments.

En ce qui concerne le dessous de l'aile de devant, e'est ici la marge antérieure, qui conserve sa netteté de dessin et de corleur, souvent ressortant fortement sur le reste monotone.

La partie distale est également mieux développée sous ce rapport que la partie proximale, qui est courerte fréquemment d'écailles en forme de poils.

Dans mes collections il y a un exemplaire de Xyleutes strix et un d'Endoxyla lituratis, dont les ailes n'avaient pas éte étalées, et uni avaient conservé la position, dans laquelle elles devatent ître 
yuand l'animal s'itait place pour se reposer. Ce qui me frappa dans ces exemplaires, c'est que les parties les plus ternes des ailes sont cachées et que les parties qui restent visibles ont un dessin distinct.

En observant l'intensité de la coloration du dessous de l'aile dre devant, on peut en déduire les dimensions de l'ale postérieure.

Tout le dessus de l'aile de derrière est invisible et aussi celui"ii montre-t-il un affaiblissement du dessin.

Cela donne l'explication de la coloration remarquable de la marge antérieure, car le bord de l'aile postérieure n'atteint pas le bord antérieur de l'aile de devant.

Nons avons déjà mentionné qu'il se forme une niche dans le voisinage de la sous-costale et de la radiale de l'aile de devant et également que le bord de l'aile postérieure ne dépasse pas cette niche en glissant sous l'aile antérieure.

C'est à cause de cette disposition que la cellule entre $\mathrm{C}$ et s C reste découverte, ce qui a eu pour conséquence la conservation de dessin dans ce lien.

C'est toute autre chose que le développement postéro-antérieure d'Fner, l'ondulation des qualités d'arrière en avant, de sorte qu'elles se maintiendraient le plus longtemps dans la marge antérieure.

1) plus, nous devons reconnaitre la vérité de ce que OUdemass a dit (56): qu'il ne suffit pas d'étudier les dessins des animaux montés, qu'il faut aussi les observer pendant leur vie; la position de repos domnera souvent la clef dans bien des problèmes de roloration.

liexplication de la formation d'une niche, dont nous avons pralí, se: trouve dans la disposition des appendices de la chrysalide.

Au début de la nymphose, toutes les parties du corps: les ailes, les antennes, les pattes sont encore libres, mais bientôt elles deviennent collées au corps par une matière gluante, pour former ainsi la chrysalide, la "pupa obtecta", que nous rencontrons dans la majorité des Lépidoptères.

Les antennes sont posées sur les ailes antérieures et quand on 
voit, plus tard, les antennes et les ailes dans le même niveau, c'est parce que la partie de l'aile sous l'antenne, donc la cellule costale, a été poussée en bas. En examinant les ailes d'une jeune chrysalide de Cossus cossur, je n'apercus ni la sous-costale, ni la radiale. Ces nervures se montrèrent après avoir enlevé l'antenne superposée. La marge s'est donc enfoncée; de cet enfoncement la cellule costale est le fond, tandis que la cellule souscostale forme le talus.

Nous voyons cette disposition de l'aile fixée dans l'image de ce Lépidoptère pour former ainsi la niche susdite.

Sauf peut-être la coloration des nerrures, on peut dériver tous les motifs du dessin des Lépidoptères des marges colorées qui bordaient les nervures transversales assez nombreuses des ailes de leurs ancêtres, qui auraicnt quelque peu l'air de Panorpides.

Les traits, le motif originel, dont tous les autres peuvent être dérivés, on peut les considérer comme un souvenir des temps oì les Lépidoptères n'étaient pas encore Isépidoptères, comme un héritage des formes ancestrales.

A côté du grand déploiement d'autres caractères dans l'ordre des Lépidoptères, c'est aussi le dessin qui subit toutes sortes de changements.

Quelquefois il a été oblitéré, surtout dans cus parties qui étaient cachées ordinairement, quoique des rudiments trahissent encore l'ornementation d'autrefois.

Jais quand ce dessin ornait les parties visibles, il a pu éroluer pour etre utile dans la lutte pour l'existence de l'animal, pour le protéger contre ses ennemis, produisant un ensemble qui ne ressortait pas sur l'entourage; enfin, le dessin a pu aussi se transformer en vine de buts sexuels.

Handurscir s'est formé une idée d'un Pro-Lépidoptère.

Celui-ci derrait aroir des mandibules imaginales bien développées, des mâchoires et une lère inférieure normales avec des palpes respectirement à cinq et trois articulations, pas de spi- 
ritrompe, pas de jabot pétiolé, les testicules séparées, un seul orifice génital femelle, six tubes de Malpighi, des ocelles, des antennes homonomes, des ailes homonomes écailleuses dans lesquelles les nerrures longitudinales fitaient encore developpées normalement, le thorax assez mobile, pas encore de chrysalide, mais une nymphe („pupa libera”) et la larve polypode, ressemblant à celle des Panorpides.

Nous pourrions ajouter: et un dessin des ailes qui se composa de traits transversaux internervuraux, sur les deux côtés des ailes antérieures aussi bien que des postérieures.

\section{CHAPITRE VI.}

RÉSUMÉ.

Nous pouvons résumer les résultats les plus importants de cette étude, comme suit:

1. Le dessin primitif des Lépidoptères n'est pas celui des bandes transversales d'EnER, ni des taches de Zeuzera, mais des traits transversaux internervuraux, dont les deux motifs indiqués peurent être considérés comme les dérivés.

2. En ce qui concerne le dessin, les Cossides doivent être jugées comme assez primitives nons rencontrons ice frépuemment lo motif primaire des traits et quelques autres qui en sont des modifications légères.

3. Aux caractères primitifs d'Eriocranic on pourrait ajouter l'originalité du dessin; dans E. sparmannella le dessin ne se compose que de traits.

4. Dans les Trichoptères nous rencontrons les mêmes motifs et les mêmes modifications que dans les Lépidoptères. La relation entre motifs primaires et modifiés est la meme dans les deux ordres.

5. Le dessin primitif des Triehoptères doit être le même que celui des Lépidoptères.

6. On peut dériver du motif primitif:

le motif réticulé, les rangées internervurales de taches de Zeuzera, les traits arqués des Hépialides et d'autres familles, 
les figures en forme de sablier, se trourant également et fréquemment dans les Hépialides, les marques en forme de chevron, celles en forme de ganses allongées et les stries internervurales Iongitudinales de Dicramura vimula, les traits effilochés des Vanessides, les lignes de délimitation de différentes taches translucides ou en forme d'œil, les bandes et peut-être les taches sur les nervures.

7. On peut dériver du motif réticulé:

des figures longitudinales, dentelées ou aree des contours assez droits, ainsi que dans Cossus palmaris et Endoxyla ligneus, des bandes transversales et des taches.

8. Les yeux de Smerinthus ocellata sont des dérivés de bandes transversales.

9. Les Lépidoptères primitifs n'étaient pas blancs, mais avaient déjà un dessin emprunté aux types inférieurs.

10. Comme il est rraisemblable que les Panorpides primitives ont été les ancêtres de nos Lépidoptères, c'est l'existence de nervures transversales portant une marge foncée, qui soutient notre opinion quant au dessin primitif d'autant plus, que les nervures transversales ont la tendance à s'oblitérer, tandis que les bordures se maintiennent.

11. La coloration des nervures est peut-être en partie un motif indépendant, en partie le résultat d'une extension ou d'une disparition de l'ornementation des cellules.

12. Les pigments ont été porté primitivement par la membrane ailaire, puis dans les écailles; la membrane les a perdus, la lumière étant interceptée par le duvet des écailles.

Comme je terminais cette étude, je reçus celle du Prof. DE

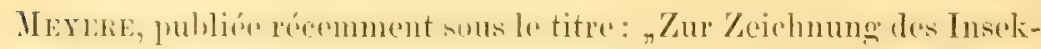
ten - im besonderen des Dipteren- und Lepidopterenflügels." (54)

P'our la vérification de mes conclusions, il n'est pas sans intérêt de les comparer avec les siennes.

Le célèbre diptérologiste commence par les ailes des Diptères. Selon lui, celles-ci sont incolores au début et leur dessin serait 
acquis secondairement. Il tire cette conclusion du fait que la plupart sont incolores. Mais pour la détermination de l'état primitif, le nombre ne peut pas peser dans la balance. Tous les oiseaux virants sont des formes édentées; mais cela ne diminue pas la certitude de leur descendance de formes pourvues de dents.

L'auteur traitant en passant les 'richoptères, énumère les motifs suivants: les bordures foncées des nervures transversales, les pigmentations aux points de bifurcation, et celles au bout des nervures longitudinales, mais non pas les traits transversaux internervuraux, qui, à notre sens, présentent le motif le plus important.

En général le point de départ serait ici un brunissement diffus arec des taches claires nuageuses irrégulières, comme par exemple celles des 'Tripétines. Quoique le dessin eût, selon lui, peu d'importance, il consent qu'on puisse rencontrer recht bestimmte Patronen" et donne comme exemple la bande claire et large des ailes postérieures brun foncé de Neuromia maclachlani. Nous avons vu que les dessins sont plus développés sur les ailes antérieures; ce sont elles qui possèdent l'ornementation caractéristique.

En ce qui concerne les Lépidoptères, les dessins, notamment les colorations des nerrures se régleraient en partie par les éléments morphologiques, qui étaient déjà présents, arant que l'ornementation parût: "es liegt also auch ein ungefïrbtes Vorbild vor"." L'auteur ne nous dit pas oir l'on doit chercher cette forme primaire, encore moins si seulement la coloration des nervures manquait dans les Lépidoptères primitifs, ou si les figures interuervurales faisaient défaut également.

Et c'est là la question dont il s'agit; car les motifs de la coloration des nervures ont peu d'importance, comparés aux ornementations internervurales.

De ce qui suit: ,auch die weit verbreiteten medianen Fleckenreihen halten sich an das Geäder", on pourrait conclure que l'absence antérieure d'une coloration serait applicable égralement it ces motifs, parce que ceux-ci se règlent de la même manièe sur les éléments morphologiques de l'aile. Fint-il chercher cette 
forme primaire après le début de l'ordre des Lépidoptères où dans des groupes inférieurs?

L'auteur n'a pas discuté ce problème, mais mentionne qu'il estime les rangées longitudinales de taches dans les cellules et la coloration des nervures longitudinales comme primaires.

Je ne vois pas elairement pourquoi il faut attribuer aux trois ordres un point de départ aussi différent (je laisse de côté les Névroptères). Et si dans les Diptères, les directions différentes d'évolution auraient tiré leur origine de l'absence totale de pigmentation, comme l'auteur le discute en détail, je ne comprends pas pourquoi les Trichoptères n'auraient pas pu faire évoluer leurs dessins d'un pareil stade; l'absence presque totale de pigment se rencontre ici souvent (par exemple, dans les ailes postérieures). Résumant les opinions de l'auteur, celles-ci le conduiraient done aux conclusions suivantes:

$1^{0}$ la coloration diffuse avec les taches claires serait primitive pour les Trichoptères, mais fort éloignée de l'état primitif dans les Lépidoptères et les Diptères;

$2^{n}$ l'absence de couleur serait primitive pour les Diptères, mais secondaire pour les Trichoptères et les Lépidoptères.

$3^{0}$ les rangées de taches médianes primitives pour les Lépidoptères, et secondaires pour les autres ordres.

Comment faut-il se représenter l'évolution du dessin, lorsque ces trois groupes s'écartaient du groupe commun?

Toutes ces difficultés disparaissent quand on admet comme motif originel pour les trois groupes les traits internervuraux. En ce qui concerne less Lépidoptires, je pense l'a voir sutisamment démontré et par les faits que j'ai rassemblés on peut conclure également que les Trichoptères comme seconde branche, ont apporté avec eux lc même dessin. Je ne parlerai pas des Diptères qui sont hors de mon étude, mais, quand une autorité comme le Prof. DE MEXERE dichare que justement les familles primitives de's Diptères possèdent la coloration des nervures transversales comme ornementation la plus commune, cette déclaration ne peut que confirmer ma conviction, que pour la troisième branche (d'après 
HAxDLnsci) du groupe commun, ce dessin doit être considéré comme le dessin originel.

Je ne reux pas dire que ce soit la le seul motif; il se peut par exemple que la tache au milieu de la cellule qu'on voit dans quelques Panorpides (Panorya punctata Kilug, voir 31) ait été héritée aussi de la forme ancestrale par les Diptères à côté de la coloration des nervures transtersales.

Si l'on admet qu'à leur origine les trois ordres étaient ornés de la même manière, on comprend les directions analogues d'évolution. L'accordance des lignes de descendance déduites de l'observation du dessin, arec celles qui sont obtenues d'une autre façon, domnent un appui solide à notre thèse.

Il y a encore de petites différences dans nos études, par exemple, dans le groupement des motifs.

Le Prof. DE MExene reut les séparer rigoureusement (pag. 88). Iais cette séparation est-elle bien effectuée? L'auteur place entre autres à côté de la coloration des nervures et des rangées longitudinales de taches, l'ornementation de la pointe de l'aile, les bandes, les taches au bout des nervures longitudinales, les bordures des bords de l'aile et le dessin nuageux. Mais l'omementation de la pointe est-elle composée d'autres motifs que celle des autres parties de l'aile? Que les motifs se soient mieux développés dans la pointe, ou que la couleur du fond soit différente dans cette partie, cela ne donne pas le droit de placer la décoration d'une région de l'aile dans la même rangée que les motifs proprement dits.

Irant de quitter l'étude de cet auteur, je tiens à mentionner l'explication qu'il donne de certaines taches en forme d'oeil. II admet qu'un noyau clair se produit dans une tache internervmale foncée. Dans les Cossides j’ai trouvé fréquemment des taches avec des noyaux clairs et ici j'ai pu suivre la manière dont elles s'étaient formées; e'était à cause que deux traits s'étaient courbés ou s'étaient élargis dans le voisinage des nervures; après s'être unis, ils formerent des figures qui renfermaient un espace avec la couleur du fond; la couleur de la partie renfermée a pu se modi- 
fier indépendamment de la couleur de fond hors de cette tache.

Et si les Cossides n'atteignaient pas, sous ce rapport, la hauteur de Parnassius, dont l'espace renfermé est d'une couleur rouge, tandis que le fond de laile est blane, neanmoins on doit expliquer, à notre avis, les taches de cette espèce de la même manière; le cartetire des lignes de délimitation trahit encore son origine d"une manicre distincte.

Le Prof. DE Mexere met ces taches à noyaux clairs en rapport avec les figures en forme de chevron de Dicramma. qu'il concoit comme des anneaux ouverts. Qu'est-ce que c'est que ces anneaux ouverts? Des demi-anneaux? Ne serait-il pas plus simple de les considérer comme des traits plus ou moins arqués?

Je finirai ici la discussion de l'étude de De MeYere, qui n'a pu me convaincre sous aucun rapport, où nos conclusions n'étaient pas les mêmes.

La Comtesse vor Linder a placé au commencement d'un de ses travaux comme devise le mot d'Ermer "que les dessins et les couleurs des Lépidoptères ne sont que des signes qui parlent une langue si claire que quiconque désire connaître la vérité ne peut s'y tromper. Ils nous révèlent, comme les pages d'un livre ouvert, leur origine et leur évolution."

Il se peut que ces mots renferment une part de vrai, il n'en est pas moins certain que ces signes forment jusqu'ici une écriture inystérieuse, dont le déchiffrement est encore dans son début. Que d'autres décident si dans ce qui précède, il a été trouvé une clef, qui puisse nous faire avancer, ne fût ce que d'un pas. 


\section{BIBLIOGRAPHIE.}

1. Bem inelen, J. F. van, Ontwikkeling dev kleuren op de vlindervleugets. Handelingen 2de Nat. en Geneesk. Congres, 1889.

2. Bemmelen, J. F. van, Entwicklung der Farben und Adern auf den schmetlevlings/lugelur.

Tijdschrift der Ned, Dierk. Vereeniging. (2) Deel II, 1889.

3. Bemmelen, J. F. van, Ueber die Phylogenie der Flügelzeichnung bei Tagschmellerlingen.

Zool. Jahrbücher Suppl. XV, Band 3, 1912.

4. Bemmelen, J. F. van, On the phylogentic significance of the wingmarkings of Rhopalocera.

The transactions of the Second Entom. Congress. 1912.

5. Bemmelen, J. F. van, Die phylogenetische Bedeutung der Puppenzeichnung bei den Rhopaloceren und ilıre Beziehung zu derjenigen der Raupen und Imagines.

Verhandl. der Dentsch. Zool. Gesellschaft auf der 23ten Jahresversammlung zu Bremen, 1913.

6. Bemmelen, J. F. va n, I. Onderzoekingen over de ontwilikeling van het kleurenpatroon op de vleugels der Nymphaliden, Pieriden en Papiliooniden, in vergelijking met dat der Hepialiden.

Tijdschrift der NederJ. Dierk. Vereen. (2) Deel XIV, 1914.

7. Benmelen J. F, van, II. Het lileurempatroon van Zelotypia stacyi.

Ibidem.

8. Bemmelen, J.F, va n, Ontwikkeling van het kleurenpatroon op vleugels en lichaam der Lepidopter'a.

Handel, van het 15de Nat. en Geneesk. Congres, Amsterdam, 1915.

9. Bemmelen, J. F. van, Over de phylogenetische beteekenis van het kleurenpatroon der Hepialiden.

Versl. van de Kon. Akad. v. Wetensch., Amsterdam, Deel XXIV, 1916.

10. Botke, J. Bijdruge tot de kennis van de phylogenie der vleugelteeliening bij de Lepidoptera.

Versl. Kon. Akad. van Wetensch., Amsterdam, Deel XXIV, 1916.

11. B ryk, F. Ein Citronenblatt mit einer urspriunglichen Weiszling: zeichnung.

Zool. Anzeiger, Bd. XLIV, 1914. 
12. Chapman, Th. A. On the phylogeny and evolution of the Lepidopterce from a pupal and oval standpoint.

Transact. of the Ent. Soc. of London, 1896.

13. Doegener, P. Die Metamorphose der Inseliten. Leipzig und Beslin, 1909.

14. Dixey, F. A. Wing-markings of Nymphalidae.

Trans. Ent. Soc. of London, Part I, 1890.

15. Dixey, F. A. On the phylogenetic significance of the wing-markings in certain genera of the Nymphalidae.

Transact. Ent. Soc. of London, 1890.

16. Eecke, R. van. A new Hepialid from Sumatra.

Zoöl. Mededeelingen van het Rijksmuseum van Nat. Historie, Leiden, Deel I, 1915.

17. Enderlein, G. Eine einscilige Hemmungsbildung bei Telea polyphemus vom ontogenetischen Standpunkt.

Lool. Jahrbücher, Abt. Anat. und Ontog, der Thiere. Band 16, 1902.

18. Eimer, G. H. Th. Die Artbildung und Verwandtschaft bei den Schmetterlingen. Eine systematische Darstellung der Abänderungen, Abarten und Arten der Segelfalter-ühnlichen Formen der Gattung Papilio. Jena, 1889.

19. Eimer, G. H. Th. Orthogenesis der Schmetterlinge. Ein Beweis bestimmt gerichteter Entwickelung und Ohmmacht der natïrlichen Zuchtwahl bei der Artbildung. Die Entstehung der Arten 1I. Theil.Zugleich eine Lnwiderung an August Weismann. Leipzig, 1897.

20. Göldi, E. Phylogenie der Hexapoden.

dans: L a n g, Handbuch der Horphologie der Wirbellosen Tiere, Jena,1914.

21. Geest, W. Eine Aberration von Rhodocera rhamni und Entwicklung der Pieriden-Fürbung.

Allg. Zeitschr, für Entomologie, Bd. VII, 1902.

22. Gebhard t, F. A. M. W. Die Hauptzüge der Pigmentverleilung im Schmetterlingsflügel im Lichte der Liesegang'schen Niederschläge in Kolloiden.

Verhandl. der Deutsch. Zool. Gesellschaft auf der 228ten Jahresvers. zı Halle, 1912 .

23. Fischer, E. Transmutation der Schmetterlinge infolge Temperaturänderungen. Experimentelle Untersuchungen über die Phylogenese der. Vanessen, Berlin, 1895.

24. Fischer, E. Experimentelle Untersuchungen über die Vererbung erworbener Eigenschaften.

Allg. Zeitschr. für Entom. Bd, VI, 1901.

25. Fischer E. Lepidopterologische Experimental-Forschungen.

Allg. Zeitschr. für Entom., Bd. VII, 1902.

26. Fischer, E. Weitere Untersuchungen über die Vererbung erworbener Eigenschaften.

Allg. Zeitschr. für Entom. Bd, VII, 1902.

27. Hase, E. Untersuchungen über die Mimicry auf Grundlage eines natürlichen Systems der Papilioniden. Bibl. zool. 8 Hft, 1891. 
28. II andlirsch, A. Die fossilen lnsekten und die Phylogenie der resculen Formen. Leipzig, 1908.

29. He nneguy, L. F. L.es Insectes. Morphologie, IReproduction, Embryogénie. Palis, 1904.

30. Herrich-Schäffer. Sammlung nener oder wenig beliannter aussereuropaischer. Schmetterlinge. Regensburg, 1850-1858.

31. Jordan, Karl. An Examination of the Classificatory and some other Resulls of Eimer's Researches on Eastern Papilio's: A Review and Reply. Novitates Zoologicae. Vol. V, 1898.

32. Klug. Versich einer systematischen Feststellung der Insectenfamilie: Panorpatae und Auseinandersetzung ihrer Gallungen und Arten.

Abb. der Kön. Akad. der Wissensch. zu Perlin, 1836.

33. Kirly y, W. F. A hand-book to the Order Lepidoptera.

Allen's Naturalist's Library, London, 1897.

34. Kirby, W. F. A Synonymic Catalogue of Lepidoptera Heterocera (Moths). London, 1892.

35. Linden, M. Gräfin von. Untersuchungen über die Entwicklung der Zeichnung des Schmetterlingsflügets in dev l'uppe.

Zeitschr. f. Wiss. Zool., 65. Bd. 1898.

36. L ind en, M. Gräfin von. Neue untersuchungen über die Entwicklung der Schuppen, Farben und Farbenmuster auf den Flïgeln der Schmetterlinge und Mollen.

Biol. Centralb., Bd. XVIII, 1898.

37. Linden, M. Gräfin von. Die Artbildung und Verwandtschaft bei den Schmelterlingen.

Biol. Centralbl. Bd. XVIII, 1897.

38. Linden, 11. Grïin ron. V'ersuche über den Einflusz äuszerer Verhälmisse auf die Gestallung der. Schnellerlinge.

Illustr. Zeitsclur. für Entom, 4. Bd, 1899.

39. Linden, M. Grälin von. Morphologische und physiologische Ursachen der Flügetzeichnung und Färbung der Insehten mit besonderer Berïcksichtigung der Schmetlerlinge.

Verh. des 5 Intern. Zool. Congresses zu Berlin, 1901.

40. Linden, M, Gräfin von. Die Flägelzeichnung der Insekten - mit besanderev Berüchsichtigung der Zeichnmy der Lepidopteren - Ihro Entuicklung, ihre Ersachen und ihre Bedeutung für den Verwandtschaftlichen Zusammenhang der Arten.

Biol. Centralbl. Bu. XXI, 1901.

41. Linden, M. Gráfin v on Le dessin des ailes des Lépidoplères. Recherches sur son évolution dans l'ontogenèse et la phylogenise des espèces, son origine et sa valeur systémalique.

Ann. Sc. Nat. Zool. Tome XIV, 1902.

42. Linden, M. Gräfin von. Experimentelle Untersuchung über" die Vererbung erworbener Eigenschaften.

Biol. Centralblatt. Bd. XXI, 1902. 
43. Linden, M. Gräfin von. Die Farben der Schmellerlinge und ihre Ursachen.

Leopoldina, 38. Hft. 1902.

44. Linden, M. Gräfin von. Die gelben und rolen Farbstolfe der Vanessen.

Biol. Centralb]. Bd. XXIII, 1903.

45. Linde n, M. Gräfin von. Die Ergebnisse der experimentellen Lepidopterologie.

Biol. Centralbl. Bd. XXIV, 1904.

46. L ij o n e t, P. Trailé anatomique de la Chenille, qui ronge le bois de Saule. La Haye et Amsterdam, 1762.

47. M a y e,$\Lambda$. G. The development of the wing-scales and their pigment in butterflies and moths.

Bulletin of the Museum of Comp. Zoology at Harvard College, Vol. $\mathrm{XX1X}, 1896$.

48. II a yer, A. G. On the color-patterns of the Moths and Butterflies.

Bulletin of the Mus. of Comp. Zool. at Harvard College, Vol. XXX, 1897.

49. M a y er, A. G. On the development of Color in Moths and Butlerflies.

Biological lectures from the Marine Biological Laboratory of Woods Hall, Boston, 1900.

50. II c. Lachlan. R. Synopsis of the species of Panorpa, occurring in Europe and the adjoining countries; with a Description of a singular new species from Java.

Transact. of the Entom. Society of London, 1809.

51. II erifield, F. The efrects of temperalure in the pupal Stage on the colouring of Pieris napi, Vanessa atalanta, Chrysophanus phloeas and Ephyra punctaria.

Transact. Entom. Society. London, 1893.

52. Me yer e, J. C. H, de, Verslag van de 70ste Zomervergadering der Ned. Entom. Vereeniging. 1915.

53. M e y e re, J. C. H. d e, Verslag van de 49ste Wintervergadering van de Ned. Entom. Vereeniging. 1916.

54. M e y e 1'e, J. C. 11. de, Zur Zeichnung des Insekten-imbesonderen des Dipteren- und Lepidopterenflügcls.

Tijdschrift voor Entomologie, Deel IX, 1916.

55. O u demans, J. T h. De Nederlandsche Inseklen. 's Gravenhage, 1900.

56. 0 udemans, J. Th. Etude sur la position de repos chez les Lépidoplères

Kon. Akad. v. Wetenschappen, Amsterdam, 1903.

57. Pan k a th, O. Das Auge der Raupen und Phryganidenlarven.

Zeitschr, für wissensch, Zool. Bd. XLIX, 1890.

$58 \mathrm{P}$ iepers, M. C. Die Farbenevolution (Hhylogenie der Farben) bei den l'ieriden.

Tijdschrift Ned. Dierk. Vereeniging, (2) Deel V, 1898.

59. Piepers, M. C. The evolution of colour in Lepidoptera.

Notes from thie Leyden Museum, Vol. XXII, 1899. 
60. Poult on, E. B. The external Morphology of the Lepidopterous Fupa: its Relation to that of the other Stages and to the Origin and Hislory of Metamorphosis.

Transact. of the Linn. Society of London, 1891.

61. Petersen, W. Bemerikungen zur Systematik der Schmelterlinge.

Allg. Zeitschr. für Entom. Bd. VII, 1902.

62. Rothschild, W. Some new Cossidae from Quecnsland, bred by Mr. F. P. Dodd.

Novitates zoologicae Vol. X, 1903.

63. Schäffer, C. Beilräge zur Histologie der Insekten.

Zool. Jahırb. Abth. füı Anatomie, Bd. III, 1889.

6.t. Schaus, W. Descriptions of New South-American Moths.

Proceedings U. S. National Museum, Vol. XX1X, 1906.

65. Schröder, Chr. Experimental-Untersuchungen bei den Schmellerlingen und deven Entwicklungszuständen.

Illustr. Wochenschr. für Entomol. Bd. I, 1896.

66. Schröder, Chr. Die Zeichnungs-Variabililät von Abraxas grossulariata $L$, gleichzeitig ein Beitrag zur Deszendenztheorie.

Allg. Zeitschr. für Entom. Bd. VHI, 1903.

67. Schröder, Chr. Kritik der von Herrn Dr. E. Fischer (Zürich) aus seinen Lepidopterologischen Experimentalforschungen gezogenen Schlüsse auf Grund einer neuen Erkläming des Wesens derselben.

Ibidem.

68. Schröder, Chr". Handbuch der Entomologie, Jena, 1912.

69. Seitz, A. Cossidae, dans: $\Lambda$. Seitz, Die Gross-Schmetterlinge der Erde. $\Lambda$ bteilung I. Die G. S. des Falaearktischen Faunengebietes.

70. Spuler, A. Zur Stammesyeschichte der Papilioniden.

Zool. Jahrbücher. Abt. Syst. Geogr. und Biolngie der Thiere, Bd. 6, 1891.

71. Spuler, A. Zur Phylogenie und Ontogenie des Flügelgeäders den Schmetterlinge.

Zeitschr. für Wiss. Zool. Bd. L11I, 1892.

7:. Smolian, Kurt. Ueber die Variabilität des braunen Bärenspinners (Arctia caja $L_{\text {. }}$ ) und die Beziehungen desselben $\approx u$ den ihm nächstverwandten Arctiden, gleichzeitig ein Beitrag zur Deszendenztheorie.

Jenaische Zeitschr. für Naturwissenschaft, Bd. 50, 1913.

73. Standfusz, M. Handbuch der palärtitischen Gross-Schmetterlinge für Forscher und Sammler, Jena 1896.

74. Urech, F. Beobachtungen über die verschiedenen Schuppenfarben und die zeill. Succession itres Auftretens (Farbenfelderung) auf den Puppenflügelchen von $V$. urticae und 10 .

Zool. Anzeiger, Bd. XIV, 1891.

75. Urech, F. Chemisch-analytische Untersuchungen an lebenden Raupen Puppen und Schmellerlingen und an ihren sccreten.

Zool. Anzeiger, Bd. XIII, 1890.

76. Walter, A. Palpas maxillaris Lepidopterom.

Jenaische Zeitschr. für Natur'w. BıI. XVIII, 1884. 
77. Walter, A. Beiträge zur Morphologie der Schmetterlinge. I Mundteile. Jenaische \%eitschr. für Naturw. Bd. XVIII, 1885.

78. Weismann, A. Studicn zur Deszendenztheorie. I. Ueber den Saisondimorphismus der schmelterlinge. Leipzig, 1875.

79. Westwood, J. O. Nonograph of the Genus Panorpa with Descriptions of some species belonging to other allied Genera.

Transact. of the Entom. Soc, of London, Vol. IV, 1845-1847.

80. Woodworth, C. W. The wing veins of Insecls.

Contributions from the Zoological Laboratory of the Museum of Comp. Zool, at Harvard-College, Univ, of California. Publications.

Technical Bulletins. Entomology, Vol. 1, 1906. 


\section{LEGENDE DES PIANCHES.}

\section{PLANCHE I.}

Fig. 1, Cossus cossus, dessus, (Coll. De Boer et $\mathrm{K}$ oo $\mathrm{y}$ ).

Fig. 2, " ", dessous, ( D B Boer et Kooy).

Fig. 3, " ", dessus, ( "Kallenbach).

Fig. 4, " , dessous, ( Kallenbach).

Fig. 5, " ", dessus, (" Oudemans).

Fig. 6, " ". dessous, ( " Oudemans).

Fig. 7, " ", dessus, ( $~ 0$ Oudemans).

Fig. 8, Cossus palmaris, dessus, (Coll. Staudinger).

Fig. 9, Cossus sp., dessus, (Coll. (Staudinger).

Fig. 10, Cossus sp. (Equateur), dessus, (Coll. Staud inger).

\section{PLANCHE II.}

Fir. 1, Zeuzera pyrina, dessus, (Coll. personelle).

Fig. 2,, sp., (Afrique), dessus, (Coll. Jard. Zool.).

Fig. 3, Tyleutes (d'urvillei?) つ’, dessus, (Coll. Jard. Zool.).

Fig. 4 " " " dessous, ( " ").

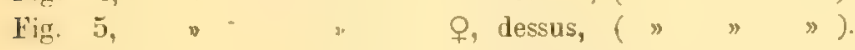

Fig. 6, " " $"$ dessous, ( " ").

Fig. 7, " sp., dessus, (Coll. Jard, Zool.).

Fig. 8, " " dessous, (" " ").

Fig. 9, " sp., dessus, (" " ").

Fir. 10, " " dessous, (" ").

Fig. 11, Endoxyla lituratis, dessus, (Coll. Jard. Zool.).

Fig. 12, " strix, dessus, (Coll. Staudinger).

\section{PLANCHE III.}

Fig. 1, Endoxyla strix, dessus, (Coll. Staudinger).

Fig. 2, " ", dessous, ( "

Fir. 3, " 3, , dessus, (Coll. Jard. Zool.).

Fig. 4, " , ㅇ, dessous, (" " ) .

Fig. 5, " ligneus, o", dessus, (Coll. Jard. Zool.).

Fig. 6, Langsiorfia franliii, dessus, (Coll. Jari. Zool,). 
Fig. 7, Prionoxystus robiniae, 07, dessus (Coll. Jard. Zool.).

Fig. 8, " " dessous ( " " $"$ ).

Fig. 9, Arbela sp, dessus, (Coll, Jard. Zoöl.).

Fig. 10, " " dessous, ( " " ).

Fig. 11, Eriocrania sparmannella, dessus, (Coll. Musée de Leyde).

Fig. 12, Calpe capucina, dessus, (Coll. Kallenbach).

\section{PLANCHE IV.}

Fig. 1, Phryganea varia, dessus, (Coll. personelle).

Fig. 2, " grandis, dessus, (Coll. De Meyere).

Fig. 3, Limnophilus affinis, dessus, (Coll. personelle).

Fig. 4, Neuronia imperialis, dessus, (Coll. Musée de Leyde).

Fig. 5, " " var. regina, dessus, (Coll. Musée de Leyde).

Fig. 6, " reticulala, dessus, (Coll. Musée de Leyde). 


\section{FIGURES DANS IE TEXTE.}

Fir 1. Quelques écailles de Cossus palmaris entre An et $A n_{\text {. }}$.

Fig. 2. Cellule $R_{3}-R_{4}$ de Cossus palmaris. Dessus de l'aile antérieure gauche ... . . . . . . . . . 42

Fig. 3. Une des taches foncées de Zeuzera pyrinc . . . . . . . 51

Fig. 4. Une des taches pâles de Zeuzera pyrina . . . . . . . 51

Fig. 5. Fragment de l'aile antérieure de Tyleutes sp. (d'urvillei?) f.

Transitions de traits en réseau coloré. . . . . . . $50^{\circ}$

Fig. 6. Fragment de l'aile antérieure de Iyleutes sp. (d'urvillei?) \&. . it

Fig. 7. Cellule $\mathbf{I I}_{2}-\mathbf{M}_{3}$ de l'aile antérieure de Iyleutes sp. Dessus . . 57

Fig. 8. Cellule costale $(\mathrm{C}-\mathrm{SC})$ de l'aile postérieure droite de Xyleules strix ㅇ․ . . . . . . . . . . . . . . 67

Fig. 9. Cellule costale (C-SC) de l'aile postérieure gauche de $\mathrm{X} y$ leutes strix ㅇ. . . . . . . . . . . . . . 67

Fig. 10. Cellule $\mathbf{M}_{3}-\mathrm{Cu}_{1}$ de l'aile antérieure de Prionoxystus robiniae. Dessus . . . . . . . . . . . . . 75

Fig. 11. Cellule du dessous de l'aile postérieure de Vanessa io avec les traits effilochés . . . . . . . . . . . . 94

Fig. 12. Aile de Bittacus corethrarius Ramb. . . . . . . . . . 123 


\section{TABLE DES MATIERES.}

Chapitre I. Introduction . . . . . . . . . . . . . . 1

A. Historique. . . . . . . . . . . . . 1

1. Les recherches ontogénétiques . . . . . . . . . . 1

2. Les recherches morphologiques comparées . . . . . . 11

3. Les recherches expérimentales . . . . . . . . . . 18

4. Aberrations et anomalies. . . . . . . . . . . . 21

5. Les couleurs et leurs pigments. . . . . . . . . . . 22

6. Rapport entre le dessin des Lépidoptères et d'autres ordres d'Insectes . . . . . . . . . . . . . 25

B. Les opinions d'aujourd'hui sur le dessin primitif . . . . . . 29

Chapitre II. Descriptions des diver's dessins. . . . . . . . . . . 32

A. Matériel et méthode . . . . . . . . . . . . . . . 32

B. La terminologie de l'aile . . . . . . . . . . . . . . 33

C. La famille des Cossides . . . . . . . . . . . . . 35

1. Cossus cossus I.. . . . . . . . . . . . . . . . 36

2. Cossus palmaris . . . . . . . . . . . . . 40

3. Cossus sp.. . . . . . . . . . . . . . . . . . 44

4. Cossus sp... . . . . . . . . . . . . . . . 46

5. Cossus terebra T. . . . . . . . . . . . . . 48

6. Cossus modestus Stgr. . . . . . . . . . . . . 48

7. Zeuzera pyrina L. . . . . . . . . . . . . . 49

8. Zeuzera sp. . . . . . . . . . . . . . . . 51

9. Duomites leuconotus Walker. . . . . . . . . . . 52

10. Xyleutes pyracmon Cram. o . . . . . . . . . . 54

11. Xyleutes (d'urvillei ?). 우 . . . . . . . . . . . 55

12. Xyleutes sp. (d'urvillei ?) ठ․ . . . . . . . , , . 59

13. Xyleutes sp. . . . . . . . . . . . . . . . 59

14. Xyleutes sp. . . . . . . . . . . . . . 62

15. Xyleutes lituratis Donov. . . . . . . . . . . . 64

16. Xyleutes (Endoxyla) strix L.. ㅇ.. . . . . . . . . 65

17. " " $" \delta^{7}$. . . . . . . . 68

18. Endoxyla ligneus Butl. . . . . . . . . . . 70

19. Langsdorfia frankii Hb. . . . . . . . . . . . . . 71

20. Prionoxystus robiniae Boisd. . . . . . . . . . . . 73 
21. Hypopta thrips Hb. . . . . . . . . . . . 75

22. Holcocerus arenicola Stgr. . . . . . . . . . 76

23. Phragmatoecia castanea Hb. . . . . . . . . . . 77

24. Stygia ledereri Stgr. . . . . . . . . . . . . . 77

25. Stygia australis Lr. . . . . . . . . . . . 78

D. La famille des Arbélides. . . . . . . . . . . . . . . 79

1. Arbela sp. . . . . . . . . . . . . . . . . 79

E. La famille des Microptérygides . . . . . . . . . . . . 81

1. Eriocrania sparmannella F. . . . . . . . . . . . . 81

F. L'ordre des Trichoptères . . . . . . . . . . . . . . . 82

1. Phryganea varia F. . . . . . . . . . . . . . . 82

2. Phryganea grandis L.. . . . . . . . . . . . . . 84

3. Limnophilus marmoratus Curt. . . . . . . . . . . 8\%

4. Limnophilus flavicornis F. . . . . . . . . . . . 85

5. Limnophilus rhombicus L. . . . . . . . . . . . . 86

6. Limophilus affinis Curt. . . . . . . . . . . . 86

7. Neuronia imperialis Snell. v. Voll. . . . . . . . . . 86 ;

8. Neuronia imperialis, vas, regina Lachl. . . . . . . ST

9. Neuronia reticulata L. . . . . . . . . . . . 8!

Chapitre III, Les divers motifs. . . . . . . . . . . . . . . 94)

Chapitre IV. Le dessin primitif et ses modifications. . . . . . . . 10n

Chapitre V. L'origine du dessin primitif. . . . . . . . . . . . 11fi

Chapitre V1. Résumé . . . . . . . . . . . . . . . . . 131

Bibliographie . . . . . . . . . . . . . . . . . . . . 137

Légende des planches . . . . . . . . . . . . . . . . . . 14:.

Figures dans le texte. . . . . . . . . . . . . . . . . . 14i 

$=$ 


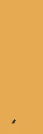

BOEKURUKLHRU TOORUEEN L. J. ERILL, IEIDEN. 


Planche I.
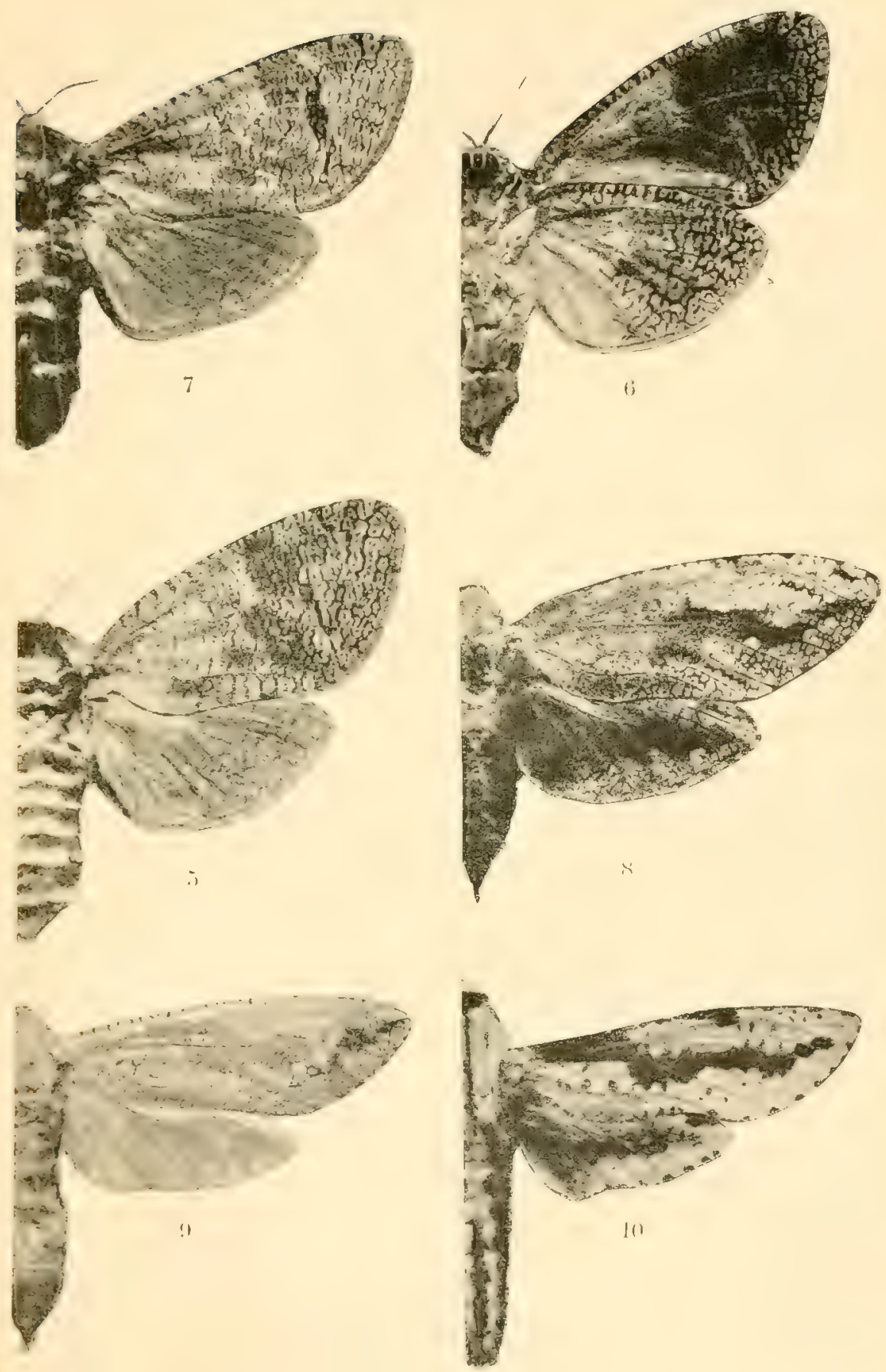

J. Bотке. Les motifs primitifs etc.
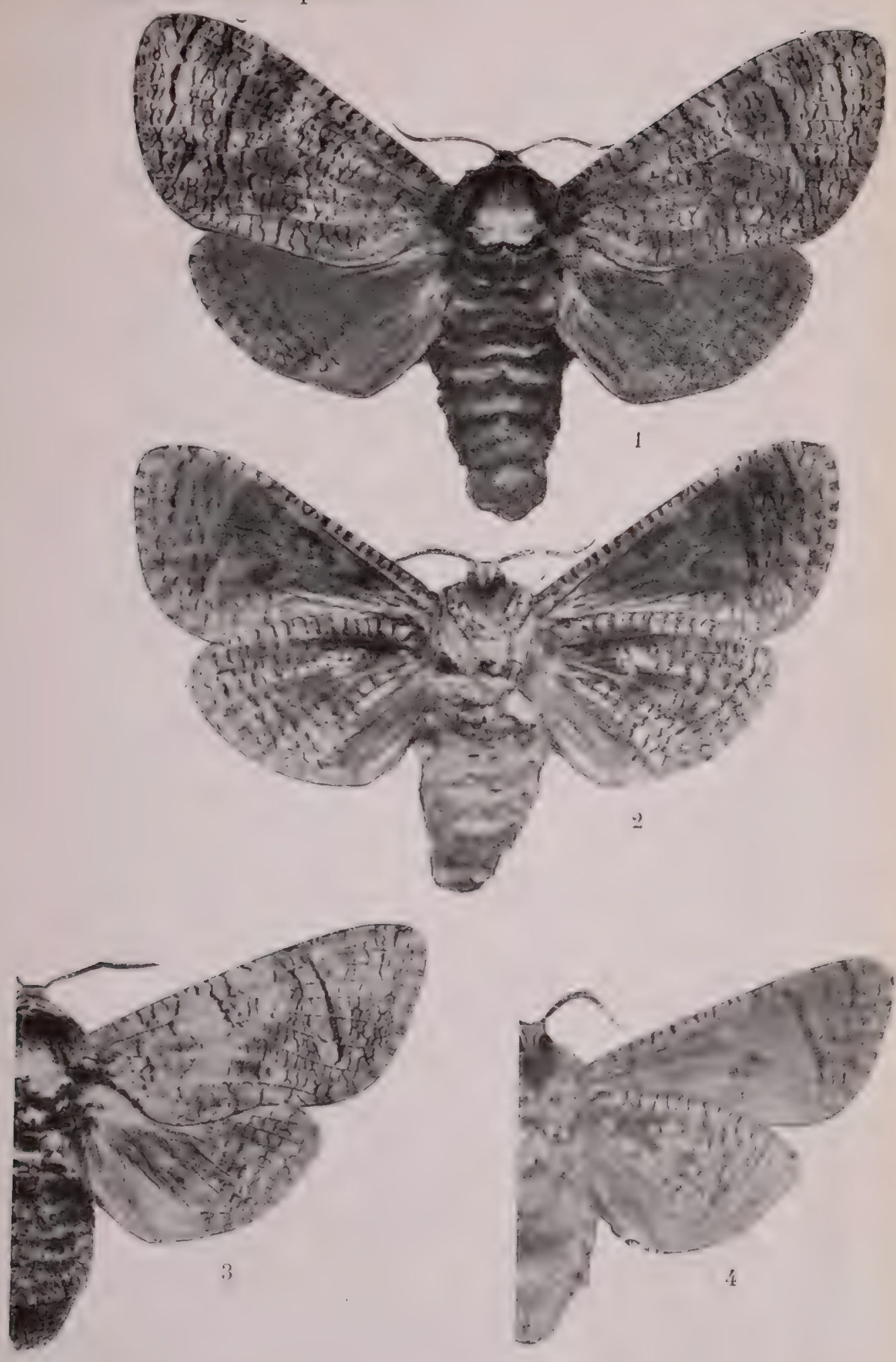

Vix Egnoxd phot.

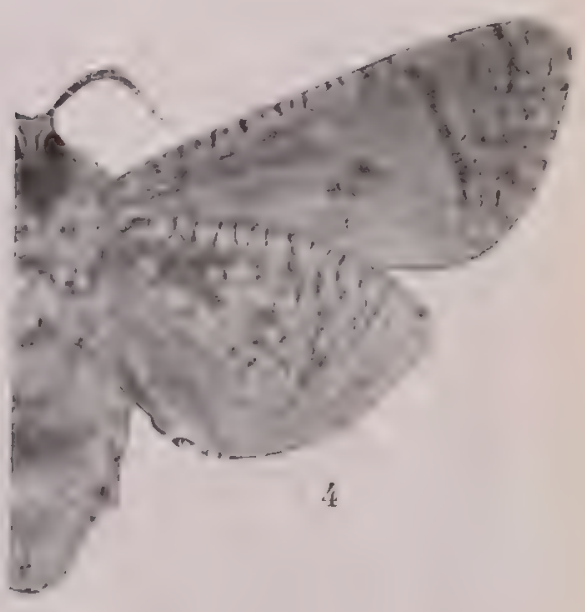

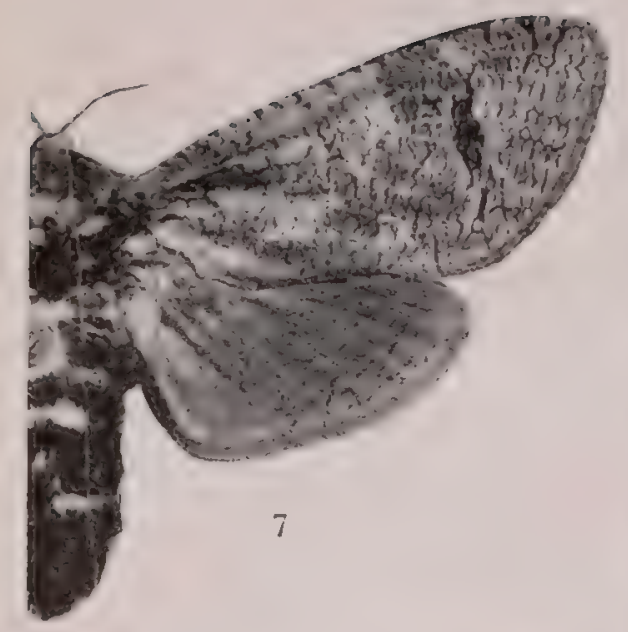
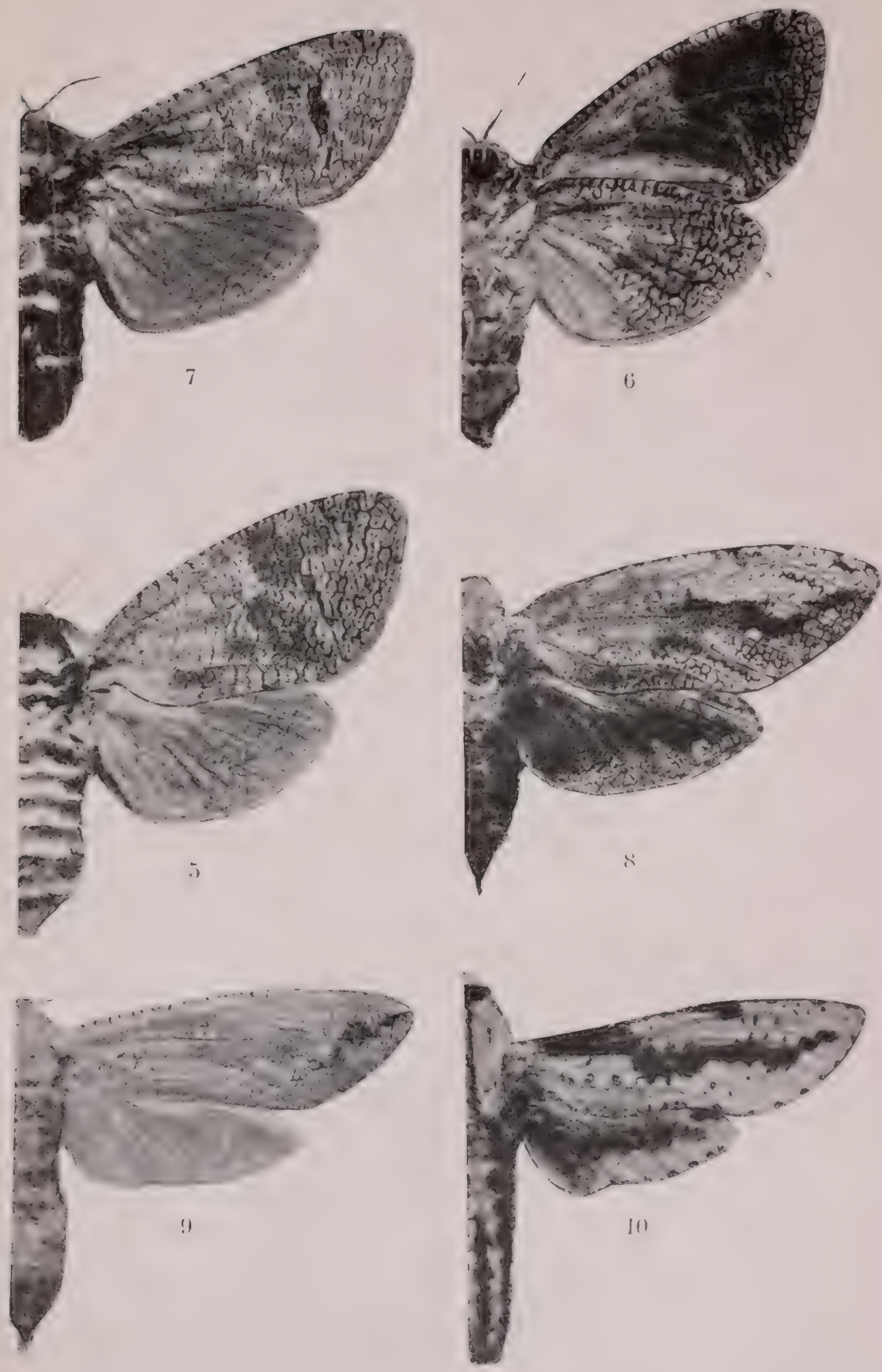

Planche II.
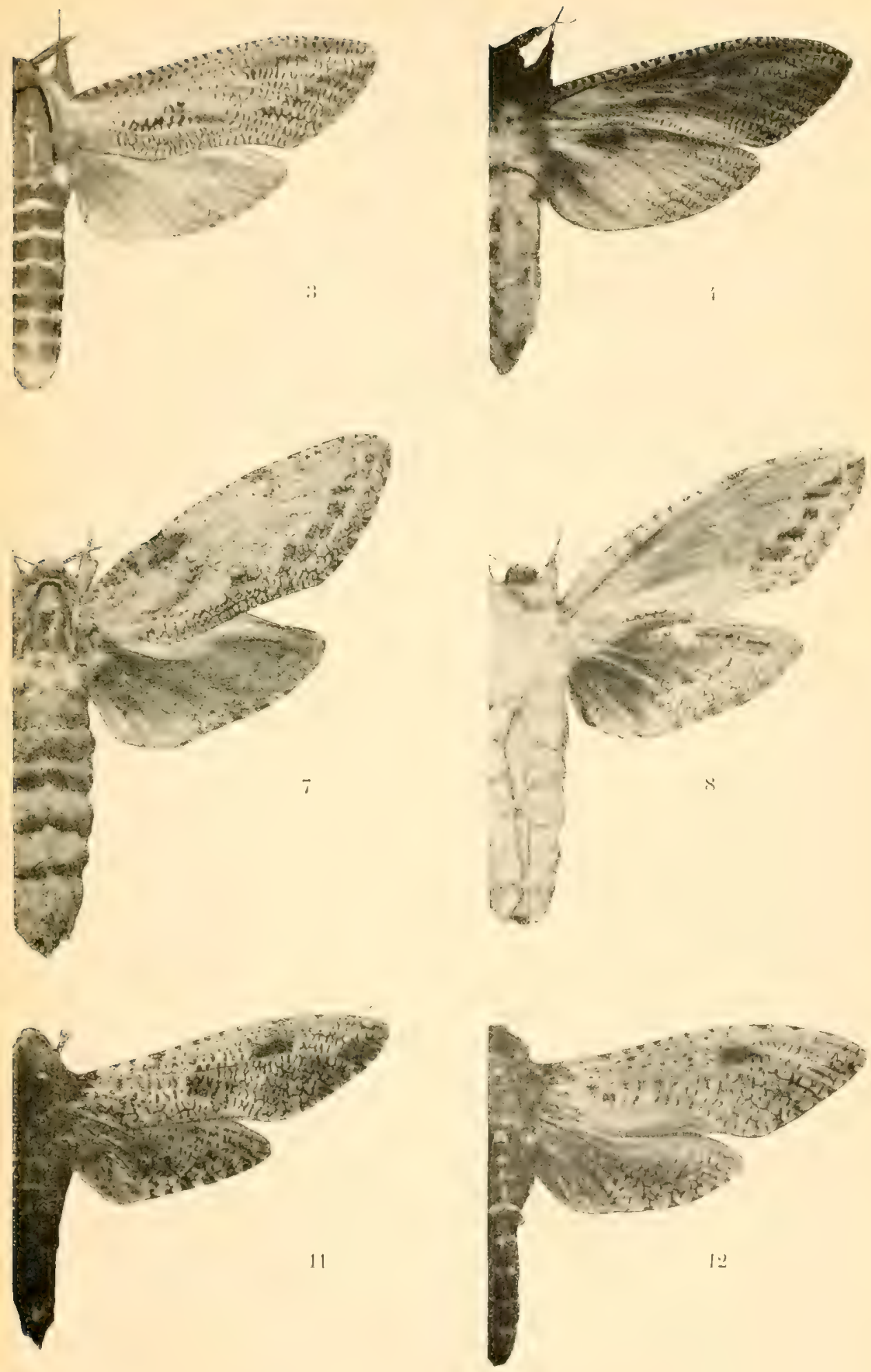

J. BоTKE. Les motifs primitifs etc.
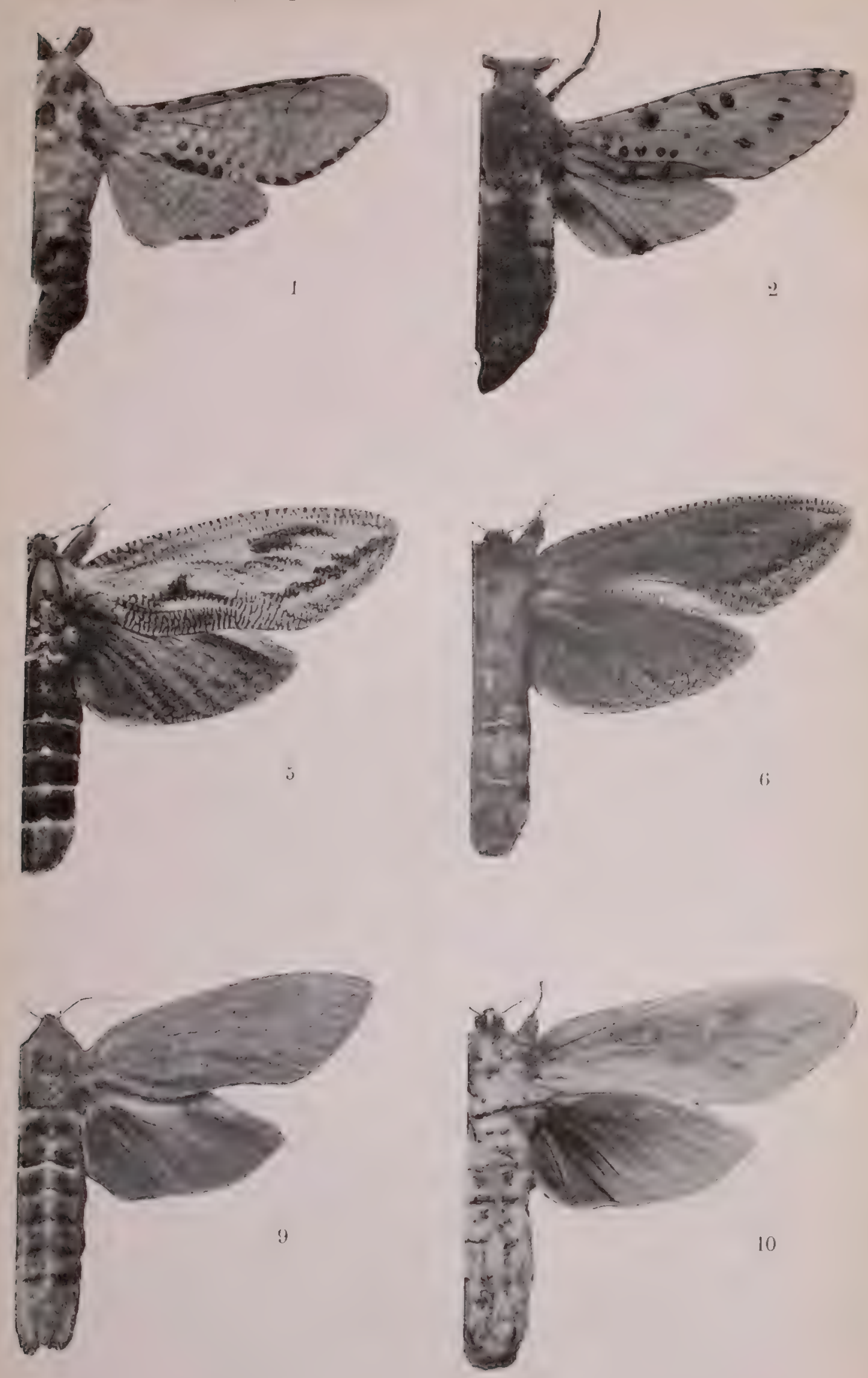
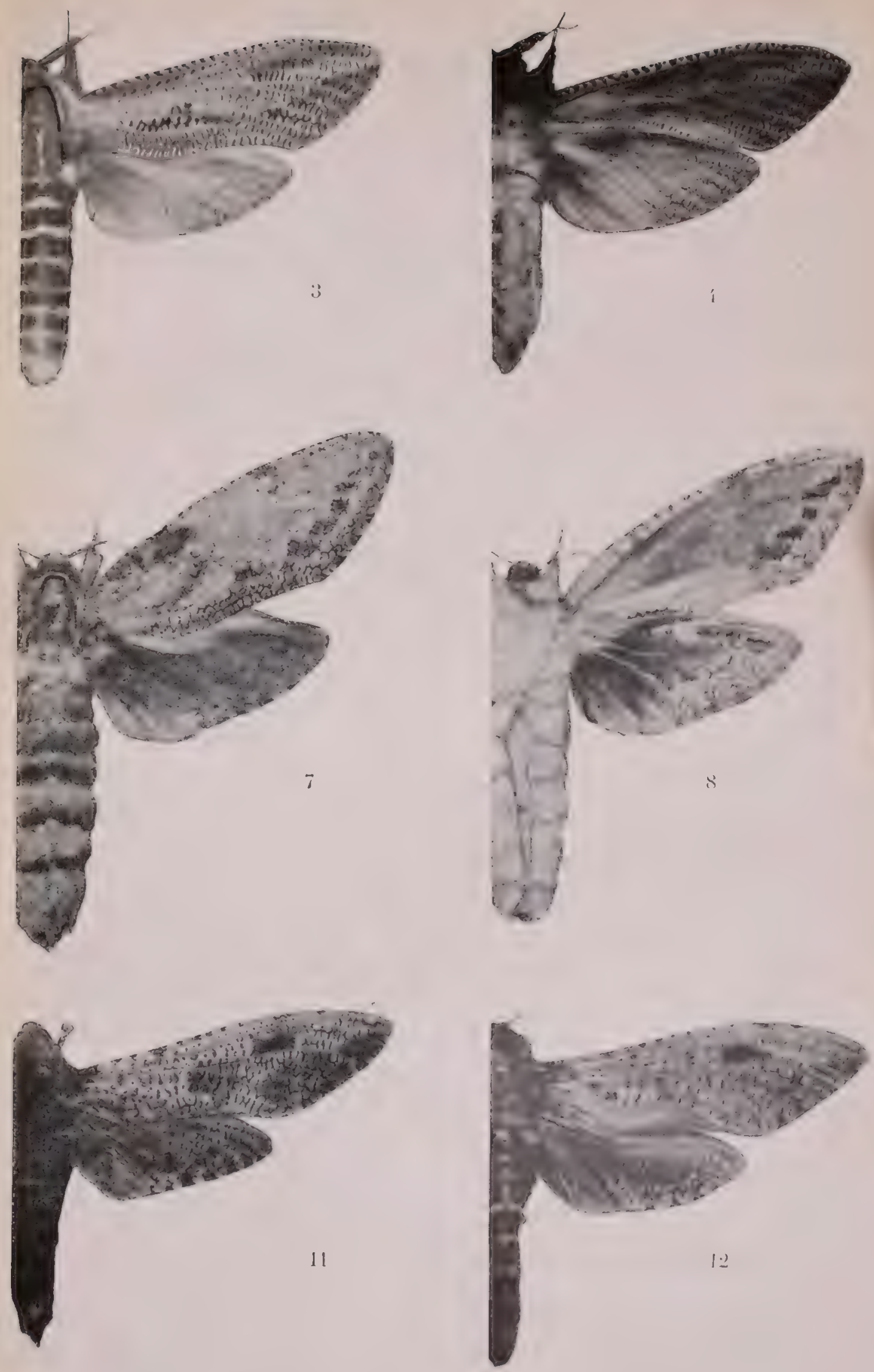


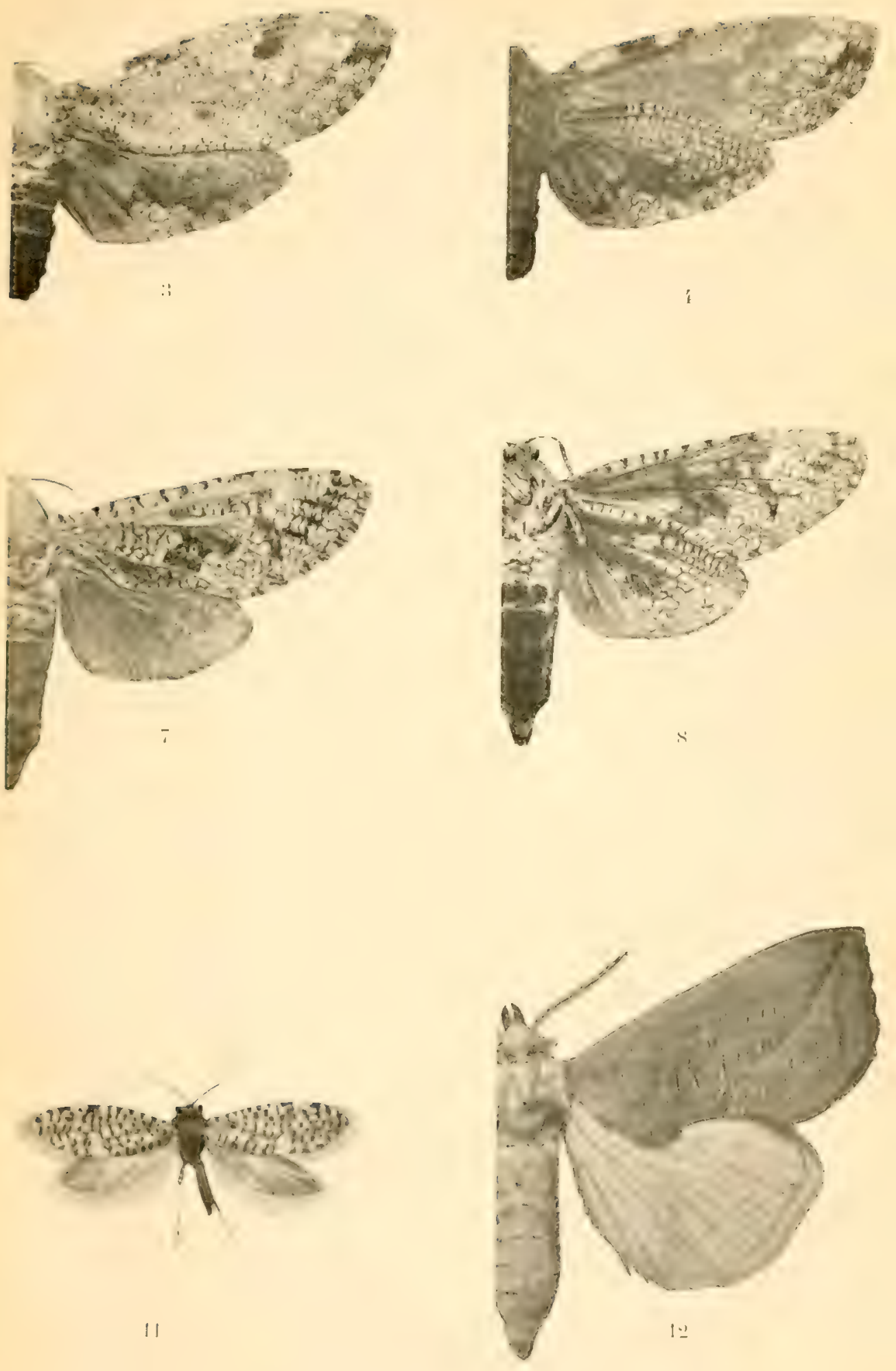




J. Bотке. Les motifs primitifs ete.

Planche IV.
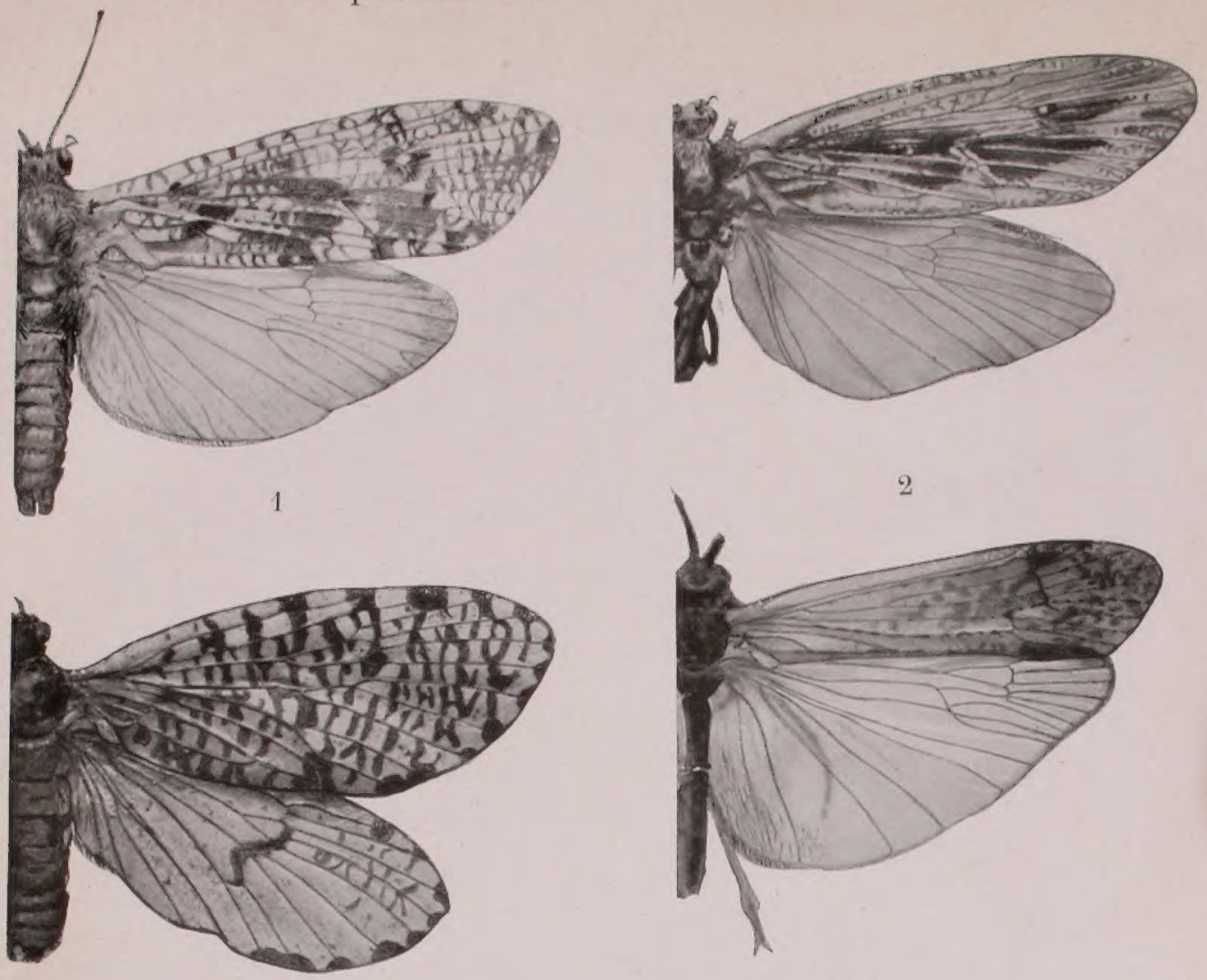

6
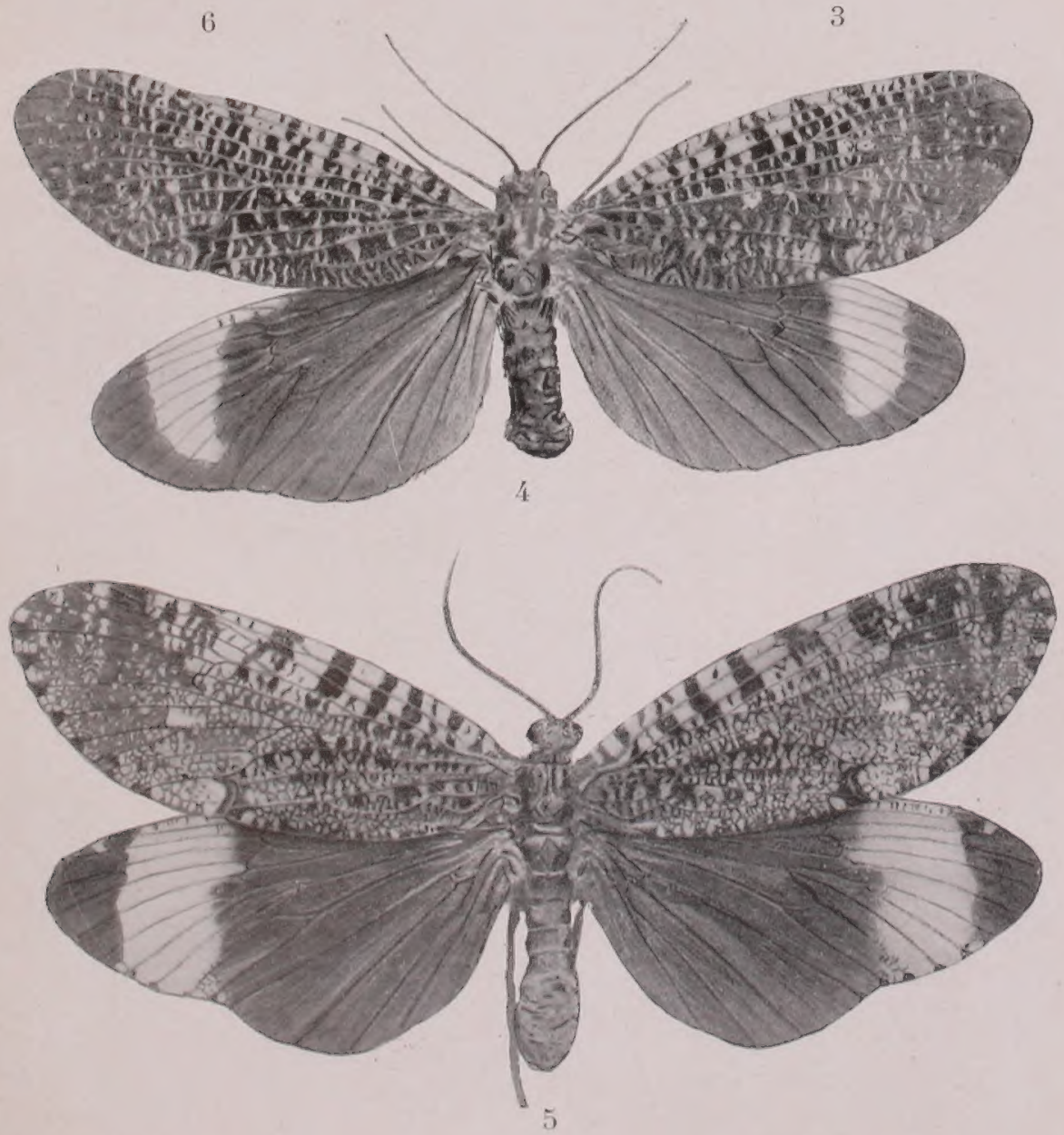

Var Egmond phot. 

$\checkmark$ 
IIU UIUIUUUMAN INSITUTION LIBRARIES

39088000842245 